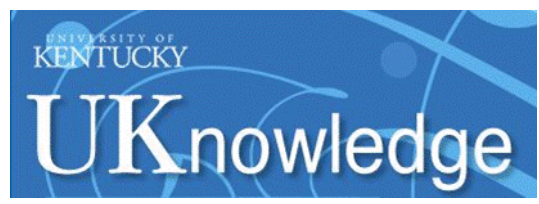

University of Kentucky

UKnowledge

\title{
PATTERN EVALUATION FOR IN-PLANE DISPLACEMENT MEASUREMENT OF THIN FILMS
}

Phanikrishna Thota

University of Kentucky, phani@engr.uky.edu

Right click to open a feedback form in a new tab to let us know how this document benefits you.

\section{Recommended Citation}

Thota, Phanikrishna, "PATTERN EVALUATION FOR IN-PLANE DISPLACEMENT MEASUREMENT OF THIN FILMS" (2003). University of Kentucky Master's Theses. 307.

https://uknowledge.uky.edu/gradschool_theses/307

This Thesis is brought to you for free and open access by the Graduate School at UKnowledge. It has been accepted for inclusion in University of Kentucky Master's Theses by an authorized administrator of UKnowledge. For more information, please contact UKnowledge@lsv.uky.edu. 


\section{ABSTRACT OF THESIS}

\section{PATTERN EVALUATION FOR IN-PLANE DISPLACEMENT MEASUREMENT OF THIN FILMS}

The term "Gossamer" is used to describe ultra-lightweight spacecraft structures that solve the aerospace challenge of obtaining maximum performance while reducing the launch costs of the spacecraft. Gossamer structures are extremely compliant, which complicates control design and ground testing in full scale. One approach is to design and construct smaller test articles and verify their computational models experimentally, so that similar computational models can be used to predict the dynamic performance of full-scale structures. Though measurement of both in-plane and out-of-plane displacements is required to characterize the dynamic response of the surface of these structures, this thesis lays the groundwork for dynamic measurement of the inplane component. The measurement of thin films must be performed using non-contacting sensors because any contacting sensor would change the dynamics of the structure. Moreover, the thin films dealt with in this work are coated with either gold or aluminum for special applications making the film optically smooth and therefore requiring a surface pattern. A Krypton Fluoride excimer laser system was selected to fabricate patterns on thin-film mirror test articles. Parameters required for pattern fabrication were investigated. Effects of the pattern on the thin-film dynamics were studied using finite element analysis. Photogrammetry was used to study the static in-plane displacement of the thin-film mirror. This was performed to determine the feasibility of the photogrammetric approach for future dynamic tests. It was concluded that photogrammetry could be used efficiently to quantify dynamic in-plane displacement with highresolution cameras and sub-pixel target marking.

KEYWORDS: Gossamer spacecraft, in-plane displacement, laser ablation, photogrammetry, finite element analysis.

Phanikrishna Thota

July 24, 2003

Copyright (C) Phanikrishna Thota 2003 


\title{
PATTERN EVALUATION FOR IN-PLANE DISPLACEMENT MEASUREMENT OF THIN FILMS
}

\author{
By
}

\section{Phanikrishna Thota}

\begin{tabular}{c} 
Dr. Suzanne Weaver Smith \\
\hline Director of Thesis \\
Dr. George Huang \\
\hline Director of Graduate Studies \\
July 24,2003 \\
Date
\end{tabular}




\section{RULES FOR THE USE OF THESES}

Unpublished theses submitted for the Master's degree and deposited in the University of Kentucky Library are as a rule open for inspection, but are to be used only with due regards of the rights of the authors. Bibliographical references may be noted, but with quotations or summaries of parts may be published only with the permission of the author, and with the usual scholarly acknowledgements.

Extensive copying or publication of the thesis in whole or in part also requires the consent of the Dean of the Graduate School of the University of Kentucky. 


\section{THESIS}

\section{Phanikrishna Thota}

\section{The Graduate School}

\section{University of Kentucky}

2003 


\title{
PATTERN EVALUATION FOR IN-PLANE DISPLACEMENT MEASUREMENT OF THIN FILMS
}

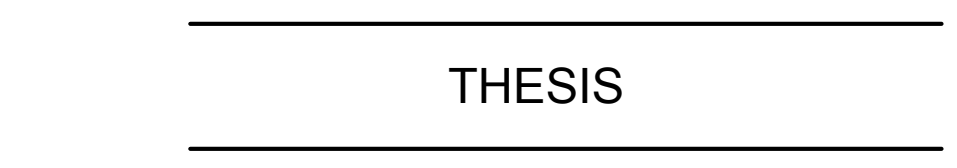

A thesis submitted in partial fulfillment of the requirements of the degree of Master of Science in the College of Engineering at the University of Kentucky

By

\author{
Phanikrishna Thota \\ Lexington, Kentucky
}

Director: Dr. Suzanne Weaver Smith, Associate Processor of Mechanical Engineering

Lexington, Kentucky

2003

Copyright (C) Phanikrishna Thota 2003 


\section{Dedication}

To my mother for her relentless belief in me that brought me to this stage 


\section{ACKNOWLEDGEMENTS}

The following thesis, while an individual work, benefited from the insights and direction of several individuals. Dr. Suzanne Weaver Smith, my Thesis Chair, has been role model for most of my graduate tenure at the University of Kentucky. She exemplifies the hard work and dedication, which I strive to emulate. I would like to thank my co-advisor, Dr. Jack Leifer for his time and insight, which reflected in overall quality of this work. I also convey my sincere appreciation to Dr. Janet K. Lumpp for her guidance in the fabrication process using excimer laser. I am obliged to Dr. Keith Rouch for being a member of my graduate committee and guiding me in finite element analysis of thin-film mirrors.

I am greatly indebted to Kentucky Science and Engineering Foundation (KSEF) for providing financial support for this work. I would like to take the opportunity to extend my gratitude to Dr. Jamey Jacob who helped me with optical equipment and cameras for the experimental setup. I owe special gratitude to Mr. Rodney Bradford, President, United Applied Technologies, for providing thin films for this work.

My friends Rahul, Naveen, Aditi and Rajeswari were very supportive during my two-year stay in Lexington. I would like to thank them for their affection and love that made my stay memorable. My heartiest thanks to all the members of Dynamic Structures and Controls lab for their support all through this course of research. Prem helped me in writing this thesis.

I feel most indebted to my mother, who always taught me the importance of education in life. She taught me that patience and perseverance are the two tools that can take a human being to heights of success. Her words always encouraged me to face the unpredictable hardships and pushed me forward with her love when my abilities faltered. I would like to take this opportunity to thank her for molding me into a successful being and dedicate this thesis to her. My father and brother stood by me whenever I needed them the most. This appreciation would be incomplete without mentioning about my little dog "Micky", though not physically present it is always in my thoughts as my best friend.

Phanikrishna Thota 


\section{TABLE OF CONTENTS}

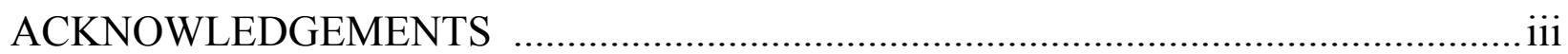

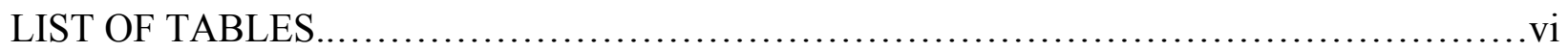

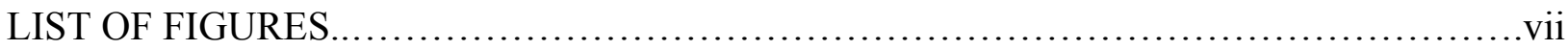

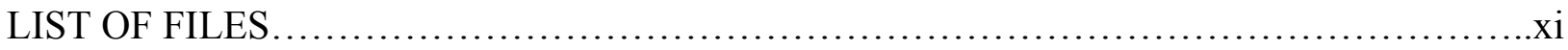

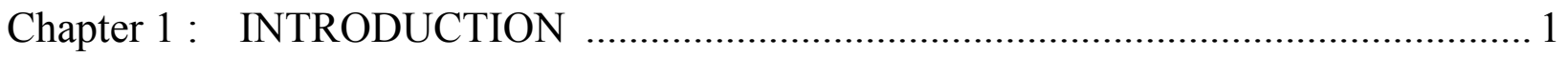

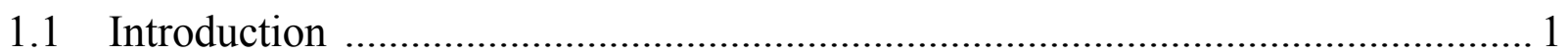

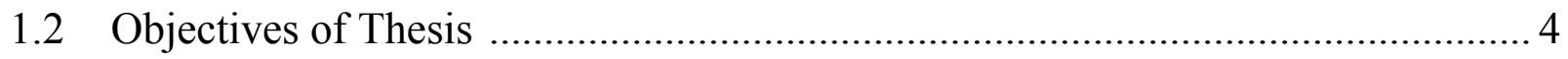

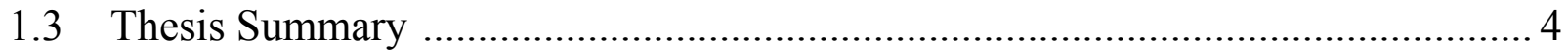

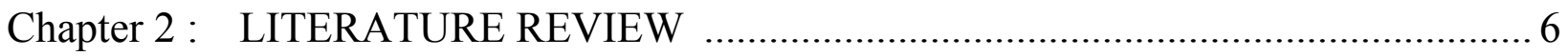

2.1 Partial History of Ultra-light-weight Space Structures ......................................... 6

2.2 Techniques for Measuring In-plane Displacements ............................................... 7

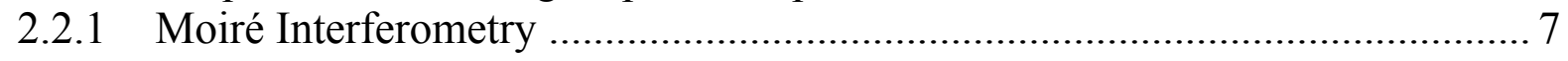

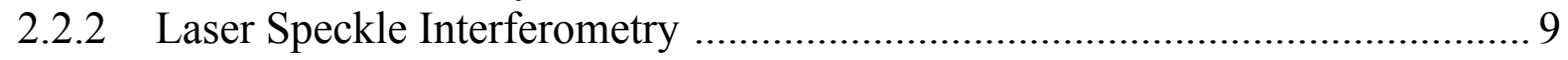

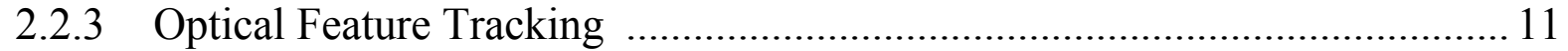

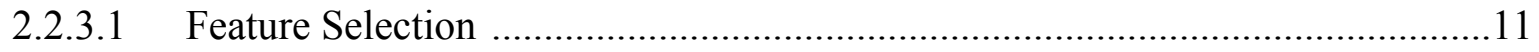

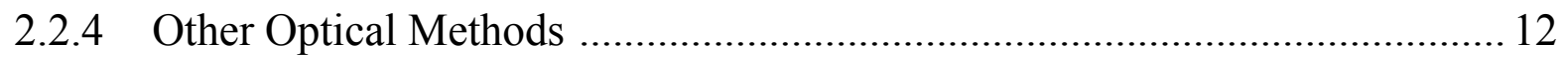

2.3 Review of Thin-Film Dynamics and Measurement …….................................. 13

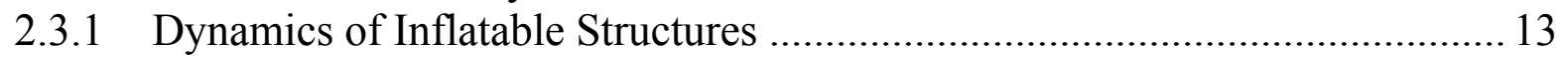

2.3.2 Static Measurement of Thin-film Surfaces .................................................. 14

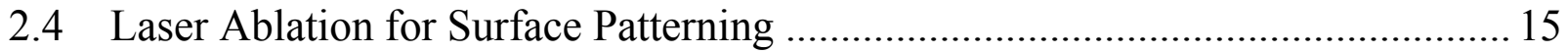

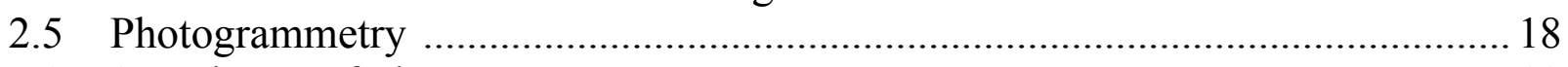

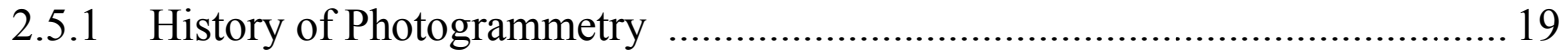

Chapter 3 : TEST ARTICLE FABRICATION PROCESS: MOUNTING AND

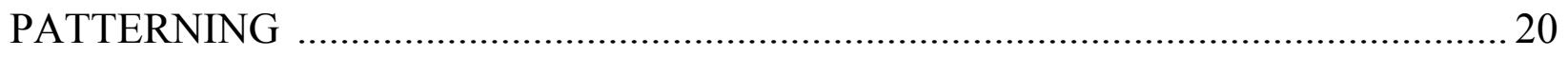

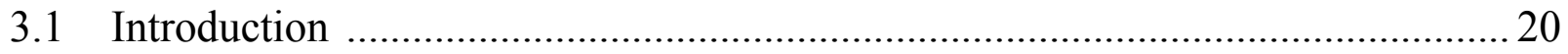

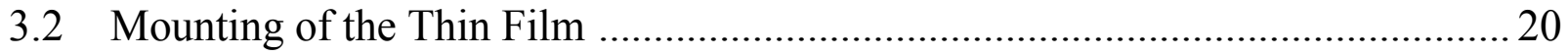

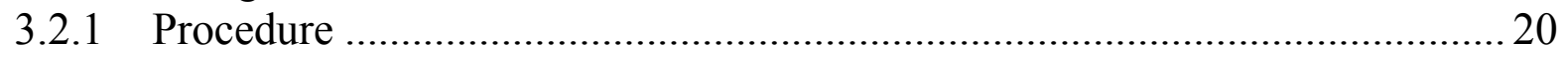

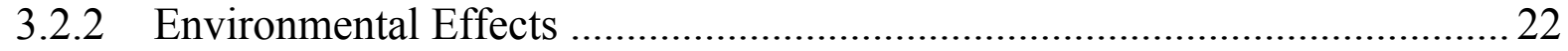

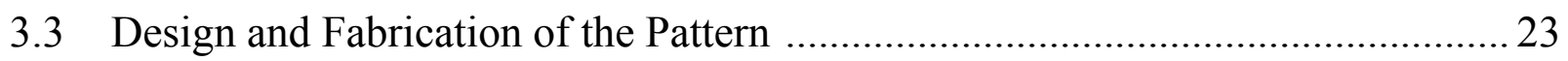

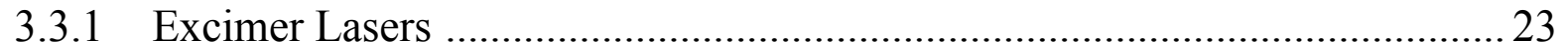

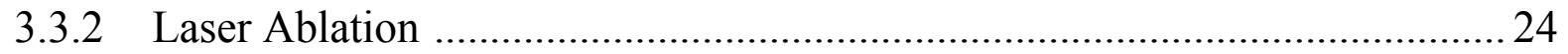

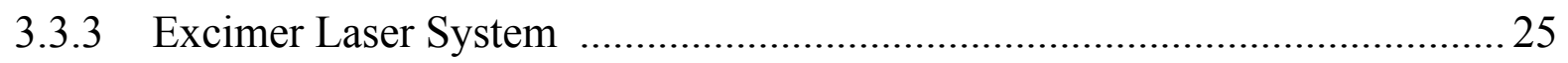

3.3.3.1 Laser Generation System …………………….....................................26

3.3.3.2 Optical Delivery System ……………......................................................26

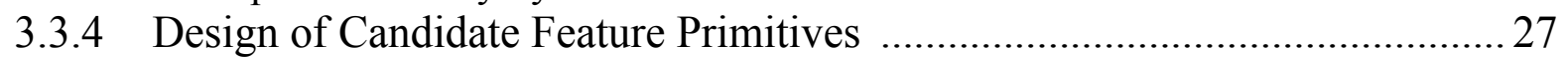


Chapter 4: FINITE ELEMENT ANALYSIS OF ETCHED THIN-FILM MIRRORS. 35

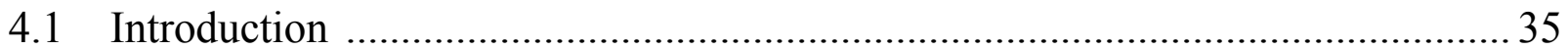

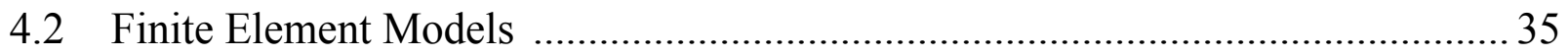

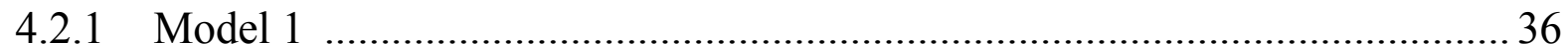

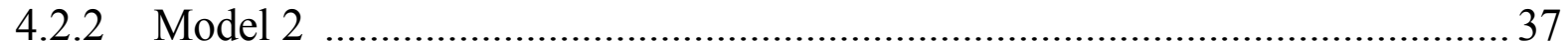

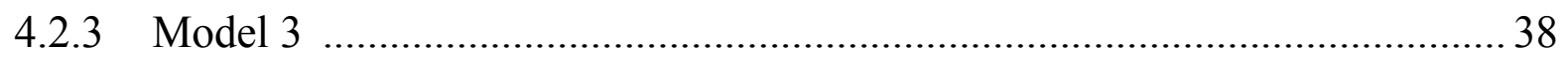

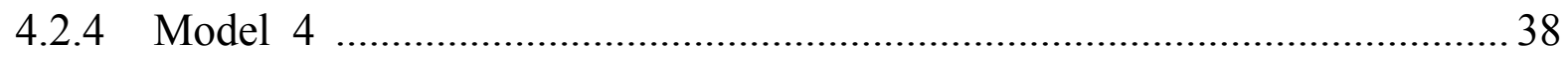

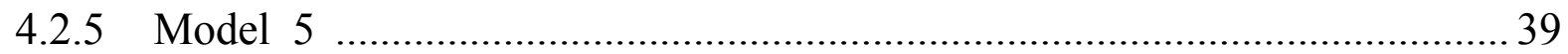

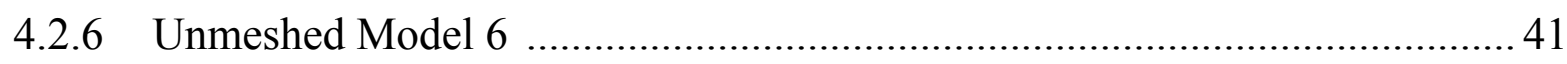

4.3 Modal Analysis of the Finite Element Models ……........................................... 42

4.3.1 Modal Analysis of the Test Article Finite Element Models ......................... 43

4.3.2 Modal Analysis of the $0.5 \mathrm{~m}$ Diameter Thin-film ........................................ 44

4.4 Static Analysis of the Test Article Finite Element Models ................................... 45

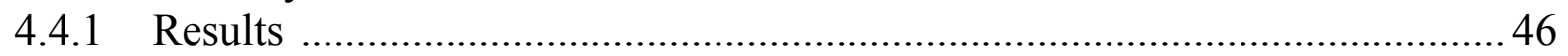

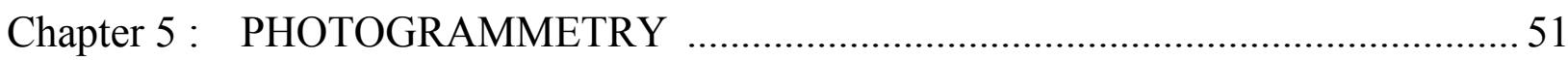

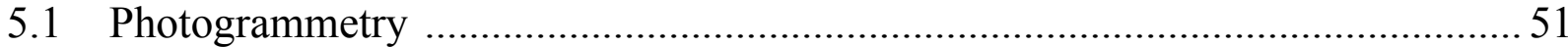

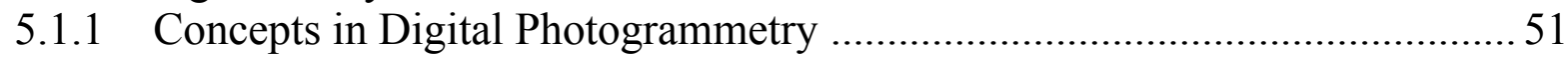

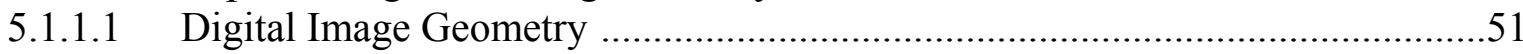

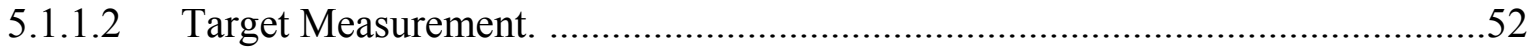

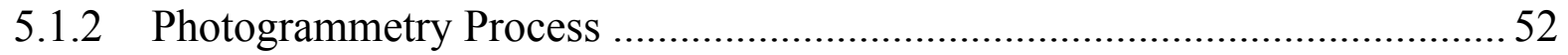

5.2 Photogrammetry of Patterned Thin-Film Mirrors ............................................... 59

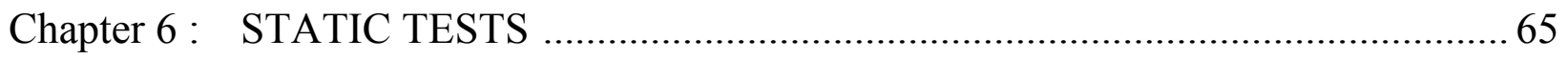

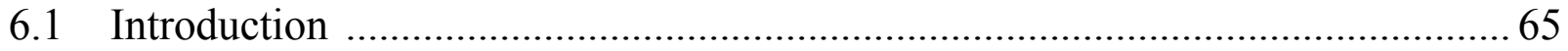

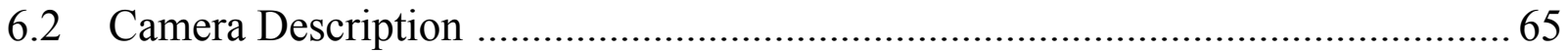

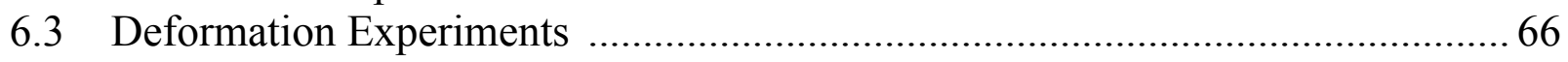

6.3.1 Results of the Deformation Experiments ……............................................ 70

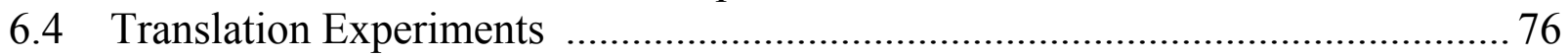

6.4.1 Results of the Translation Experiments …….............................................. 79

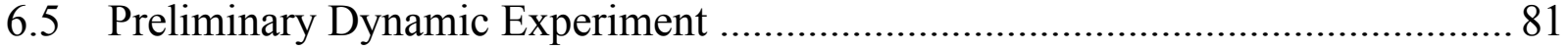

Chapter 7 : SUMMARY, CONCLUSIONS AND FUTURE WORK …………........... 83

7.1 Introduction 83

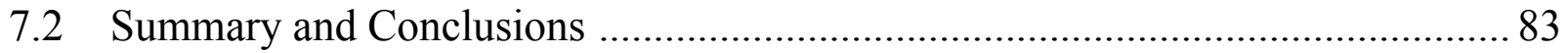

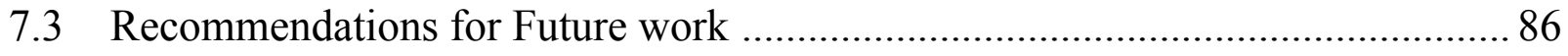

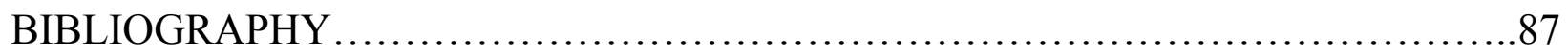

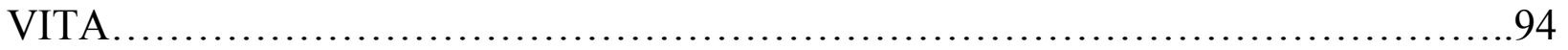




\section{LIST OF TABLES}

Table 3-1: Various excimer lasers and their corresponding wavelengths ............................... 24

Table 3-2: Candidate feature primitives incorporated into the pattern fabricated on the thin-film

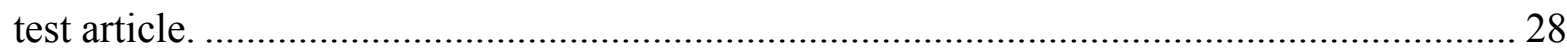

Table 3-3: Laser parameters chosen for pattern fabrication ................................................. 32

Table 4-1: Material Properties used in the Finite Element Models .......................................... 36

Table 4-2: Fundamental Frequencies of Different Models ................................................. 44

Table 4-3: FE results summarizing maximum displacement components of the thin-film mirror50

Table 4-4: Comparison of the results for variation in the thickness of the polymer. ................ 50

Table 6-1: Fabricated and measured line lengths for the fourteen lines at $0 \mathrm{~N}$ load .................. 71 


\section{LIST OF FIGURES}

Figure 1-1: Model of a solar sail ...................................................................................... 2

Figure 1-2: Model of a solar array in Lockheed Martin . ........................................................ 3

Figure 1-3: Crucial technologies for the development of ultra-lightweight structures................ 3

Figure 2-1: Schematic representation of the optical system required for Moiré interferometry.... 8

Figure 2-2: Moiré Interferometry fringe pattern about a fatigue crack. ............................... 8

Figure 2-3: Speckles on both the undeformed membrane and outside of the microphone.......... 10

Figure 2-4: The interferogram of the steel membrane as it bends from its equilibrium position

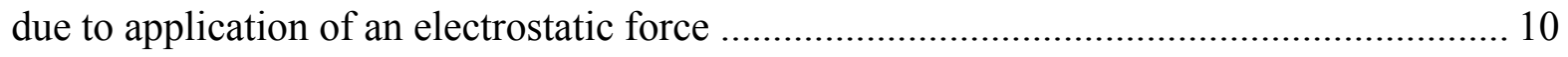

Figure 2-5: Schematic of Stroboscopic Interferometer System ......................................... 13

Figure 2-6: Groove ablated on gold thin film by femtosecond laser pulse of $70 \mathrm{~nJ}$ energy (left); Cross-section of the groove cut by laser pulse of 70nJ (right) ....................................... 16

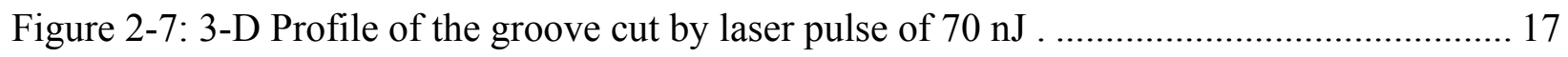

Figure 2-8:SEM Picture after treatment $\mathrm{Fe}_{0.6} \mathrm{Co}_{0.4} / \mathrm{SiO}_{2}$ multilayer fabricated with one laser pulse: without beam homogenizer (left); with beam homogenizer (right) ....................... 17

Figure 2-9: Non-convex and non-spherical lens shapes (top); and arrays of microlenses (bottom)

Figure 3-1: Materials used in the test article fabrication: Copper ring (top-left), silicone adhesive (top-right), optical flat (bottom-left), thin film (bottom-right)....................................... 21

Figure 3-2: Cured sample prior to removal of adhesive tape.............................................. 22

Figure 3-3: a) Two-sided polyester (left); polyimide (center); single-sided polyester (right) and b) with humidity control showing the reflected camera image ........................................... 22

Figure 3-4: Schematic of the excimer laser system. …................................................... 25

Figure 3-5: Excimer laser and associated instrumentation ............................................. 26

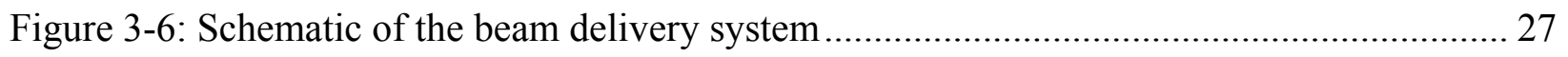

Figure 3-7: Laser pulse shape variation as a function of lens position ................................. 30 
Figure 3-8: Laser pulse shape variation as a function of beam splitter transmission value for constant pulse energy of $\mathrm{mJ}(\mathrm{Left}-60 \%$, Middle $-42 \%$, Right $-30 \%)$......................... 30

Figure 3-9: Microscopic view of the aperture through which the laser beam passed; used to

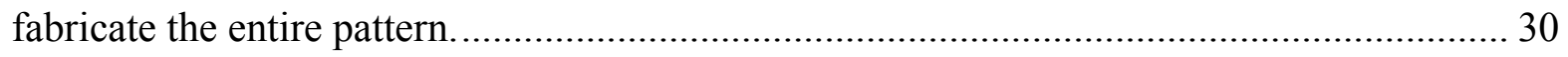

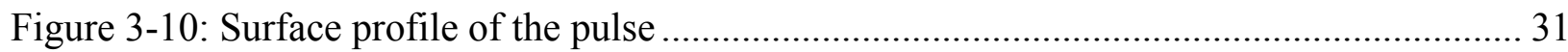

Figure 3-11: Surface profile of the pulse at another position on the pattern ........................... 31

Figure 3-12: The laser pulses shown overlapping to form a cross (The rectangular shape of the pulse immediately to the left of the vertical stripe is due to overlapping of the pulses)....... 33

Figure 3-13: Magnified view of selected features fabricated on the thin-film mirror ................ 33

Figure 3-14: Sample test pattern comprised of geometric features listed in Table 3-2 ............. 34

Figure 4-1: Geometry, node locations and coordinate system for SHELL91 elements. ............. 36

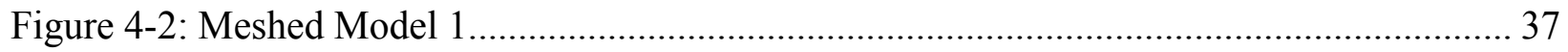

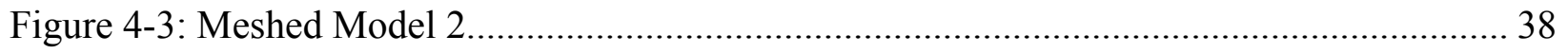

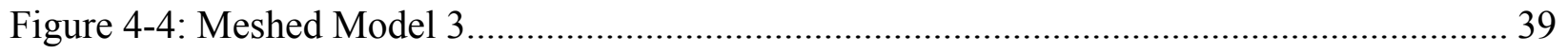

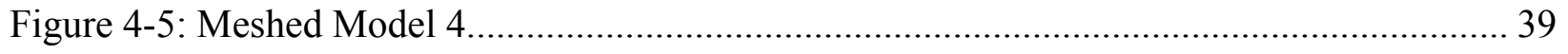

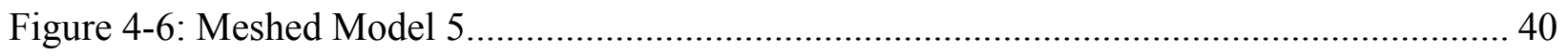

Figure 4-7: Zoomed in Model 5 (Area of non-metallization) ............................................... 41

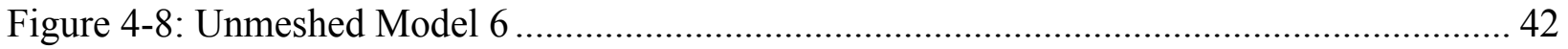

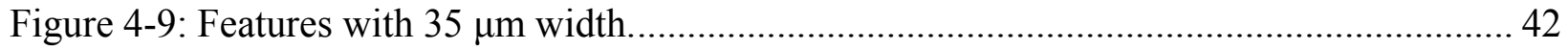

Figure 4-10: Variation in the Fundamental Frequency as a Function of the Metallized Area. .... 45

Figure 4-11: In-plane displacement (m) in the $\mathrm{x}$-direction (Top) and $\mathrm{y}$-direction (Bottom) for the application of $1.962 \mathrm{~N}$ force at the center.

Figure 4-12: In-plane displacement (m) in x-direction (Top) and y-direction (Bottom) for the application of $9.81 \mathrm{~N}$ force at the center of the mirror.............................................. 48

Figure 4-13: Out-of-plane displacement $(\mathrm{m})$ of the thin-film mirror with the application of 9.81

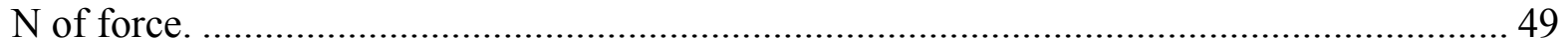

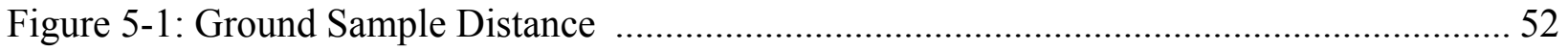


Figure 5-2: The projection and distortion of the real world 3D scene on to the image sensor .... 54

Figure 5-3: Four of the ten images of the calibration slide used for field calibration 55

Figure 5-4: 3D location of a point using Triangulation using a linear CCD array with two

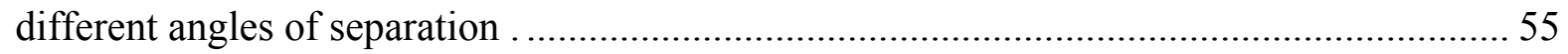

Figure 5-5: Illustration of auto- and manual-selected targets. .............................................. 57

Figure 5-6: The sub-pixel target marker. Note that the center of the calculated peak does not necessarily coincide with the center of a pixel. ............................................................ 58

Figure 5-7: Photographs taken for photogrammetry analysis at force level of 8 N.................. 60

Figure 5-8: Photograph marked with lines that were used to quantify the in-plane displacement of thin-film mirrors.

Figure 5-9: 3D reconstruction of the thin-film mirror using photogrammetry when no force was

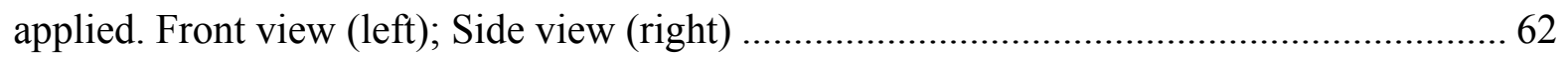

Figure 5-10: 3D reconstruction of the thin-film mirror using photogrammetry when force of $10 \mathrm{~N}$

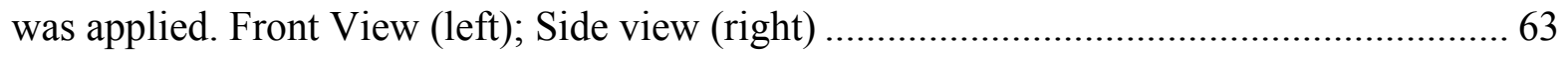

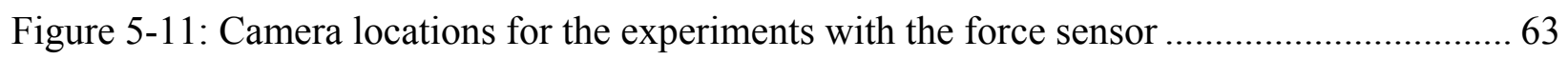

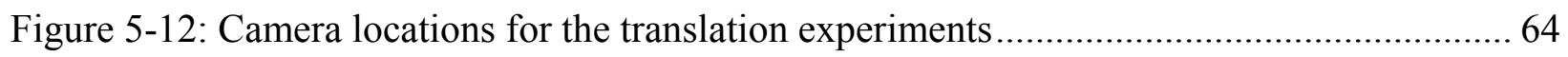

Figure 6-1: Sony XC-55 BB camera used to take photographs of the statically displaced thin-film mirror 66

Figure 6-2: Experimental set-up for the in-plane displacement measurement of thin-film mirrors showing the camera and the test article (top), and detail of the load cell (mounted on a linear stage) forcing the test article (bottom).

Figure 6-3: SONY XC - 55BB camera, standard 12 inch ruler, 0.75" X 1.00" paper (left), and calibration slide (right) imaged during the calibration process. 68

Figure 6-4: Thin-film mirror with the crossed wires used as reference points...... 69

Figure 6-5: One of the photographs from the final data set. Initial images were similar, but without the reference wires. 70

Figure 6-6: Photographs from one camera location for force different force levels: a) $0 \mathrm{~N}$; b) 2 N; c) $6 \mathrm{~N}$; d) $8 \mathrm{~N}$. 72 
Figure 6-7: Comparison between experimental and ANSYS results for line 2 ...................... 73

Figure 6-8: Comparison between experimental and ANSYS results for line 5 ....................... 73

Figure 6-9: Comparison between experimental and ANSYS results for line 8 ....................... 74

Figure 6-10: Comparison between experimental and ANSYS results for line $14 \ldots \ldots \ldots \ldots \ldots \ldots \ldots . . . . . . . . .74$

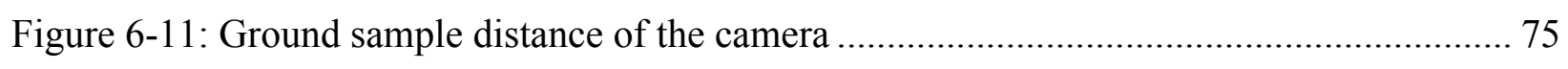

Figure 6-12: Experimental setup for the first translation experiment.................................... 77

Figure 6-13: Photograph of the thin-film mirror taken in the first translation experiment. Both dots and fabricated pattern were used as targets. ...................................................... 77

Figure 6-14: Photograph of the thin-film mirror taken in the third translation experiment ........ 79

Figure 6-15: Results of the first translation experiment ................................................... 80

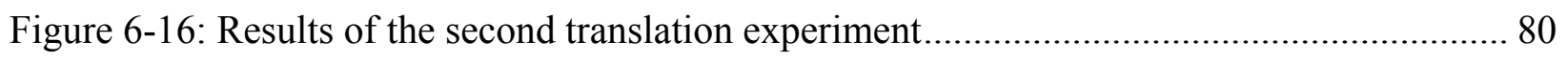

Figure 6-17: Results of the third translation experiment ............................................... 81

Figure 6-18: Frames showing deformation patterns resembling second mode ........................ 82 


\section{LIST OF FILES}

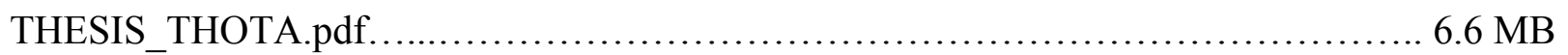




\section{Chapter 1: INTRODUCTION}

\subsection{Introduction}

Since the early days of mankind, the possibility of exploration of the celestial bodies has fascinated people of all cultures. They always dreamed of having routine access to space to explore the mysterious, wonderful and never ending universe. While access to space has become almost routine, the high cost of launch is a major obstacle preventing more frequent visits to space. Spacecraft that are smaller, cheaper and lighter are currently being pursued as a partial solution to this problem.

Payload mass and volume play a significant role in designing a space vehicle. Maximizing spacecraft performance while significantly reducing its weight remains a challenge to space organizations. One proposed solution to this problem is the advent of ultra-lightweight spacecraft called "Gossamer Space Structures." These structures have a high strength to mass ratio which reduce the payload mass and volume and make the launch economical. Gossamer space structures include solar sails [Figure 1-1], antenna arrays [Figure 1-2], solar shields, optical mirrors, entry and landing systems, habitats etc. Development of several crucial technologies [Figure 1-3] will allow the full-fledged deployment of ultra-lightweight structures in space. Among the six technologies shown in Figure 1-3, development and verification of modeling and control technologies are considered the most crucial, and are addressed in this thesis.

Performance of ultra-lightweight spacecraft depends on their design and control, which further depend on their motion upon excitation. Some of these ultra-lightweight structures are coated with aluminum or gold for special applications such as solar sails, sunshades, optical mirrors etc. Precision control of the surface shape is vital requirement for the success of such applications. Gossamer space structures are extremely compliant and flexible, which complicates ground testing in full scale due to the effects of gravity loading, and in ambient atmospheric conditions due to aerodynamic loading. One approach to counter these problems is to design and construct a set of smaller test articles for testing. Since the early days of NASA, ground testing and replica scale model testing has been performed with great success, allowing results to be scaled up to predict the performance of the actual structure $[1,2,3]$. 
One method of evaluating the dynamic performance a Gossamer structure is to measure displacement at various locations on its surface as it moves. Both in-plane and out-of-plane displacements are required to characterize the dynamic response of the surface of these structures. Although many well-developed methods for measurement of out-of-plane surface motion exist, fewer options for tracking the in-plane component of surface motion are currently available. Hence, the development of experimental techniques that enable the measurement of inplane surface motion, vital for validation of computational models and for control, are necessary for the successful deployment of ultra-light structures in space.

The test articles in this work are not scale models of any particular full-scale structures, but were designed to ascertain experimental limitations and to achieve the objectives discussed later in this chapter. Though scale modeling is not a part of the work, the performance of the experimental models developed can be used to verify computer simulations of the response of scale and full-size models. Once computational tools and control strategies have been verified experimentally by monitoring the surface motions, similar computational and experimental studies on scale models can be used to predict and control the dynamic performance of full-scale space structures.

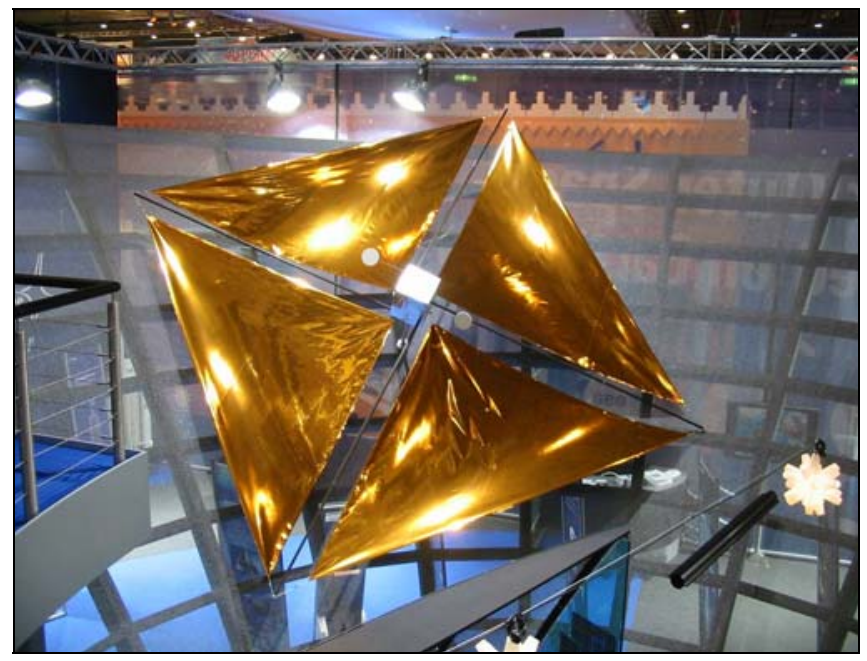

Figure 1-1: Model of a solar sail [4]. 


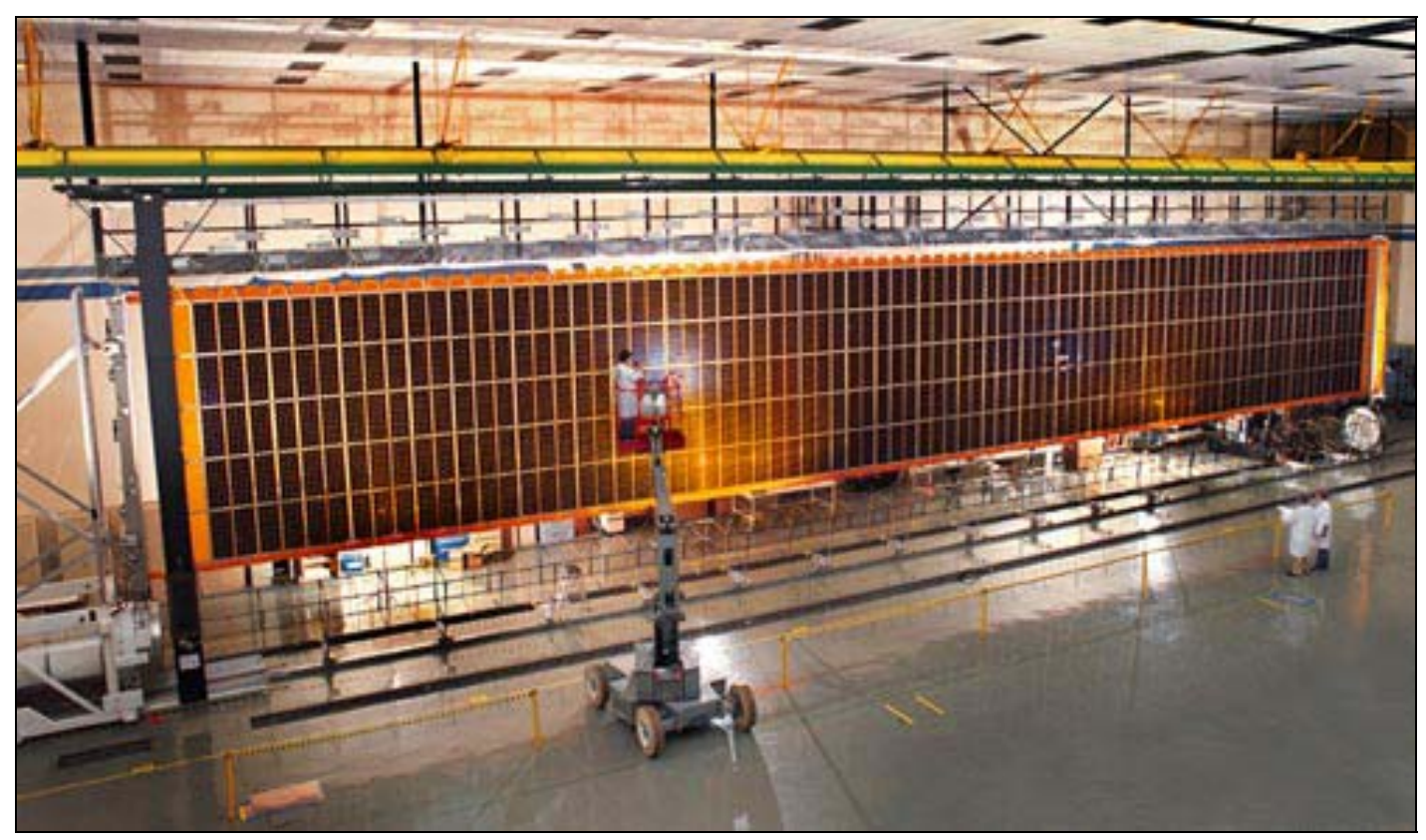

Figure 1-2: Model of a solar array in Lockheed Martin [5].

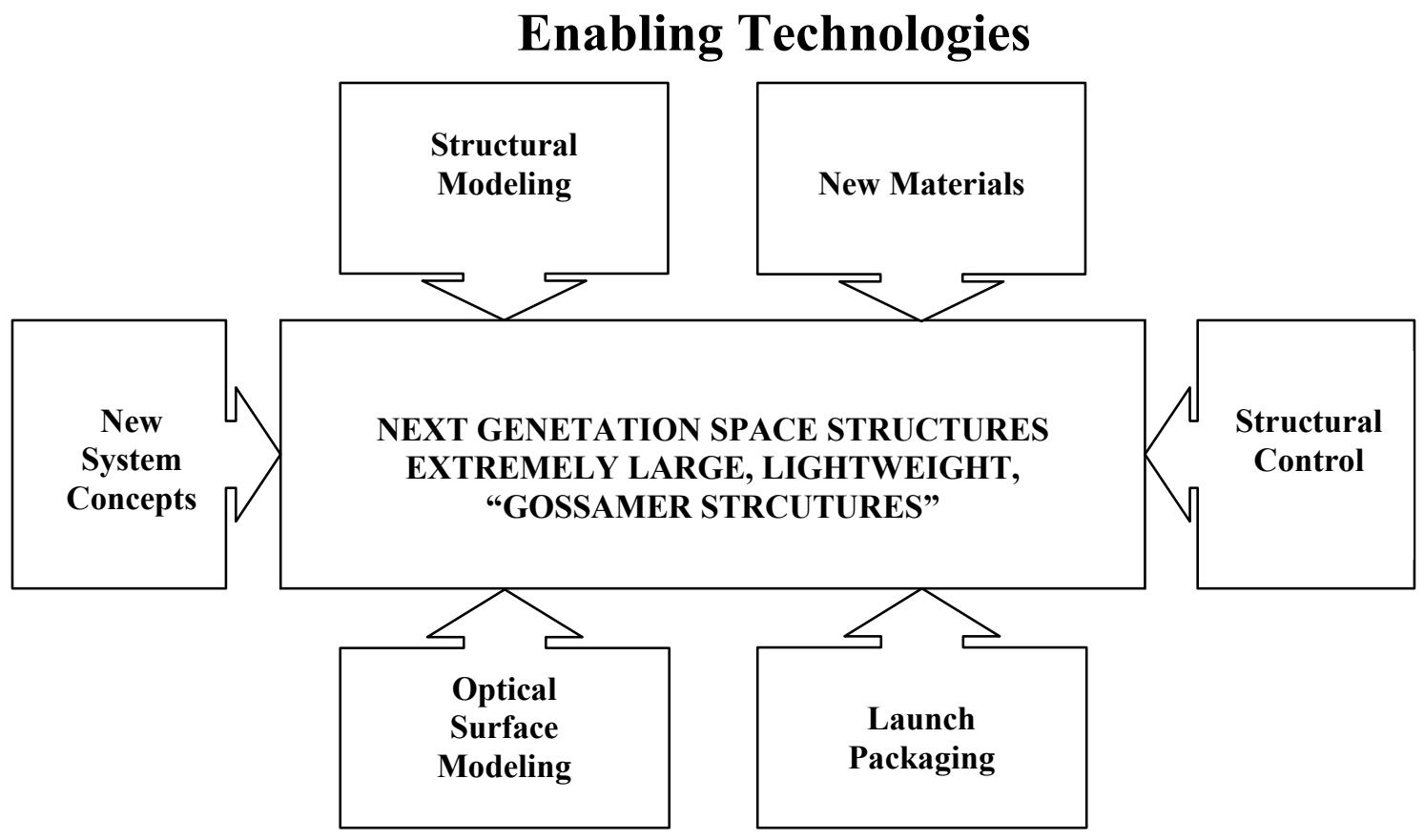

Figure 1-3: Crucial technologies for the development of ultra-lightweight structures 
Measurement of surface displacement of thin-film structures must be performed using non-contacting techniques. This is because the installation of contacting sensors would dramatically change the dynamic characteristics of the surface under observation. Most noncontacting in-plane displacement measurements require a non-reflective surface, or one that has been specially patterned. This means that to perform the desired in-plane measurements, the surface of the thin-film structure itself will need to be modified to allow the verification of measurements. The surface configuration chosen will therefore become an integral part of the measurement and must be given careful consideration.

\subsection{Objectives of Thesis}

The main objective of this thesis is to develop a system to perform surface measurements of in-plane motions of small-scale thin-film structures, similar to those proposed as "Gossamer Space Structures" such as optical reflectors, solar sails, sunshades, etc. The following specific objectives were defined for this thesis:

1. To define an optimal surface pattern suitable for in-plane displacement tracking that has minimal influence on the dynamic response of the thin film

2. To evaluate fabrication processes for surface patterns, and

3. To establish the basis for a precision and robust measurement technology for in-plane motion of thin-film structures

Although this thesis is primarily experimental, computer simulations of computational models are used in conjunction with the experimental results throughout.

\subsection{Thesis Summary}

Chapter 2 presents a literature survey of material relevant to this work, including displacement measuring techniques, photogrammetry and the laser ablation technique used for pattern fabrication on the surface of the thin-film mirrors used as test articles. Chapter 3 describes the fabrication of the test articles used for the thesis and effect of environmental conditions such as temperature and humidity. A Krypton Fluoride (KrF) excimer laser was used to fabricate the pattern on the test article via the process of laser ablation. Laser parameters were set to produce a pattern for maximum in-plane resolution and minimum impact on the dynamic response on the thin film structure. The effect of the pattern features on the dynamic response of 
the thin film mirrors was studied using the finite elements models discussed in Chapter 4. Establishing that the patterns did not affect the film dynamics is vital, as the use of traditional contacting sensors for the surface measurement was ruled out due to their significant affect on dynamic response. Normal-mode analysis of the thin film mirrors was done using various finite element models in order to study the effect of size, geometry and pattern configuration. Chapter 5 introduces with a technique called photogrammetry, which is used to perform full-field threedimensional measurement of surface displacement using multiple photographs. Chapter 6 deals with the displacement measurement of a test article under static deformation that was done as a part of determining feasibility of the system for future dynamic tests. Three-dimensional models of the displaced fabricated pattern were also created using the process of photogrammetry. Chapter 7 deals with the summary, conclusions of this thesis as well as future work. Conclusions were drawn individually from each part of the work and accumulated in this chapter.

The significance of this thesis is to establish basis for an important sensing technology that addresses the needs of scientists and engineers performing research and development of ultra-light structures for future space missions. The development of this technology will complement numerous other projects related to the modeling, testing and control of thin-film structures.

Copyright $(\mathbb{C}$ Phanikrishna Thota 2003 


\section{Chapter 2： LITERATURE REVIEW}

\subsection{Partial History of Ultra-light-weight Space Structures}

Ultra-light-weight space structures have been of interest for about 50 years because of their potential low cost, exceptional packaging efficiency, and deployment reliability. Their packing configuration can be varied to accommodate the geometry of the available launch container [6].

Goodyear Corporation was the first to develop inflatable deployable space structures. It developed search radar antennae, radar calibration spheres and lenticular inflatable parabolic reflectors during 1950's and mid-1960's [7]. In the late 1950's and early 1960's, flights of echo balloons demonstrated capabilities for the development, fabrication and launch of large, highprecision inflatable space structures. In the late 1960's and early 1970's, L'Garde, Inc., successfully flew a large number of various lightweight re-entry vehicle decoys and singlestructure vehicles in suborbital flights. In the early 1980's, the European Space Agency (ESA) built a 6-m reflector antenna, which was a 1/3-scale model of a VLBI (Very Large Baseline Interferometer). ILC Dover has pioneered the development and manufacturing of structures such as large aerostats, inflatable ground structures, inflatable rigidizable space structures, Mars Pathfinder landing airbags, and the Next Generation Space Telescope (NGST) deployable sunshade. ILC Dover also developed technology for building a 3x10 m inflatable deployable rigidizable solar-array system. Although progress has been made, challenges still exist in areas related to:

- Handling, processing and manufacturing high precision thin-film assemblies

- Metallization of thin films

- Determination of the characteristics of high precision reflector structures via analytical, numerical and experimental methods.

- Performing surface measurements of membrane dynamic response

NASA's interest in demonstrating the potential of this relatively new class of space structures resulted in their sponsoring the IN-STEP inflatable antenna experiment, which flew on STS-77 on May 29, 1996 [8,9,10,11]. 


\subsection{Techniques for Measuring In-plane Displacements}

Different measurement schemes, including Moiré Interferometry, Electronic Laser Speckle Interferometry and Optical Feature Tracking with Digital Image Processing all require different surface geometries, and all possess different measurement ranges and sensitivities that in fact depend on the size of the surface features fabricated. Moiré interferometry and electronic laser speckle interferometry are most often applied for determination of surface displacements in static, rather than dynamic loading situations. The challenge undertaken here is a dynamic one, so the evaluation of these methods will be focused on dynamic applications.

\subsubsection{Moiré Interferometry}

This technique was developed in 1980's and is capable of providing whole-field maps of in-plane surface displacements. Figure 2-1 shows the schematic representation of elements of such a system. The constructive and destructive interference of either of the beams and their interaction with a diffraction grating of horizontal lines embossed on the specimen produce moiré fringe patterns, which depict the surface displacement field. The displacement sensitivity of this method depends on the line frequency spacing on the grating. This method requires a surface treatment of the object under evaluation either by chemical etching or by molding an epoxy layer containing the grating. Either of these could change the dynamic response of the thin-film structures under study. This method can be applied to study the dynamic response of composites, metal plasticity and fracture mechanics (Figure 2-2). High-density phase diffraction gratings are typically used to reveal in-plane displacement components with a sensitivity of 0.4 micron/fringe [12]. 


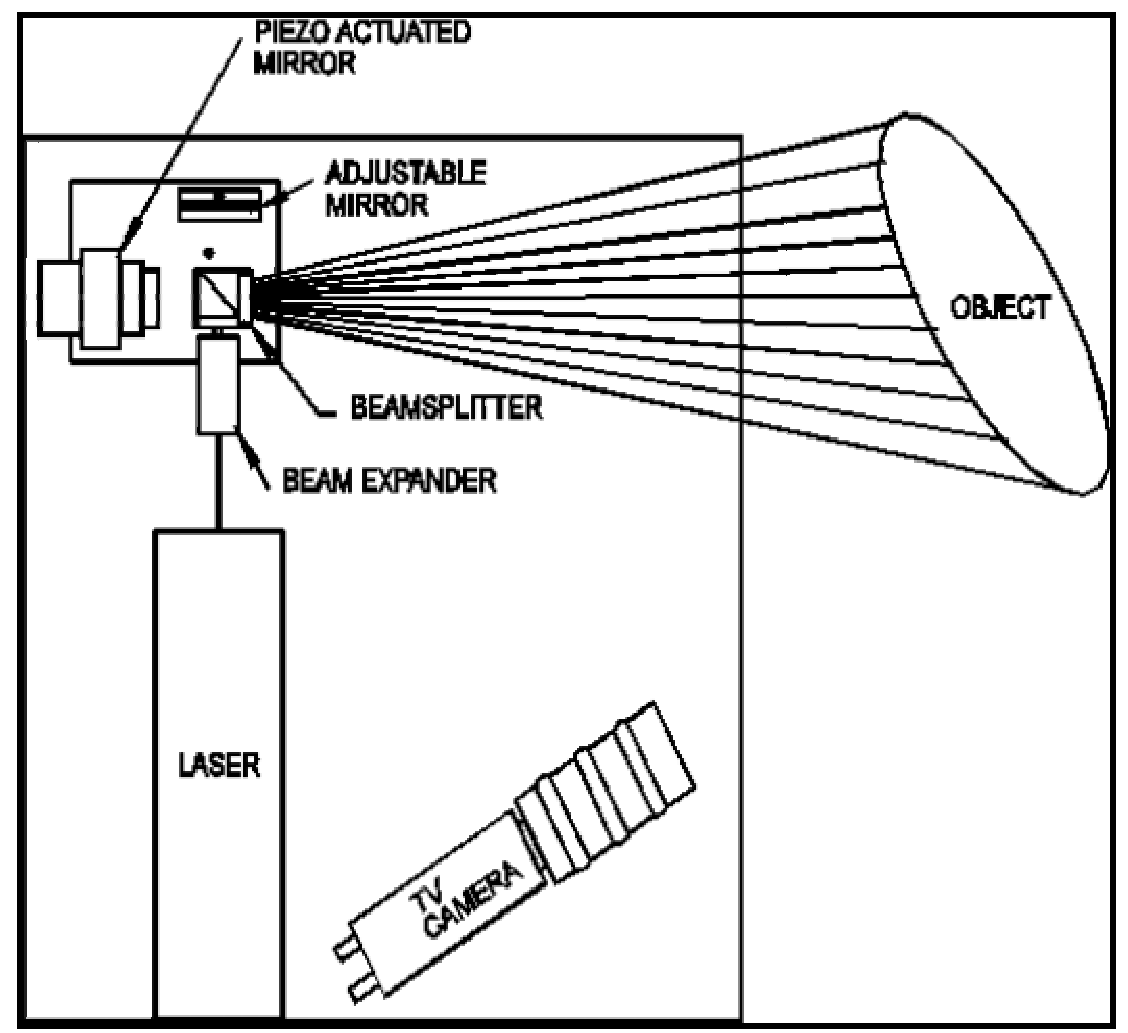

Figure 2-1: Schematic representation of the optical system required for Moiré interferometry [13].

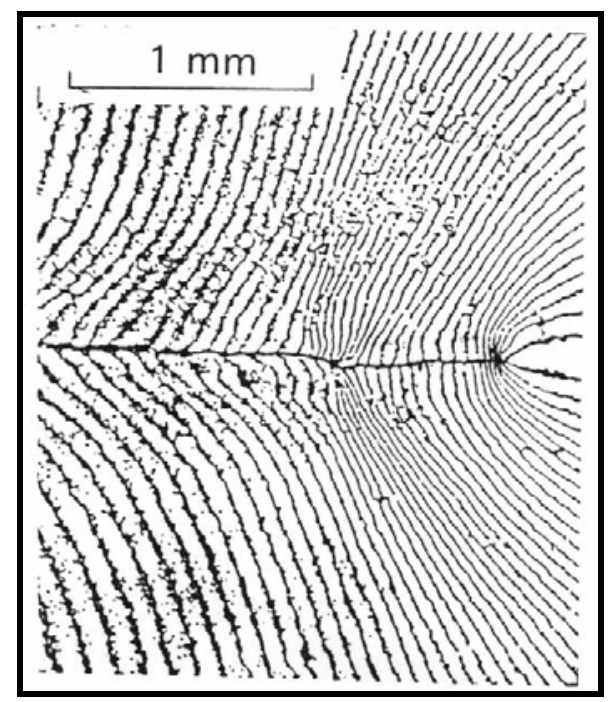

Figure 2-2: Moiré Interferometry fringe pattern about a fatigue crack [14]. 


\subsubsection{Laser Speckle Interferometry}

Also known as electronic speckle pattern interferometry (ESPI), this is another laserbased technique that allows the performance of full-field, non-contact measurements of both inplane and out-of-plane surface displacements. A significant advantage of speckle interferometry over Moiré interferometry is that no special surface preparation or configuration is required, as long as the surface under observation is optically rough (non reflective). When a laser beam is directed towards such a surface, speckles occur due to the interference caused by the interaction of the coherent beam with the surface features (Figure 2-3, Figure 2-4). This method exploits the fact that speckle intensity varies randomly on the rough surface target. This random variation is due to the high laser coherence light used to illuminate rough surface target. The principal kinds of measurements which are made using ESPI are in-plane and out-of-plane displacements. The first of these requires two plane wavefronts to be incident at equal and opposite angles to the object surface. The second case requires an in-line smooth reference beam [15].

Hansen used a compact ESPI system for displacement of specular reflecting or optically rough surfaces [16]. He measured the displacement of a steel membrane of a capacitor microphone when a bias voltage is applied to it. A glass plate was used to scatter the incident light towards the object surface and a reference glass plate was used to establish a reference wave. The reference wave was established by reflecting a small fraction of the diffuse illumination from the glass plate just in front of the object towards the camera. Figure 2-3 and Figure 2-4 show the speckles on the undeformed and deformed steel membrane respectively. The steel membrane as well as the reference glass plate in front of the microphone reflects the diffuse light scattered from the ground glass plate. Speckles are therefore seen both on the membrane and outside of the microphone. 


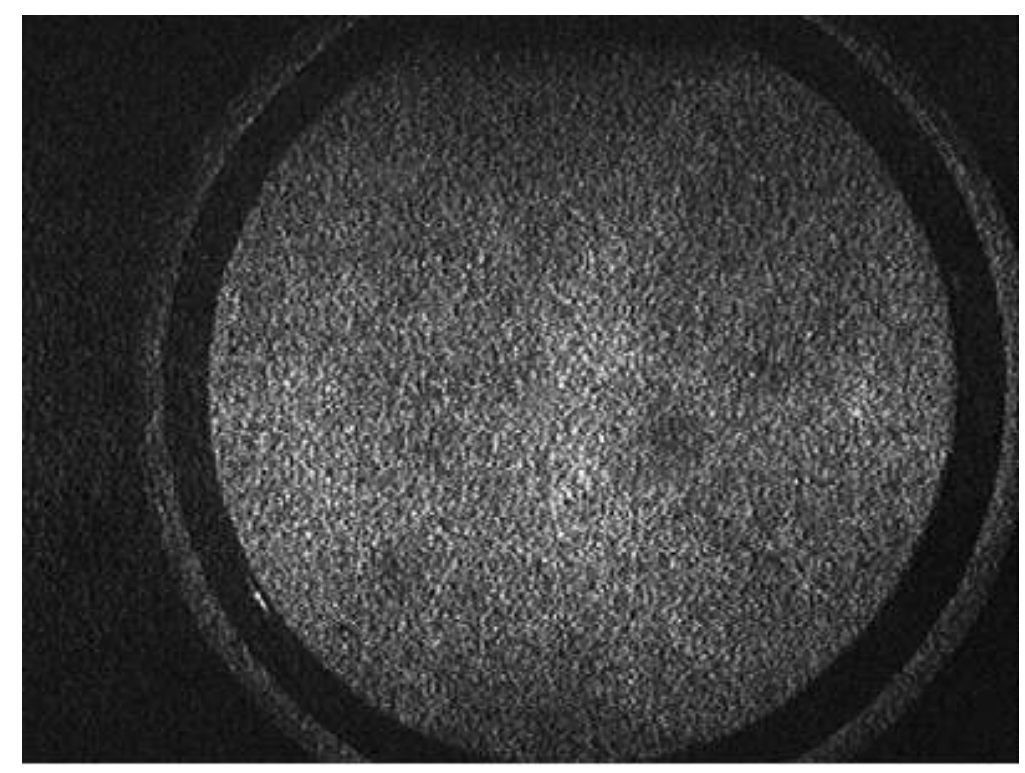

Figure 2-3: Speckles on both the undeformed membrane and outside of the microphone [16].

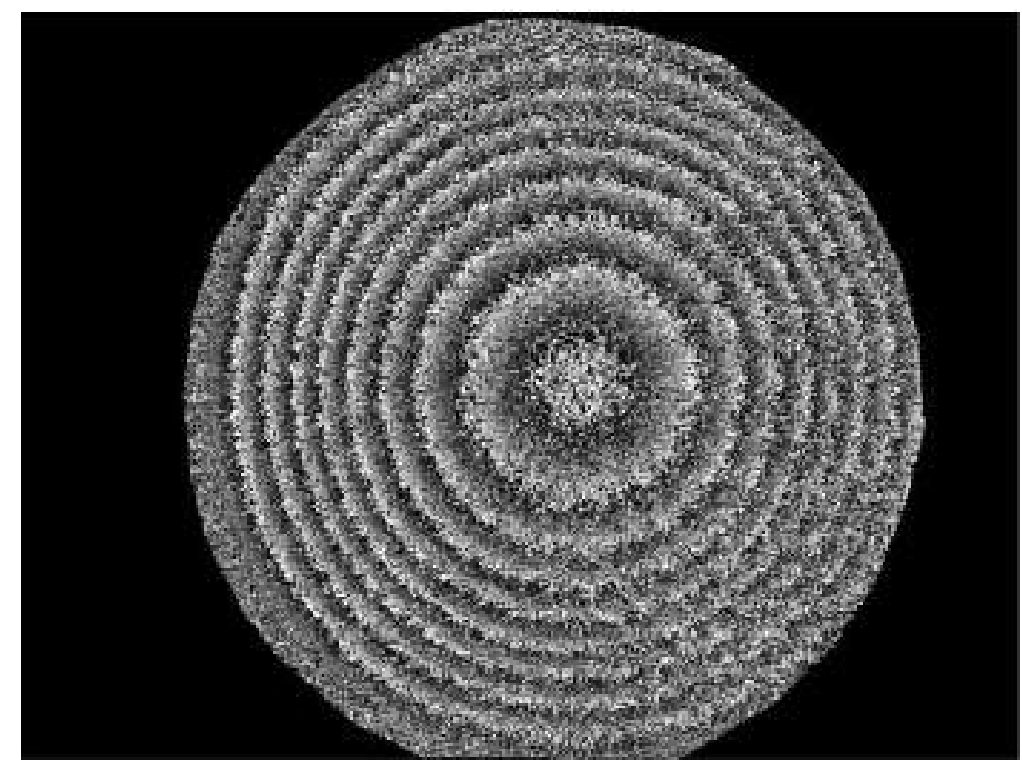

Figure 2-4: The interferogram of the steel membrane as it bends from its equilibrium position due to application of an electrostatic force [16].

As the surface displaces, the pattern of speckles changes. A comparison of the displaced and reference speckle pattern images allows the displacement field to be reconstructed. Many researches have used this method to measure the surface of vibrating PZT piezoelectric disks, polymethyl methacrylate, etc. $[17,18,19]$. 
Although speckle interferometry does not require a specific surface preparation, it is not especially well suited for the applications described herein. This is because the desired surface of the thin-film reflectors under consideration for use in many Gossamer structures is optically smooth, and will require roughening in order to permit interrogation by this method. As with Moiré interferometry, the surface could be prepared by either a laser or chemical etching process, or could be coated to provide the required rough surface.

\subsubsection{Optical Feature Tracking}

Moiré and speckle interferometry both provide measurements of in-plane displacements of a fraction of a micron to a few microns. But for dynamic measurements, optical tracking is better suited. Mazza et al. used optical tracking for deformation measurements in microbars with nanometer resolution [20]. Toigo et al. performed high-resolution cloud feature tracking on Venus by the spacecraft Galileo, attaining a spatial resolution of $15 \mathrm{~km}$ [21]. In this method a sequence of images of the patterned surface was recorded. A feature in the image, such as an intersection of an etched grating pattern, was located in each frame of the sequence and tracked to create a two-dimensional displacement time history. Researchers at NASA Glenn Research Center applied this approach to the analysis of images generated by microgravity combustion and fluid physics experiments [22]. The key to applying this approach for in-plane measurements is therefore to acquire a sequence of images of a moving surface with sufficient resolution to distinguish a target feature for tracking [23].

\subsubsection{Feature Selection}

Feature selection is an important aspect of any tracking algorithm design. In addition, no feature-based vision system can work unless good features can be identified and tracked from frame to frame. Shi and Tomasi proposed a feature selection criterion that is optimal by its construction because it is based on how the tracker works [24]. They also tested the efficiency of the feature selection process and the tracker system with several simulations and experiments. They monitored the quality of a feature by a parameter called dissimilarity, which quantifies the change of appearance of a feature between the first and the current frame. Dissimilarity is the feature's rms residue between the two frames of interest. The feature is abandoned if the dissimilarity between two frames is above a predefined threshold value [24]. 
The Kanade-Lucas-Tomasi (KLT) feature tracking algorithm selects the best features in an image and then tracks those features over a time sequence of frames. Assuming the image is digital, the KLT algorithm selects the best features to track in the following way. Each pixel is assigned a value and the features are selected by taking " $n$ " pixels with the highest value, where " $n$ " is the number of tracking features desired. To calculate the value of each pixel, a $2 \times 2$ gradient matrix of a small window surrounding the pixel is calculated, and the corresponding eigenvalues are computed. For a feature to be good for tracking, there must be a high degree of intensity variation across the window. If the eigenvalues are both low, then the window has little variation. One high and one low eigenvalue represent a smooth change in gradient, or edge. Two high eigenvalues means there is large variation, such as a corner or rough area easily picked out of the textured pattern. In this case, the pixel is selected if the minimum of the eigenvalues is above a predefined threshold [24].

\subsubsection{Other Optical Methods}

Rembe et al. in their paper [25] investigated optical measurement methods for studying dynamic behavior in MEMS. They used a Stroboscopic Microscopic Interferometer System (SMIS) developed at BSAC (The Berkeley Sensor \& Actuator Center). The system can measure both in-plane and out-of-plane motions in a single experiment. In-plane motions were measured with sub-pixel resolutions to better than $5 \mathrm{~nm}$. They measured the shape of the moving specimen, frozen by the strobe light, using phase-shifting interferometry (PSI). Stroboscopy and digital-image processing were employed to determine in-plane motions with sub-pixel resolution. Another system was developed at MIT and was called a "Computer-Micro-VisionSystem" (CMVS). It employed stroboscopy and image-processing techniques to track the motions of moving structures. Through a focus variation, this system can measure out-of-plane as well as in-plane motions [25]. Both SMIS and CMVS can only be used to characterize MEMS motions when the dynamic processes are periodic or periodically repeatable. In addition, it must be possible to synchronize the strobe light with the device motion. Therefore, SMIS and CMVS are not applicable to the measurement of noisy or transient motions. 


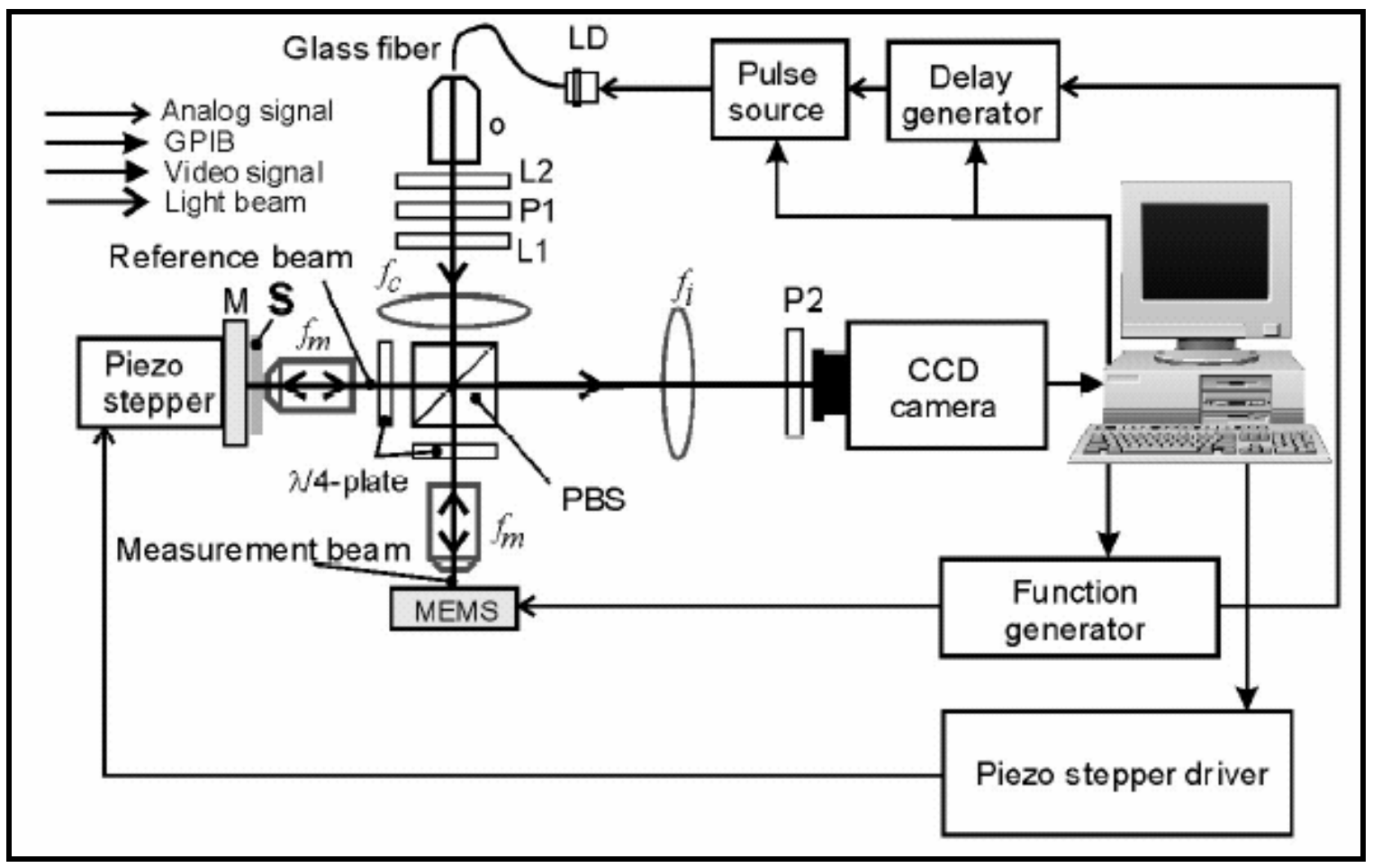

Figure 2-5: Schematic of Stroboscopic Interferometer System [25].

Of the wide range of optical techniques available, optical tracking using photogrammetry was selected as a robust and efficient method for evaluating the dynamics of thin-film structures. This is because photogrammetry uses a $3 \mathrm{D}$ image correlation that is substantially more robust and has greater dynamic range than other full-field imaging technologies such as ESPI and Moiré interferometry. Photogrammetry tracks changes in an applied pattern with subpixel accuracy, and thus does not suffer from the speckle decorrelation limitations of ESPI [26].

\subsection{Review of Thin-Film Dynamics and Measurement}

\subsubsection{Dynamics of Inflatable Structures}

The idea of using thin films for space applications within inflatable structures was first developed in 1962 by Frei Otto [27], who published ideas for inflated tubular frames for use in structures such as orbiting platforms. Even though several papers on static structural analysis of inflated cylinders have been written [28,29,30,31,32,33,34,35,36], very little work has been done until recently to study the dynamics of these kinds of structures. Main et al. contributed significantly to this field with their paper describing results of modal tests of inflatable cantilever beams and the determination of effective material properties [37]. Slade et al. investigated the 
dynamics of polyimide thin-film inflated cylinders and described dynamic tests and potential applications of inflatable solar concentrators [38].

\subsubsection{Static Measurement of Thin-film Surfaces}

Dharamshi et al. compared static displacement measurements of an aluminized, $0.5 \mathrm{~m}$ square Kapton ${ }^{\circledR}$ membrane obtained using photogrammetry and the use of a conventional scanning capacitance type displacement sensor [39]. Jenkins et al. used the capacitor sensor to study wrinkling in a membrane that was subjected to tensile and shear loads [40,41] and also studied the surface profile of an inflated membrane. Blandino et al. used a capacitance sensor to study the wrinkling near the corners of a $0.5 \mathrm{~m}$ square Kapton ${ }^{\circledR}$ membrane [42]. The major drawback of capacitive sensors is that they measure displacement over a small area, and must be scanned over an entire surface in order to obtain a full-field measurement. This renders this approach ill-suited for dynamic measurement. In addition, capacitive measurements are based on the perpendicular distance between two surfaces, and hence permit measurement of only the outof-plane displacement component.

Black et al. studied the out-of-plane displacements and performed dynamic shape measurement using a time series of images. They expanded the method of photogrammetry to multiple time steps, enabling the object under study to be characterized dynamically. This process has been termed "Videogrammetry." Black et al. characterized the dynamic behavior, such as deployment and vibration by tracking the overall shape of the object under consideration. They used the dot-projection method, in which dots are projected on to the surface of the thinfilm structure and their 3D coordinates are calculated using time sequences of images [43]. This method cannot be used to quantify the dynamic in-plane displacement. Pappa et al. compared the dot projection method with the use of retroreflective targets for dynamic shape measurement of thin-film membranes through videogrammetry. While dot projection does not dynamically load the membrane the way adhesive-backed retroreflective targets do, projected targets are difficult to use with highly reflective and transparent membranes. They concluded their paper by suggesting a laser-induced fluorescent dye could be incorporated into the membrane during manufacturing, and used for measuring the in-plane as well as out-of-plane displacements [44]. In general, target projection is ill-suited for in-plane displacement measurements since the dots do not translate with the membrane as it moves within its plane. The displacement technique 
developed in this research involves the use of an integrated surface pattern; hence it allows both out-of-plane and in-plane dynamic measurements [44].

\subsection{Laser Ablation for Surface Patterning}

Procedures used to prepare a film surface for patterning depend on the surface features chosen. Possibilities include creating a pattern in the metallization surface via a laser or chemical etching technique, printing a pattern on the surface, molding an epoxy layer containing the pattern to the surface, or incorporating the pattern into the polymer film as the film itself is fabricated. Laser etching seemed to be a convenient as well as an economical way of producing the patterns evaluated herein, as the facilities for performing this operation are readily available at UK.

Ablation is the process of removing a small area of metallization on a target surface using a laser of high energy density. Ablation is more than just evaporating the metal coating, it implies that energy is penetrating the layers beyond the surface, and that the material leaving the surface is moving away with a great deal of thermal and kinetic energy. Evaporation is a more passive process that occurs closer to equilibrium. Ablation is a non-equilibrium process that happens very quickly (transient). The laser energy delivered to the sample converts a solid at room temperature into an expanding vapor plume within a fraction of a second.

The unique characteristics of the laser-matter interaction mechanisms of ultra-short laser pulse ablation makes sub-micron machining achievable with this process. This method of machining submicron features by ultra short laser pulses has been reported since 1995 $[45,46,47,48,49]$. Micromachining of thin metallic films has wide applications in high-density data storage and semi-conductor microelectronics. Venkatakrishnan et al. performed laser micromachining on 1000 nm-thick gold film using a femtosecond laser with a central wavelength of $400 \mathrm{~nm}$, pulse duration of $50 \mathrm{fs}$ and a $1 \mathrm{KHz}$ repetition rate. Various grooves were cut on a gold thin film with laser pulses of various energies, and their ablation depths were characterized as a function of pulse energy [50]. Pronko et al. machined submicron holes using a femtosecond laser of wavelength $800 \mathrm{~nm}$ [45]. Liu et al. and Kautek used a femtosecond pulse laser ablation process for metal films, as well as semi-conducting and ceramatic materials $[46,47,49]$. 
Laser ablation using an excimer laser on thin films has wide applications in lithography and rapid prototyping. Pfleging et al. studied laser micromachining using lasers for application in thin film technology. They patterned films with thicknesses varying from $100 \mathrm{~nm}$ to $2 \mu \mathrm{m}$ using excimer laser radiation with $248 \mathrm{~nm}$ wavelength. They studied the effects of film material, film thickness, laser energy (fluence) and number of pulses on the ablation process. They concluded that with an increase in film thickness the optimal laser energy (fluence) for patterning increases and also studied the patterning of metallic films as a function of various excimer laser operating conditions including the use of a beam homogenizer, mode of laser scan, etc [51]. Sometimes the laser energy applied may be so high that the ablation depth will be greater than the film thickness [51]. Rumbsy et al. studied the selective ablation of thin Al films on polymer substrates using one excimer laser shot [52].

Excellent UV absorption properties and highly non-thermal ablation behavior of polymers make them well-suited for surface structuring using an excimer laser, making the process a valuable micro-fabrication technology. Naessens et al. fabricated micro lenses in polymer layers with excimer laser ablation. A single circular shaped excimer laser beam was used in the fabrication of micro lenses [Figure 2-9]. Additional laser pulses were used to clear the debris from the lens to improve the surface finish to that of optical quality. A circular aperture was used to obtain a circular laser pulse. Besides fabricating a single micro lens, they also fabricated arrays of multiple micro lenses in an easy to set-up process, allowing great flexibility in terms of size, shape and focal length [53].
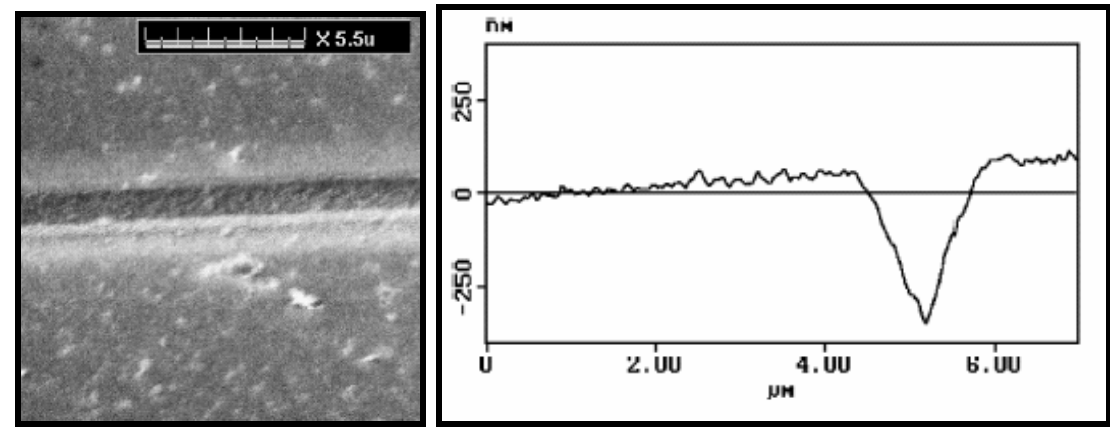

Figure 2-6: Groove ablated on gold thin film by femtosecond laser pulse of $70 \mathrm{~nJ}$ energy (left); Cross-section of the groove cut by laser pulse of 70nJ (right) [50]. 


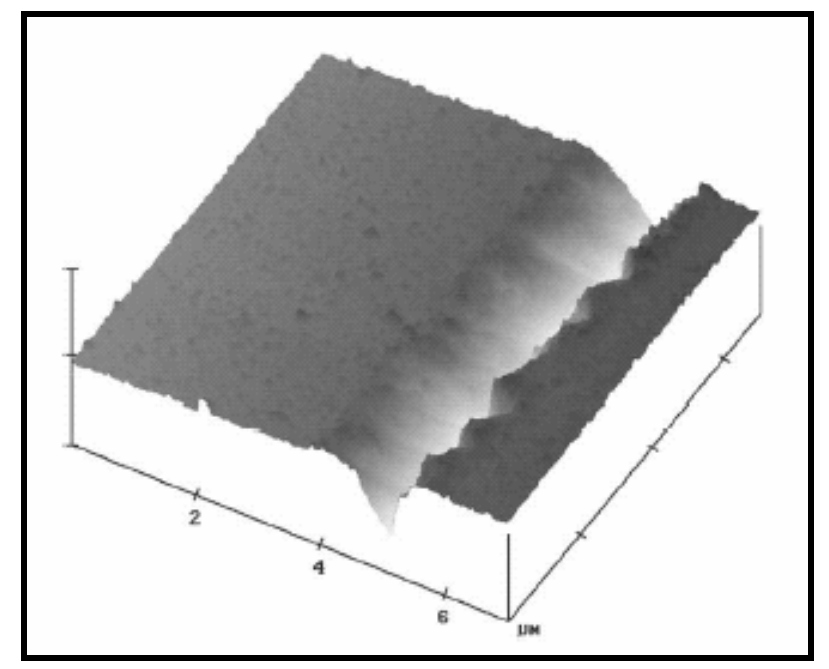

Figure 2-7: 3-D Profile of the groove cut by laser pulse of $70 \mathrm{~nJ}$ [50].

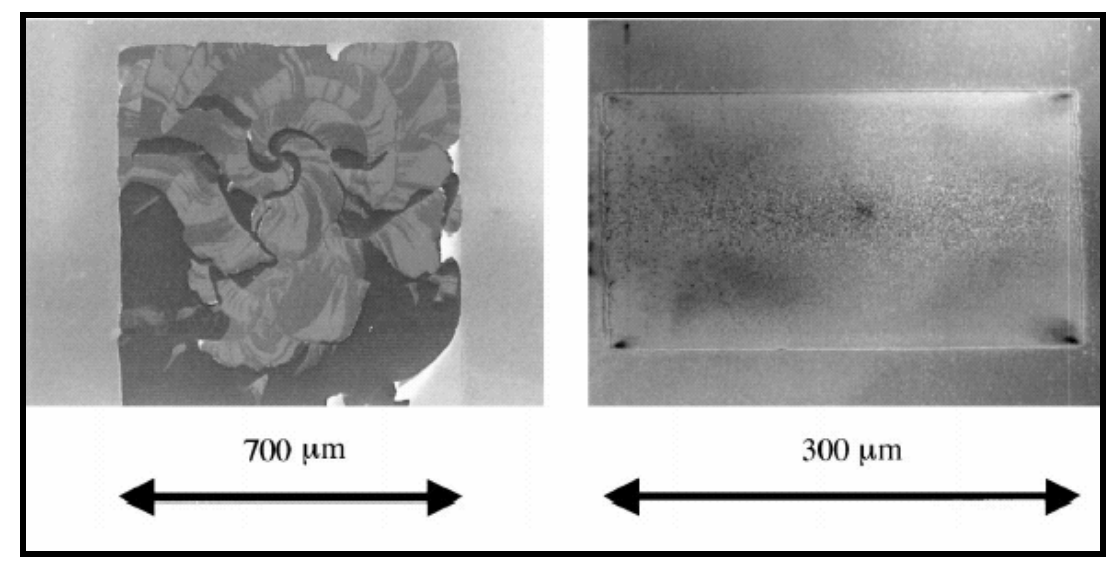

Figure 2-8:SEM Picture after treatment $\mathrm{Fe}_{0.6} \mathrm{Co}_{0.4} / \mathrm{SiO}_{2}$ multilayer fabricated with one laser pulse: without beam homogenizer (left); with beam homogenizer (right) [51]. 


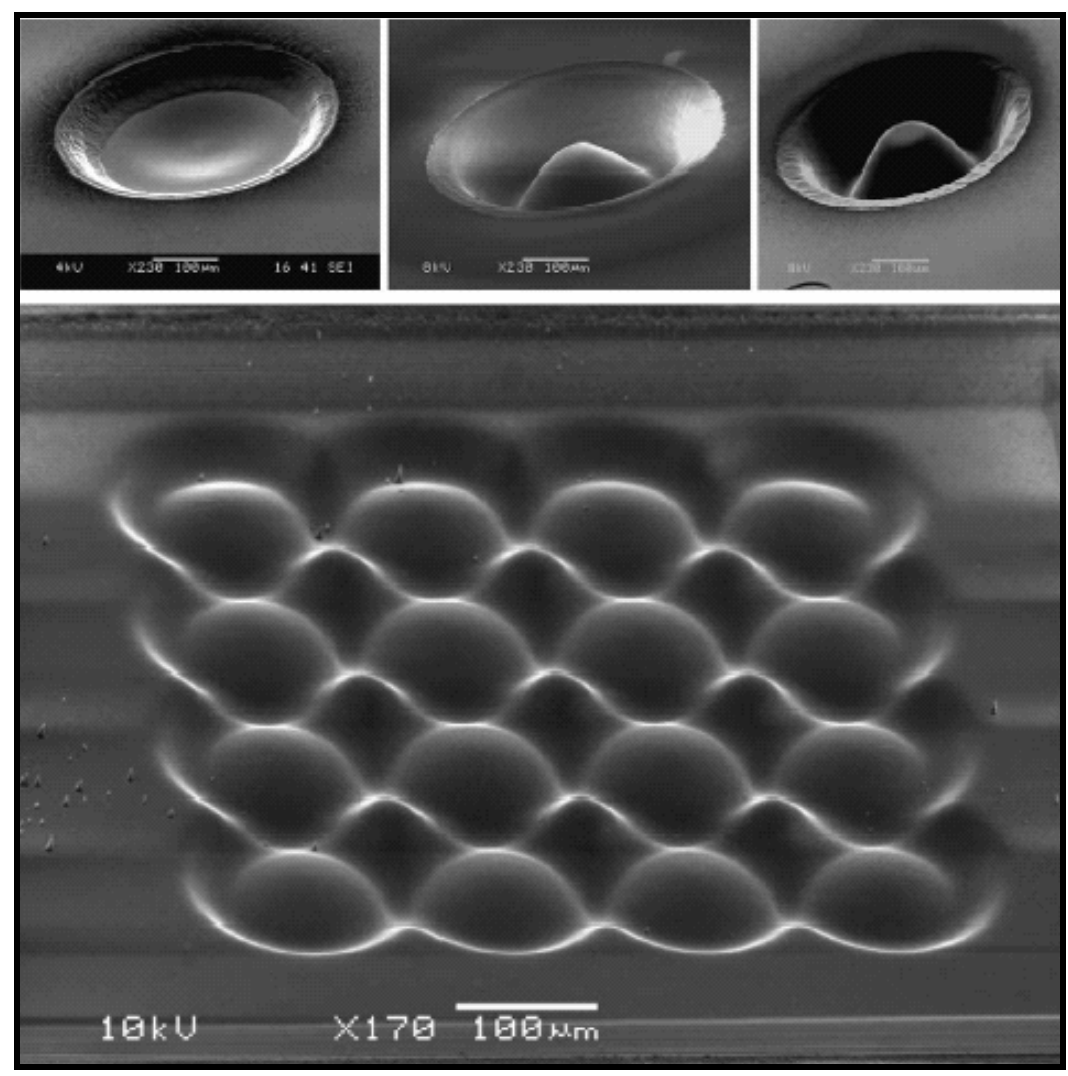

Figure 2-9: Non-convex and non-spherical lens shapes (top); and arrays of microlenses (bottom) [53]

Several technologies that include semiconductor circuitry [54], sensors [55], printing plates [56], tissue engineering [57] and micromechanical devices [58] have utilized similar surface patterning techniques. Callewaert et al. made micro patterns on polymeric surfaces using an excimer laser. The polymers were irradiated with a UV KrF excimer laser (wavelength=248 $\mathrm{nm})[59]$.

\subsection{Photogrammetry}

Photogrammetry is the science of measuring the size and location of three-dimensional objects using photographs [68]. The typical application of photogrammetry is creating aerial surveys and maps, a process that is known as topographic photogrammetry. It is also used in diverse ground-based applications such as archaeology, bioengineering, civil engineering, computer animation, forensic analysis, historical preservation, mechanical inspection, ship construction, etc [60]. Modern close-range photogrammetry uses digital imaging sensors and computer data analysis and often measures hundreds or thousands of object points in order to 
obtain a precise surface profile [61]. Photogrammetry offers the simplicity of taking photographs coupled with good-to-excellent measurement precision [62].

\subsubsection{History of Photogrammetry}

The early roots of photogrammetry can be traced back to the renaissance painters, particularly Leonardo da Vinci, who studied the principles involved in the geometric analysis of pictures in the late 1400's. The development of projective geometry, which forms the mathematical basis of photogrammetry from passive imaging systems, was followed by the production of usable photographs in the early 1800's by Niepce and Daguerre. The first suitable camera and procedure, metro photographic, for making photogrammetric measurements was developed in 1895 by Laussedat. For this accomplishment Laussedat is regarded as the father of photogrammetry [63,64]. He published a well-known book [65] in which he depicted his research on methods and instruments for the compilation of topographic maps. The book's completeness is demonstrated by the fact that the principles that he first laid down over 100 years ago are still in use today [66].

In 1922, the Heyde firm built an autocarthograph [67] that was constructed by Hugershoff, enabling terrain mapping from arbitrarily directed photographic images for the first time. Camera features became significant criteria in photogrammetry. Development of camera lenses with large coverage angles was one of the aims of photogrammetry research during that period. During the last two decades of the twentieth century, photogrammetry has experienced very significant changes caused by advances in optics, electronics, imaging and capture technologies, which make possible the next logical development, the digital photogrammetric workstation. Though the basic principles of photogrammetry have not changed, the operational environment has changed significantly over the past two decades. Semi-automated and automated tools continue to be developed that lighten the burden of feature extraction [68].

Copyright (C) Phanikrishna Thota 2003 


\section{Chapter 3 : TEST ARTICLE FABRICATION PROCESS: MOUNTING AND PATTERNING}

\subsection{Introduction}

Many ultra-lightweight structures are coated with aluminum or gold for special applications such as solar sails, sunshades, optical mirrors etc. Control of the surface shape is vital in such applications. It is the tracking of such metallized thin films that are considered in this thesis. Since such metallized films are optically smooth, surface treatment is required to enable the surface measurements. This chapter presents the procedure used for test article fabrication desired which is a two-step process, mounting the thin film and fabricating the pattern.

\subsection{Mounting of the Thin Film}

Ultra-lightweight thin films are so flexible that they have to be rigidly supported before they can be studied experimentally. A copper ring typically used as a gasket in vacuum applications was used for supporting the thin films in this thesis. Herein, the size of the initial test articles was defined by the size of the vacuum chamber for pattern fabrication.

\subsubsection{Procedure}

Preparation of the thin-film mirrors used to demonstrate in-plane measurement techniques comprised the initial stage of this work. The following materials were used to prepare the thin-film mirrors:

- Copper rings with inner and outer diameters of 2.011 inches and 2.425 inches, respectively, and thickness $2 \mathrm{~mm}$.

- Optical flat, 4-inch diameter and $14 \lambda$ surface flatness

- Silicone adhesive

- thin films, 2-mil thick polyester film coated on one or both sides with an $0.2 \mu \mathrm{m}$ layer of aluminum and 5 mil polyimide film coated on one side with an $0.15 \mu \mathrm{m}$ layer of gold

- Adhesive tape 
- Weights

Figure 3-1 shows the four primary components. The copper ring served as a stiff frame for attachment of the thin film. A layer of RTV - 118 silicone adhesive was applied to one face of the copper ring. The shiny face of the metallized polymer was pressed against the smooth surface of the optical flat, and temporarily affixed to the flat using adhesive tape. This maintained the tautness of the film, and resulted in a flat, mirror-like surface. The adhesivecoated side of the copper ring was then pressed against the dull side of the metallized polymer, and compressed under a 2-1b weight for 24 hours, which allowed a strong bond to form. Once a state of complete cure was reached, the weight and the tape were removed and the mirror was trimmed along the outside perimeter of the copper ring. Figure 3-2 shows an example of a cured sample prior to removal of the adhesive tape and excess film. Figure 3-3 shows completed assemblies fabricated using the double-sided aluminized polyester film (left); gold-coated polyimide film (center); and single-sided aluminized polyester film (right). Overall, fifteen mirrors were prepared.

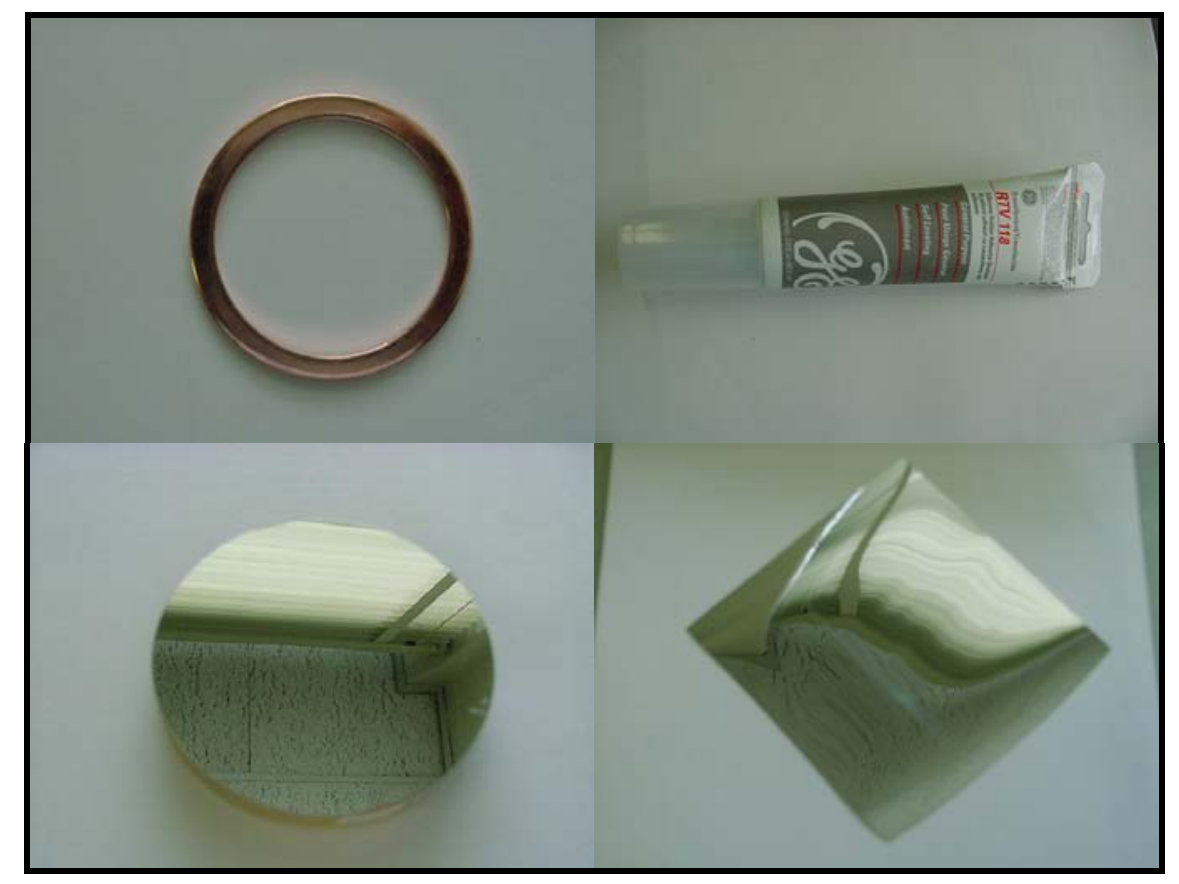

Figure 3-1: Materials used in the test article fabrication: Copper ring (top-left), silicone adhesive (top-right), optical flat (bottom-left), thin film (bottom-right) 


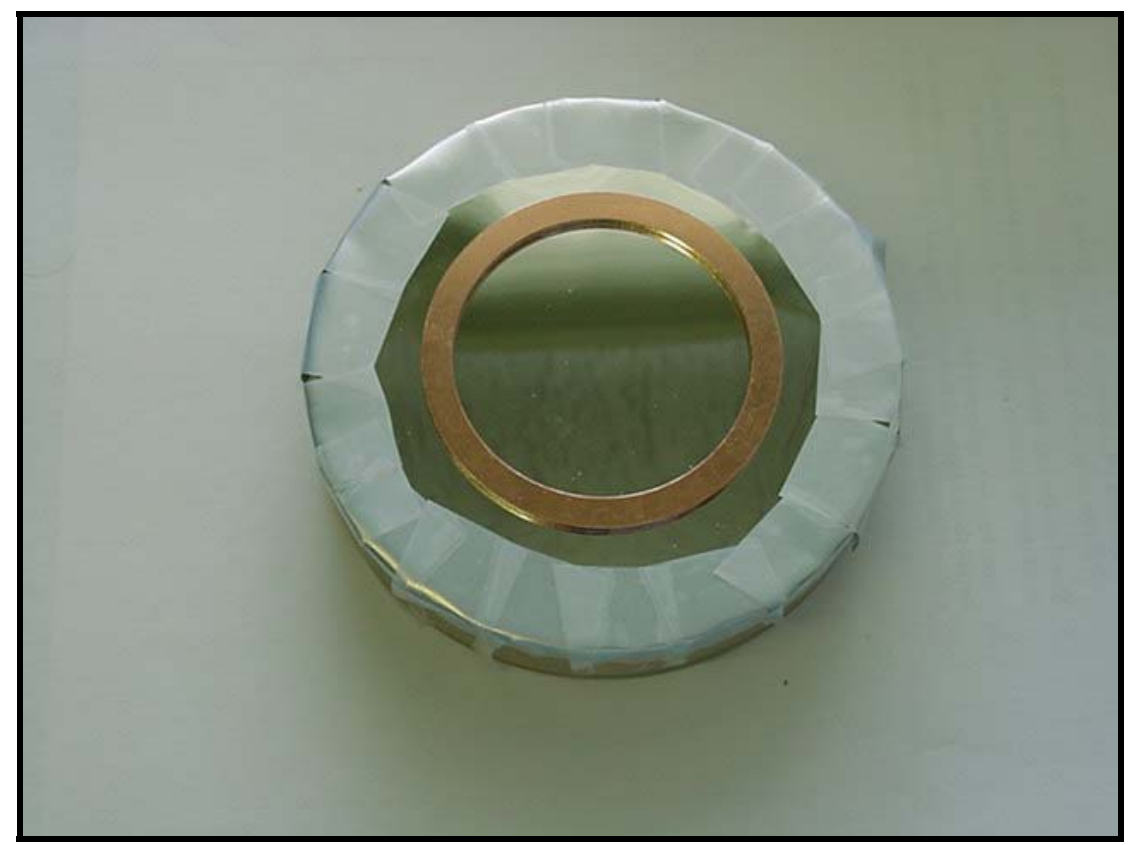

Figure 3-2: Cured sample prior to removal of adhesive tape
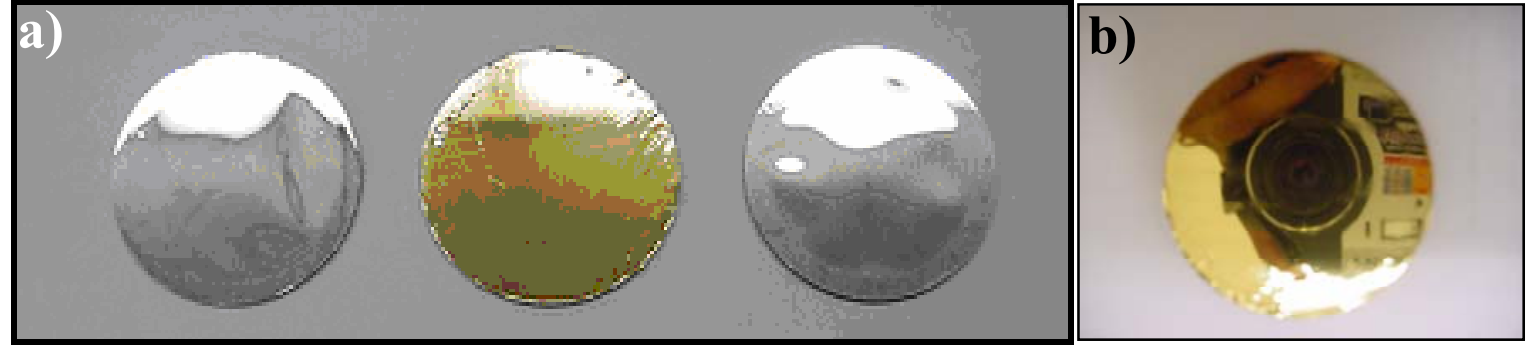

Figure 3-3: a) Two-sided polyester (left); polyimide (center); single-sided polyester (right) and b) with humidity control showing the reflected camera image

\subsubsection{Environmental Effects}

An important aspect to be considered in dealing with thin films is the effect of humidity and temperature on their surface. The thin-film mirrors used in this study were found to be much more susceptible to humidity than to temperature. In Figure 3-3a, the mirrors are noticeably not flat as a result of humidity. Storage in a sealed, airtight container containing desiccant packets allowed excess moisture to be absorbed. We found that these conditions were sufficient to maintain the flatness of the mirror surface as seen in Figure 3-3b. The effects of atmospheric conditions such as temperature and humidity on the mechanical and electrical properties were 
discussed in several textbooks [69]. From experience handling them, it was concluded that the optimal storage conditions for thin films such as these range between $45-50 \% \mathrm{RH}$ and $15^{\circ}-20^{\circ} \mathrm{C}$.

\subsection{Design and Fabrication of the Pattern}

In order to allow in-plane components of displacement to be resolved, an appropriate tracking pattern had to be designed, and then etched into the surface of the thin-film mirror. Important factors that were considered for pattern design include the effect of pattern geometry on the precision of displacement resolution, the ease of pattern fabrication and the effect of the pattern on the dynamic response of the test article.

\subsubsection{Excimer Lasers}

The acronym LASER is derived from the expression "Light Amplification by Stimulated Emission of Radiation". This refers to an electromagnetic radiation anywhere in the spectrum from ultraviolet through infrared regions, with wavelengths ranging from $0.1-1000 \mu \mathrm{m}$. Laser light has properties that include monochromaticity, coherence, high brightness, high power and large energies. Lasing action depends on the lasing medium, means of excitation, the optical resonator and population inversion. Excitation devices send the electrons of the lasing medium into their quasi-stable excited energy states. The mirrors feed back the spontaneously emitted photons so that they can oscillate and be amplified by stimulated emission, thus forming the laser beam [70].

Excimer stands for EXCIted diMER, where dimer is a diatomic molecule. It emits energy in the form of photons by its transition between energy levels. The most widely used excited dimers are Argon Flouride (ArF), Krypton Flouride $(\mathrm{KrF})$, Xenon Chloride $(\mathrm{XeCl})$ and Xenon Flouride (XeF). When chemically inert or noble gases such as Argon (Ar), Krypton (Kr) or Xenon (Xe) are excited by an external energy such as an electron beam, electric charge, or photon beam, they act as alkali metals. Consequently they react with the halogens to form the excimer.

Excimer lasers have the properties of high intensity and short duration, due to the strong strength and short lifetime of excimer bonds. Usually, excimers can emit photons with up to 1 Joule in a single pulse of 15-30 ns duration. The short wavelength of excimer lasers provides the

possibility of fabricating sub-micron size patterns with high resolution [71] and makes them excellent non-contact tools for processing metals, plastics and ceramics [72]. Besides their short 
wavelengths, excimer lasers are characterized by their high photon energies and shallow penetration depths for ablating thin layers of materials without any significant damage to the bulk sample [73].

Table 3-1: Various excimer lasers and their corresponding wavelengths

\begin{tabular}{|c|c|}
\hline Excimer & Wavelength (nm) \\
\hline $\mathrm{ArCl}$ & 175 \\
\hline $\mathrm{ArF}$ & 193 \\
\hline $\mathrm{KrCl}$ & 202 \\
\hline $\mathrm{KrF}$ & 248 \\
\hline $\mathrm{XeBr}$ & 282 \\
\hline $\mathrm{XeCl}$ & 308 \\
\hline $\mathrm{XeF}$ & 351 \\
\hline
\end{tabular}

\subsubsection{Laser Ablation}

"Laser ablation is a complex phenomenon, which occurs when a laser beam strikes a solid surface and exceeds an energy threshold to cause measurable or observable damage and material removal from the exposed surface; where the rate of removal exceeds one monolayer per pulse with atom, ion or cluster yields that are superlinear functions of the electronic excitation density"[73]. The energy threshold cited above is the level of energy required to break apart solid materials by Ultraviolet (UV) photon absorption. The two major laser parameters that influence ablation are pulse duration and pulse fluence (energy density). During removal of material via laser ablation, the absorption of photons by the target surface is accompanied by conversion of electromagnetic energy into heat [74]. The phenomenon of conversion causes the melting and evaporation of a small volume of material. The amount of energy absorbed into the material depends on several aspects of the material such as surface roughness, purity, and optical properties that include reflectivity and absorption [74]. 


\subsubsection{Excimer Laser System}

The excimer laser employed in this work consists of two major subsystems. The first subsystem is a laser system comprised of a Lambda Physik Compex 102 excimer laser, a vacuum pump and a gas cylinder. The second subsystem is a beam delivery system. The laser system is also equipped with a motion control system and a sample-processing chamber. Figure 3-4 and Figure 3-5 respectively show a schematic representation and a photograph of the laser system employed in this work.

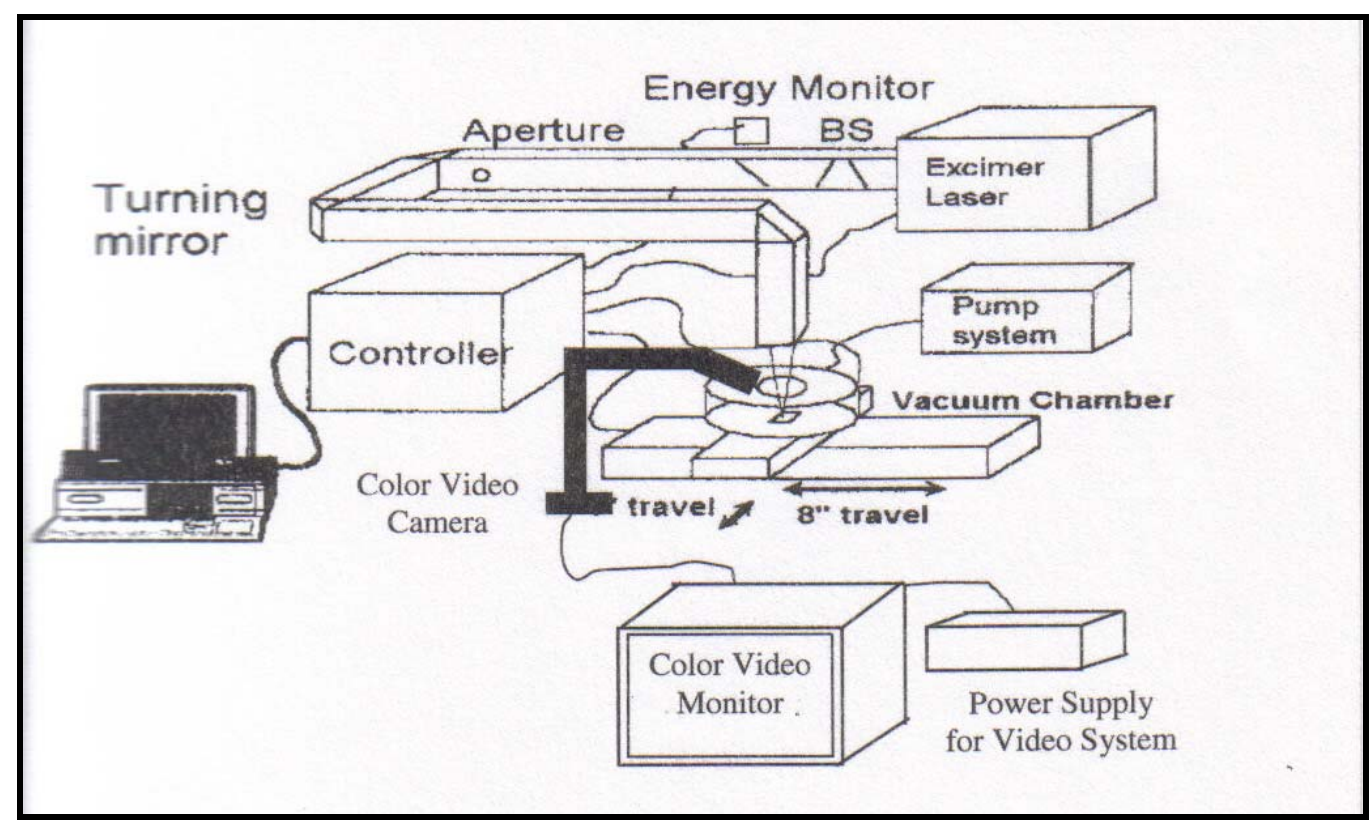

Figure 3-4: Schematic of the excimer laser system. 


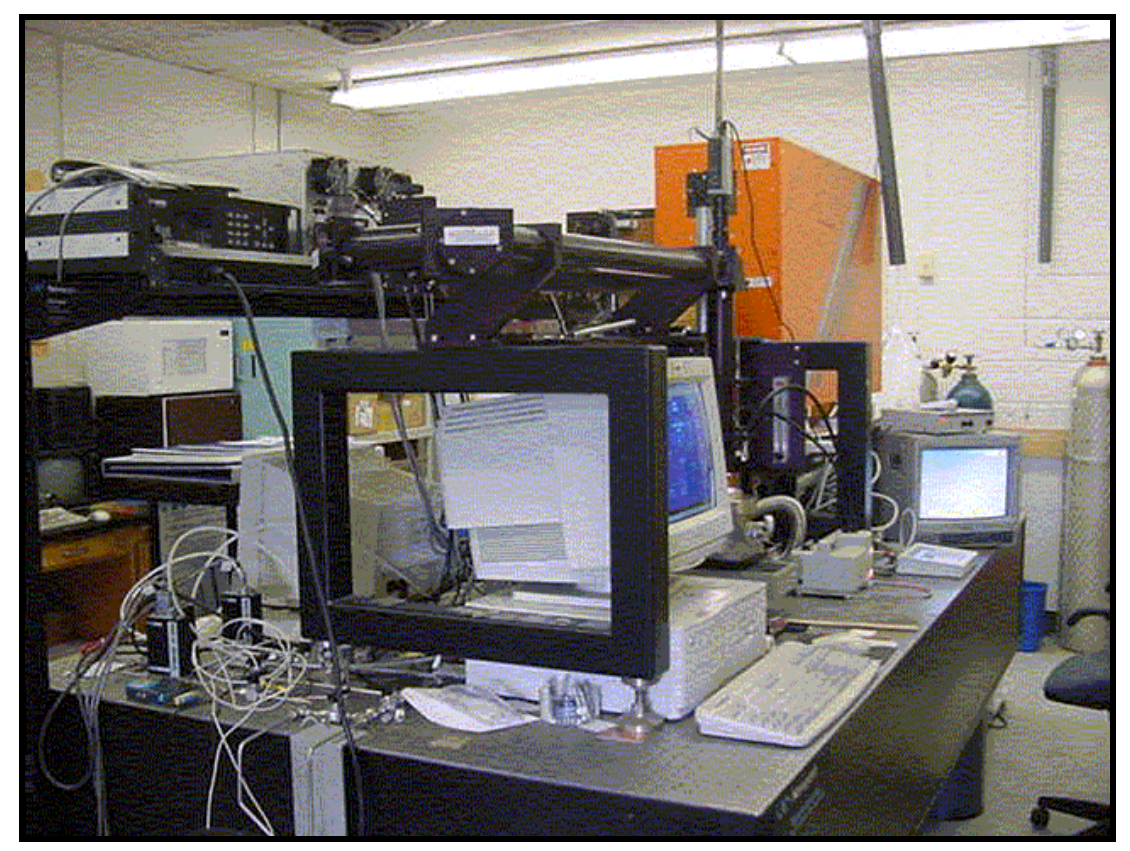

Figure 3-5: Excimer laser and associated instrumentation

\subsubsection{Laser Generation System}

The Lambda Physik Compex 102 excimer laser is set up for a $\mathrm{KrF}$ mode to operate at 248 $\mathrm{nm}$ wavelength, with a maximum possible repetition rate of $20 \mathrm{~Hz}$ set via the control panel. The electric discharge produced by the high voltage power supply is sufficient to excite the krypton atoms, which further excite the fluorine atoms, and thus forms an excited dimer.

\subsubsection{Optical Delivery System}

The laser beam is brought to its final destination by the beam delivery system shown schematically in Figure 3-6. A Zero Offset Variable Attenuator (ZOVA), designed by Resonetics, Inc. consists of two excimer laser beam attenuator optical elements that move simultaneously to impart a known attenuation to the beam. The angle between the two optical elements is controlled in order to regulate the intensity of the beam. Decreasing the angle between the optical elements causes an increase in beam reflection, resulting in a corresponding diminished transmission. 


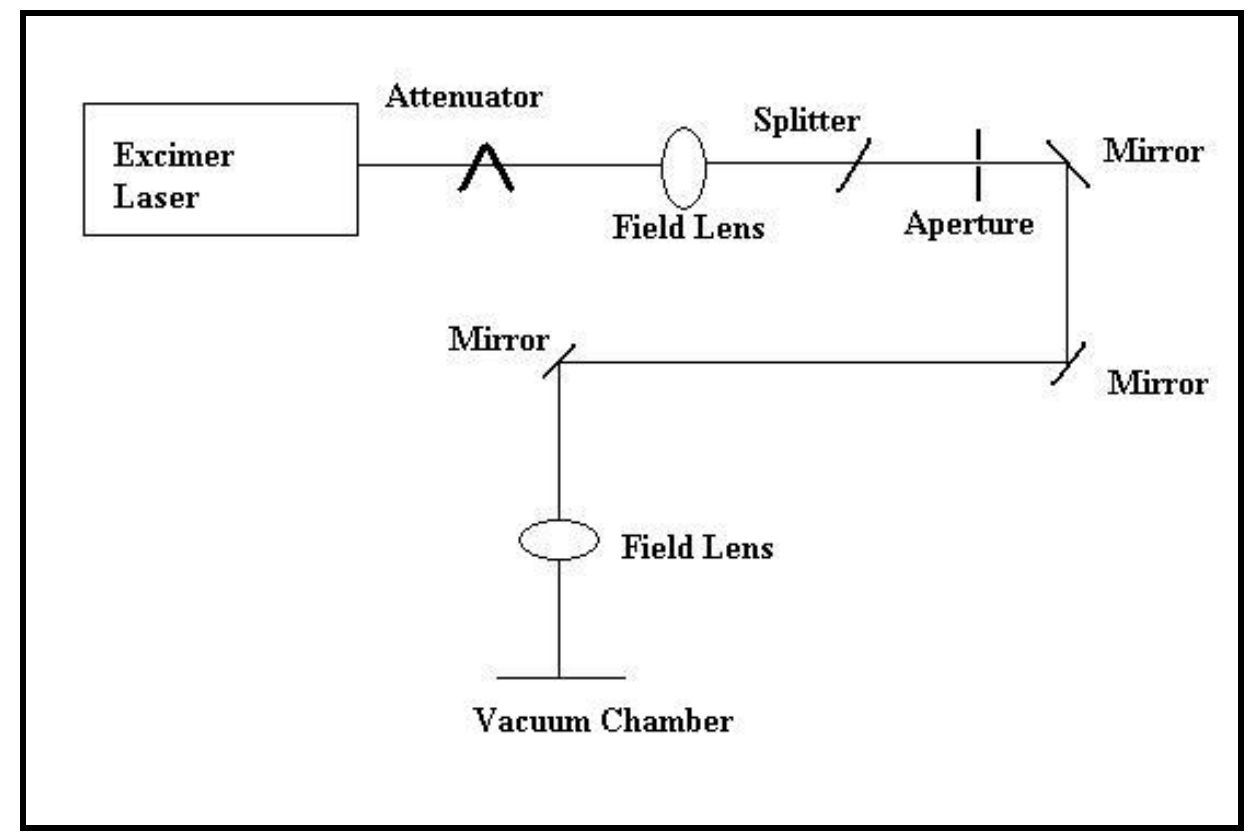

Figure 3-6: Schematic of the beam delivery system

A field lens is mounted in the path of the laser to reduce the beam divergence. A beam splitter optical element is set at an angle of $45^{\circ}$ in the path of the beam, to achieve a $60 \%$ transmission. A holder is present to support apertures of various sizes to enable different spot sizes to be generated through near field imaging. Three turning mirrors are present to direct the beam towards the processing site.

The laser beam reaches the sample-processing site through a quartz window that is $95 \%$ transparent to ultraviolet light. The process site is located so that the sample is exposed to the focused laser beam. In this case the sample site is located in a cylindrical vessel, which is mounted on motion stepper motor controlled X-Y stages. The accuracy of the stepper motors is $+/-1 \mu \mathrm{m}$ with a resolution of $0.5 \mu \mathrm{m}$. This motion control system provides four-axis open loop control, which provides hardware interface between the PC/486, X-Y stages, attenuator assembly and the laser system.

\subsubsection{Design of Candidate Feature Primitives}

The features fabricated on the thin-film mirrors were designed by considering many factors, such as incorporation of tracking marks used for other applications, utilizing patterns typically fabricated for radar applications, determining effects of feature size on the in-plane 
displacement resolution, and any effects of feature orientation. Table 3-2 lists the candidate feature primitives evaluated for their effectiveness at tracking in-plane motion of the film.

Table 3-2: Candidate feature primitives incorporated into the pattern fabricated on the thin-film test article.

\begin{tabular}{|c|c|c|}
\hline $\begin{array}{l}\text { Candidate } \\
\text { Feature }\end{array}$ & Feature Primitive & Feature Dimension \\
\hline 1 & 4 & Diameter $=0.9 \mathrm{~mm}$ \\
\hline 2 & $\bigoplus$ & Diameter $=1.1 \mathrm{~mm}$ \\
\hline 3 & |ח & Smallest dimension $=0.15 \mathrm{~mm}$ \\
\hline 4 & || & - \\
\hline 6 & $\Gamma$ & - \\
\hline 7 & $T$ & - \\
\hline
\end{tabular}

\subsubsection{Feature Patterning}

Fabrication of the test pattern on the surface of the test article was accomplished by placing the thin-film sample into the vacuum chamber, the movement of which was very accurately controlled by PC-driven stepper motors. The range of chamber motion is 8 " $(\mathrm{H})$ by 4 " (V). The geometry of the test pattern was specified using the laser's built-in CAD program, FAST CAD. The PC-controlled variable attenuator and the manually operated beam splitter were adjusted so that desired amount of energy was applied to the surface of the thin-film sample. The laser beam focus was adjusted using a micrometer that controls the position of the lens. The pressure inside the vacuum chamber was regulated via the mechanical pump and measured using a Pirani electronic pressure gauge. 
Primary factors affecting test pattern quality on the surface of the test article include specification of each laser parameter:

- Laser pulse energy $\left(135-180 \mathrm{~mJ} /\right.$ pulse, variable attenuator $15^{0}-60^{\circ}$, beam splitter $30 \%$ $-60 \%)$

- Aperture size $(1-0.45 \mathrm{~mm})$

- Laser beam focus ( $1-3$ on the micrometer)

- Test sample chamber gas pressure (760 and 2E-2 Torr)

- Laser pulse spacing $(0.001-0.01 \mathrm{inch})$.

A parameter study was carried out to optimize the laser settings used to produce the test pattern. For a given aperture size, the energy of the laser was varied from $135 \mathrm{~mJ} /$ pulse to 200 $\mathrm{mJ} /$ pulse in steps of $20 \mathrm{~mJ} /$ pulse. For each energy level, the focusing lens position adjustment was varied from positions 1 to 3 . A single laser pulse was triggered for each unique set of parameters tested. The effect of laser settings on beam profile at the surface of the thin film was evaluated by examining the test sample under a Zeiss Axiotech microscope. This device permits images to be magnified between 25 to 500 times, and is coupled to a CCD camera that allows objects to be viewed on a CRT or computer. Examples of how the laser parameters affected the shape of the beam as it impinged on the surface of the test article are shown in Figure 3-7 and Figure 3-8. Figure 3-7 shows the effect of increasing focus of the laser pulse, while Figure 3-8 shows the effect of increasing beam energy. In both cases, all laser parameters other than the ones varied were held at constant levels. Figure 3-9 shows the microscopic view of the aperture used to fabricate the entire pattern on the thin-film mirror. The dimensions of the rectangular aperture are also indicated on the figure. Since a rectangular pulse was obtained with a square aperture and all laser parameters set, a rectangular aperture was manufactured using the laser itself to counter this problem and obtain a square pulse. A square pulse was required to obtain equal feature resolution in horizontal and vertical directions and due to the presence of circular features. A circular aperture of diameter $0.46 \mathrm{~mm}$ was used to fabricate the required rectangular aperture. 


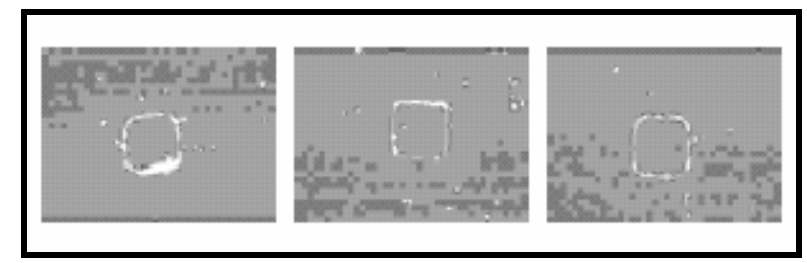

Figure 3-7: Laser pulse shape variation as a function of lens position (Left - 1, Middle - 1.25, Right - 1.5)

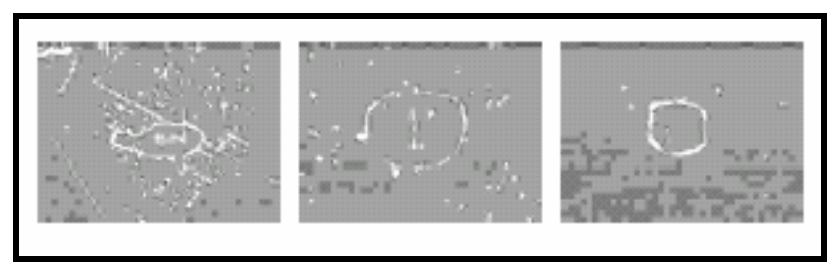

Figure 3-8: Laser pulse shape variation as a function of beam splitter transmission value for constant pulse energy of $\mathrm{mJ}$ (Left $-60 \%$, Middle $-42 \%$, Right $-30 \%$ )

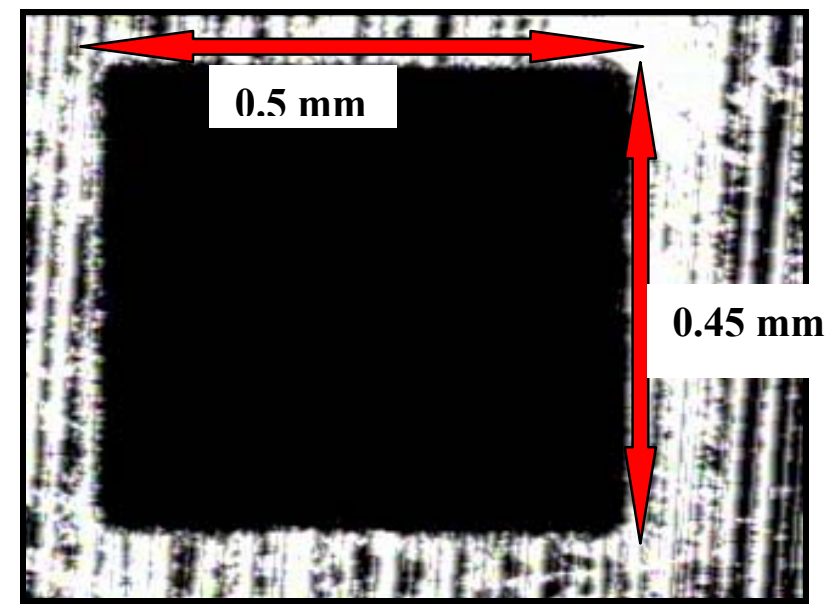

Figure 3-9: Microscopic view of the aperture through which the laser beam passed; used to fabricate the entire pattern.

A Tencor Alpha-Step 500 stylus profilometer was used to measure the surface profile of the fabricated thin film. This contact device has a 1-angstrom resolution, which is sufficient to resolve slight differences in surface profile. Laser pulse spacing refers to the distance between the pulses used for metallization removal on the surface of the thin-film mirrors. Adjustment of this parameter affects the depth of metal removal. Figure 3-10 and Figure 3-11 show the surface profile of pattern obtained by using the profilometer in the initial stages of the fabrication process. The features fabricated had a depth of about $0.1 \mu \mathrm{m}$. The depth of fabrication can be easily seen in Figure 3-10 and Figure 3-11. Reading from the figures, average feature ranged 
between $15 \times 100 \AA$ to $20 \times 100 \AA$, or $0.15 \mu \mathrm{m}$ to $0.2 \mu \mathrm{m}$. These surface profile plots confirmed removal of the metallization layer without significant penetration of the substrate.

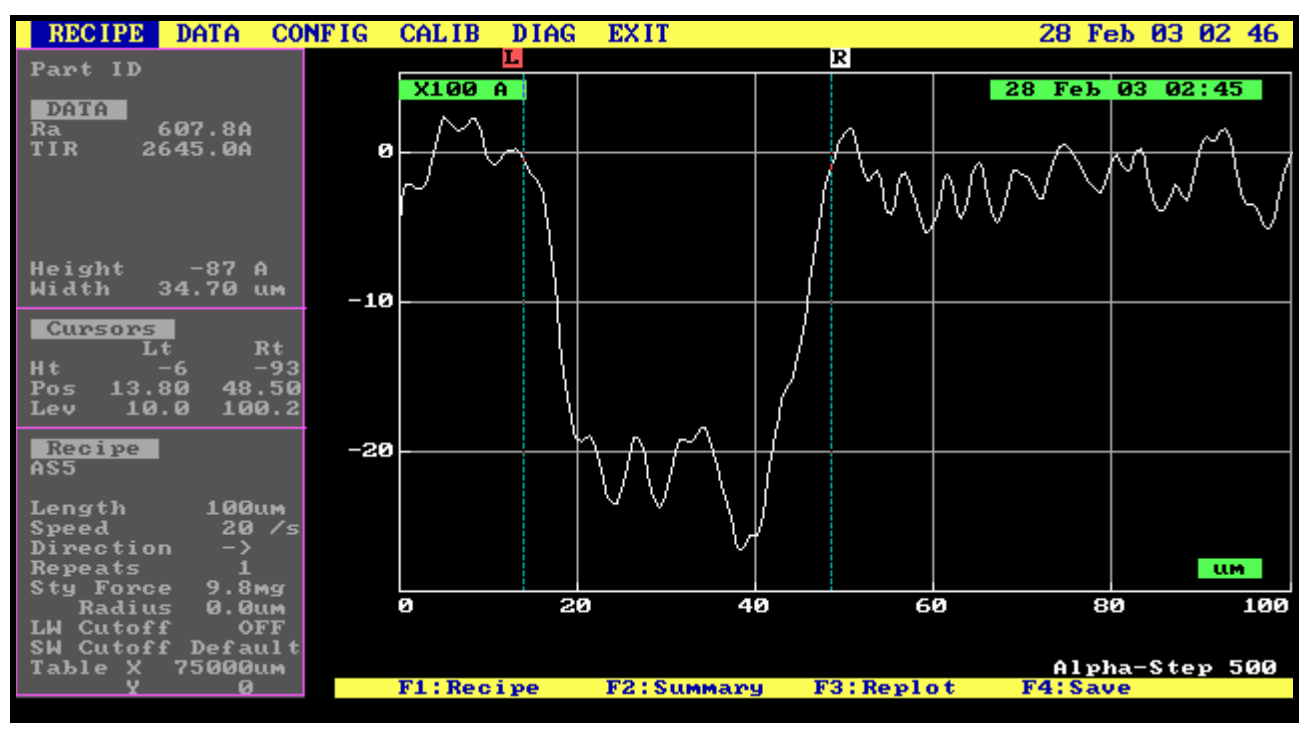

Figure 3-10: Surface profile of the pulse

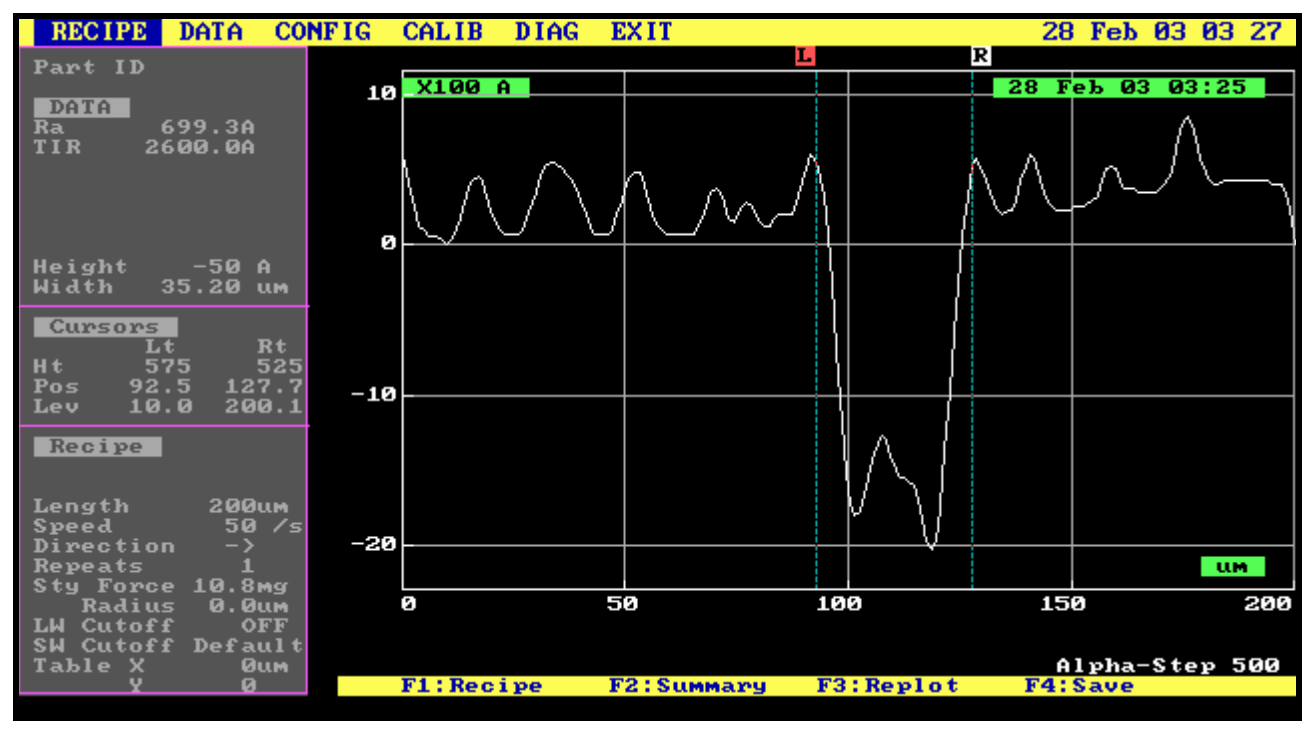

Figure 3-11: Surface profile of the pulse at another position on the pattern

After completing the test series, the settings shown in Table 3-3 were chosen for fabrication of the patterns on the Al coated mirrors. Slightly less energy (Variable attenuator- $18^{0}$ ) was used for pattern fabrication on the gold-coated films. The pulse used to fabricate the pattern on the thin-film was a square pulse with a side length of approximately $35 \mu \mathrm{m}$, which was 
obtained using the rectangular aperture mentioned previously. Hence all the features were fabricated with a width of approximately $35 \mu \mathrm{m}$. In the course of this work the gas cylinders of the excimer laser system were changed, which caused some of the optimized settings used to fabricate the patterns to vary slightly. To determine the new settings, a parameter study was once again conducted. The optimized system values for the new gas cylinders remained constant, except for the angle of variable attenuator, which changed from $30^{\circ}$ to $18^{0}$.

Figure 3-12 and Figure 3-13 show the microscopic views of the features fabricated on the thin-film mirror with the laser parameters set to the values in Table 3-3. These features are a part of the pattern fabricated on the thin-film mirror, which is shown in Figure 3-14.

Several dimensions of the fabricated features were verified using an optical microscope. The fabricated feature dimensions when measured using the optical microscope were found to be larger by about $50 \mu \mathrm{m}$ than the dimensions created in FAST CAD.

Table 3-3: Laser parameters chosen for pattern fabrication

\begin{tabular}{|l|l|}
\hline Parameter & Setting for Al- coated film \\
\hline Aperture size & $0.45 \mathrm{~mm}$ X $0.5 \mathrm{~mm}$ \\
\hline Laser Energy & $150 \mathrm{~mJ} / \mathrm{pulse}$ \\
\hline Beam Splitter & $60 \%$ \\
\hline Angle of variable attenuator & $30^{\circ}$ \\
\hline Pulse spacing & 0.0012 in \\
\hline Vacuum Chamber Pressure & $2 \times 10^{-2}$ Torr \\
\hline
\end{tabular}

The time taken to fabricate this pattern on the thin-film mirror was about 3.5 hours with a repetition rate of $10 \mathrm{~Hz}$ and about 2 hours with a repetition rate of $15 \mathrm{~Hz}$. Voltage fluctuations also had little effect on the energy of the laser and in turn on the amount of metal removed from the surface of the thin-film mirror. Insufficient laser energy resulted in a pattern with non-sharp areas along the feature edges, and left traces of metallization. 


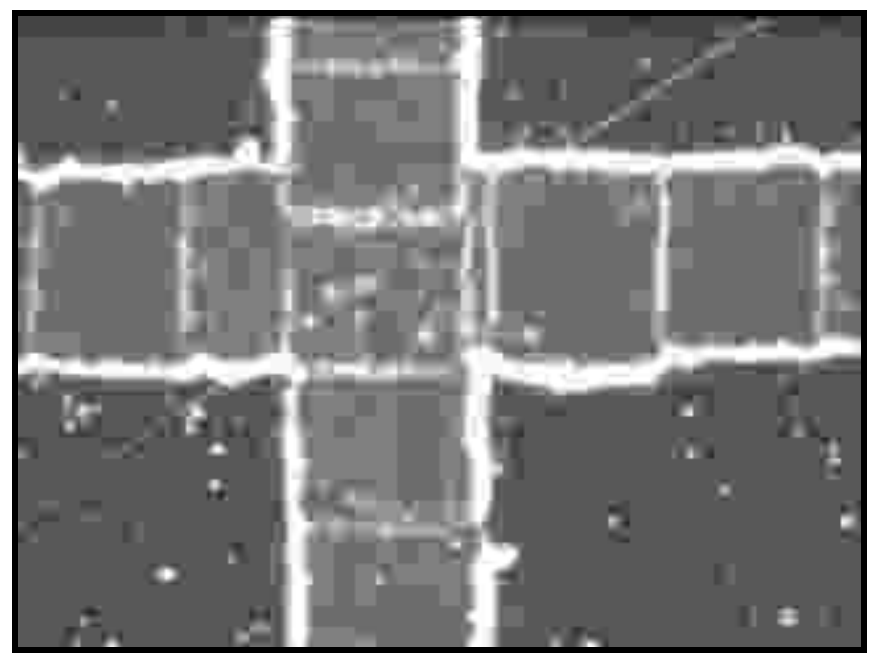

Figure 3-12: The laser pulses shown overlapping to form a cross (The rectangular shape of the pulse immediately to the left of the vertical stripe is due to overlapping of the pulses)

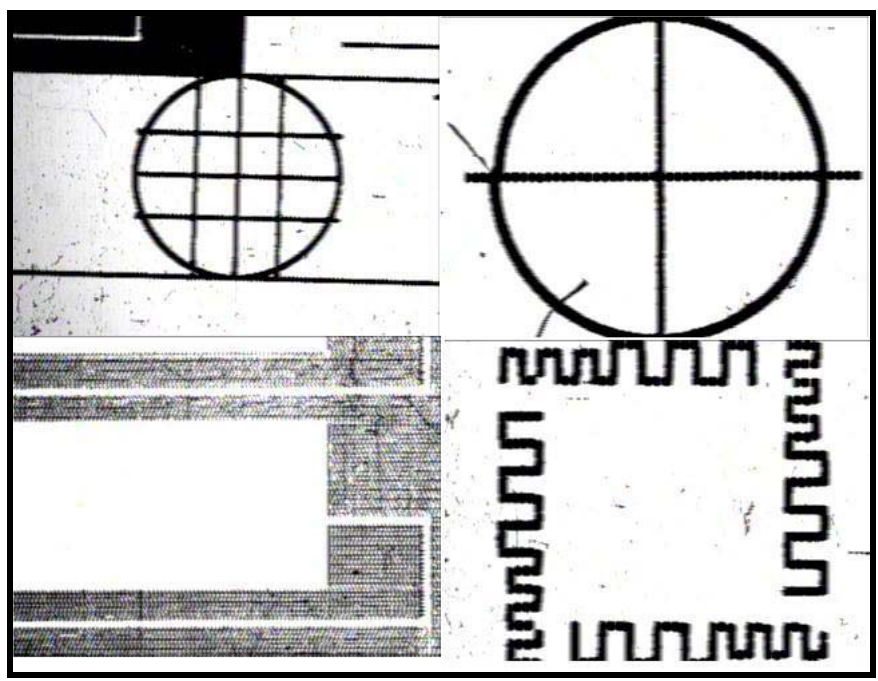

Figure 3-13: Magnified view of selected features fabricated on the thin-film mirror 


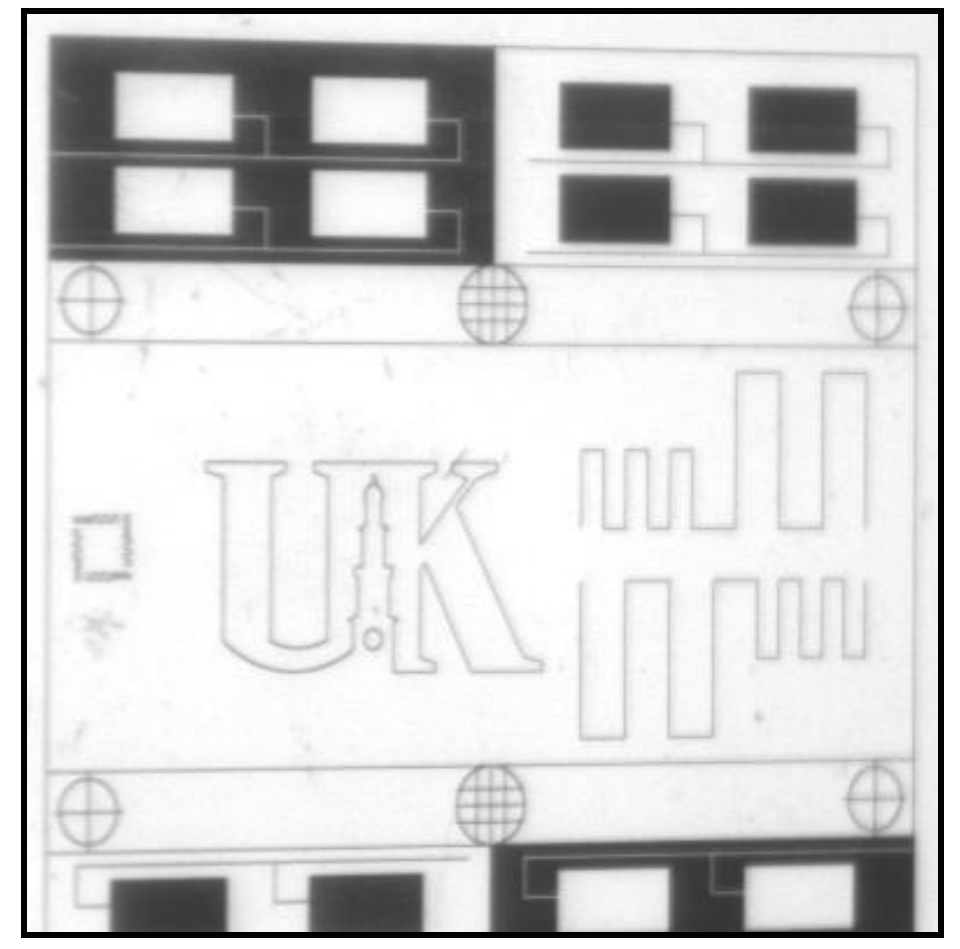

Figure 3-14: Sample test pattern comprised of geometric features listed in Table 3-2. 


\section{Chapter 4: FINITE ELEMENT ANALYSIS OF ETCHED THIN-FILM MIRRORS.}

\subsection{Introduction}

The primary concern related to using contacting sensors for surface displacement measurements in gossamer structures is that they tend to affect the dynamic response of the structure. Although it was believed that the proposed displacement targets fabricated into the surface of the thin-film mirrors would not cause a significant change in dynamic response, a Finite Element (FE) study was performed to verify this. Moreover if the computational models of the test articles are verified experimentally they can be extended to study similar full-scale structures. As any change in the dynamic characteristics of a system will affect its natural frequencies, calculating the modes of the test articles with and without the fabricated pattern is the means selected to evaluate the impact of the pattern on the dynamics of the structures. Finite element analyses of static displacement were also performed and compared to experimental measurement of test article response.

\subsection{Finite Element Models}

Five FE models were produced in ANSYS version 6.1 to quantify the effect of the fabricated patterns on the dynamics of the thin-film mirrors. Four models similar to the singlesided aluminum test articles and a more generalized, larger model were produced. Table 4-1 gives the values of the material properties used to develop the models. All of the FE models contained various aspects of the physical test article; while an exact geometric model of the test article and etched patterns was produced, it was not meshed due to problems such as aspect ratios of elements, limitations on the maximum number of degrees of freedom (DOF) available in the software, as well as the complexity of the model. ANSYS SHELL91 layered elements were used to mesh the thin-film in all the models. This element was selected because layers of different thicknesses can each be assigned unique material properties. This element is generally used to model the behavior of layered composite materials with fewer than three layers. The geometry, node locations and the coordinate system for this element are shown in Figure 4-1. Complete details of this element are described in the ANSYS Users Manual element reference guide. 
Table 4-1: Material Properties used in the Finite Element Models

\begin{tabular}{|c|c|c|c|}
\hline Material & $\begin{array}{c}\text { Young's Modulus } \\
{[\mathbf{P a}]}\end{array}$ & Density $\left[\mathbf{K g} / \mathbf{m}^{\mathbf{3}}\right]$ & Thickness \\
\hline Polymer & $2.9 \mathrm{E} 9$ & 1433 & $50 \mu \mathrm{m}$ \\
\hline Aluminum & $7 \mathrm{E} 10$ & 2700 & $0.1 \mu \mathrm{m}$ \\
\hline Copper & $1.28 \mathrm{E} 11$ & 8960 & $2 \mathrm{~mm}$ \\
\hline
\end{tabular}

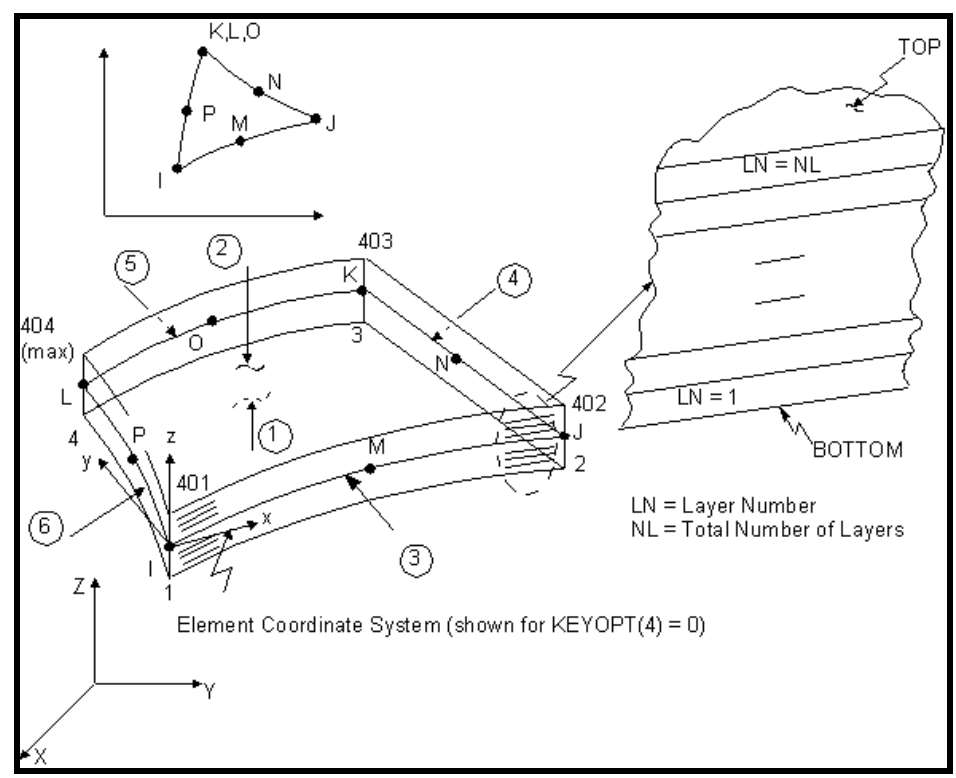

Figure 4-1: Geometry, node locations and coordinate system for SHELL91 elements [75].

\subsubsection{Model 1}

Model 1 contained almost all aspects of the completed test article, including the patterned thin-film mirror and the copper support ring. Pattern features that consisted of $35 \mu \mathrm{m}$ lines were excluded from the model because their dimensions were significantly smaller than those of any other feature of the model. Contact elements were used to model the interface between the thin film and the ring, and SOLID45 elements were used to mesh the 2-mm thick copper ring. Typical handbook values for the material properties of the aluminum metallization, polyester film, and copper were used for the model [76,77] as summarized in Table 4-1. The areas 
containing the fabricated pattern were meshed assuming a metallization thickness of $1 \times 10^{-12} \mathrm{~m}$, as setting this value to zero to represent complete material removal would cause computational divergence. Regions where the top layer metallization was not removed used a layer thickness of $1 \times 10^{-7} \mathrm{~m}$. The base polymer thickness, which was $5 \times 10^{-5} \mathrm{~m}$, remained constant throughout the entire model. Mesh density was increased in areas where intermediate sized triangular elements were used to interface between the quadrilateral elements used to represent the central features and the large triangular elements present in the outer regions of the film (Figure 4-2). This model was constrained around the outer edge via constraints applied to the copper ring.

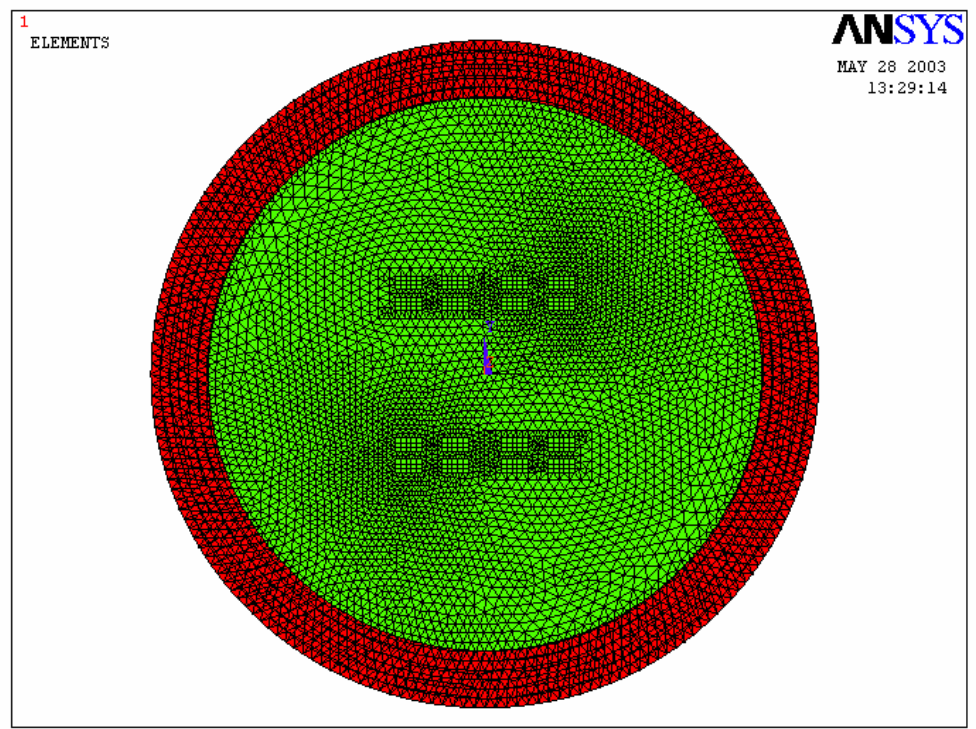

Figure 4-2: Meshed Model 1

\subsubsection{Model 2}

Model 2 was the same as Model 1, but omitted the fabricated pattern shown in the central region of Figure 4-2. This model was produced to evaluate the modes of the thin-film mirror without the pattern, but did include the copper support ring in the modal analysis. Since there was metallization all over the thin-film mirror, the entire thin-film mirror was meshed with double-layered SHELL91 elements with the thicknesses of the base polymer and metallization set at $50 \mu \mathrm{m}$ and $0.1 \mu \mathrm{m}$ respectively. Figure 4-3 shows the meshed Model 2 along with the copper support ring. 


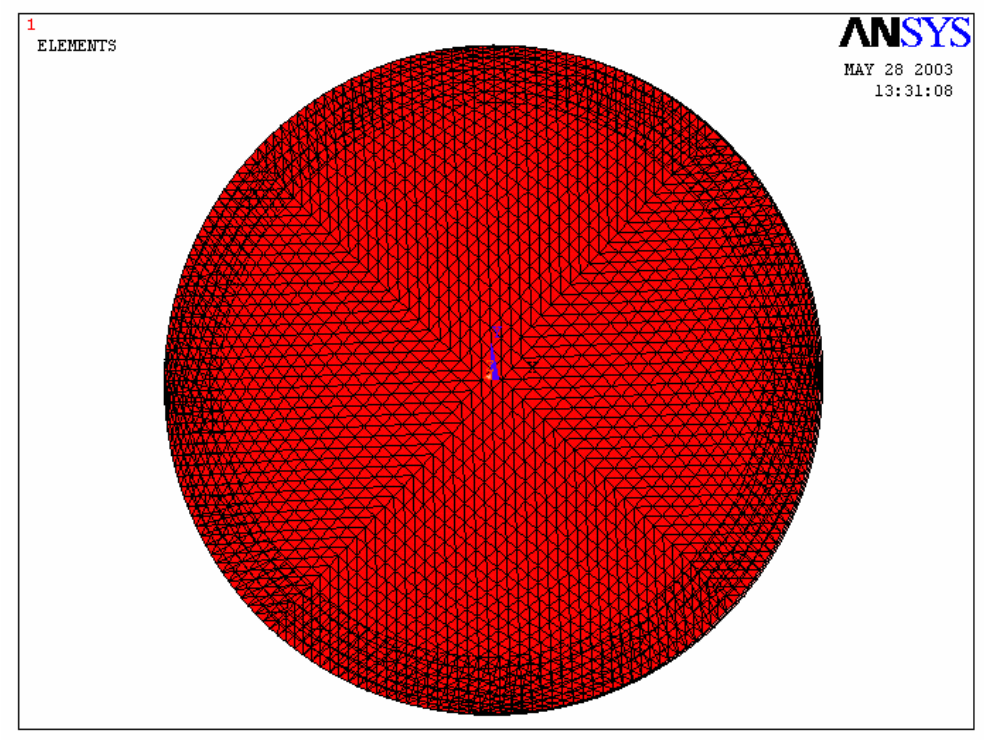

Figure 4-3: Meshed Model 2

\subsubsection{Model 3}

Model 3 contained the patterned film, but excluded the copper ring. In this model, the diameter of the thin film was set as the inner diameter of the copper ring, 2.011 inches. This model was produced to reduce the complexity caused by copper ring and the contact elements without sacrificing accuracy in calculating the fundamental frequency of the thin-film mirrors. The features fabricated on the test article with widths of $35 \mu \mathrm{m}$ or less were again not modeled here due to the meshing complexities. The model was constrained in all DOF along its circumference. Figure 4-4 shows meshed Model 3, incorporating the etched pattern. As was the case for Model 1, this model is not meshed with uniform triangular elements because of the detailed pattern in the center of the model.

\subsubsection{Model 4}

Model 4 was the simplest of all models used to simulate the response of the thin-film mirror, and is shown in Figure 4-5. It consisted of polymer film uniformly coated with an aluminum metallization layer. Its diameter was set equal to the inner diameter of the copper ring. This model was also constrained in all DOF along its circumference. Since there was no pattern in this model, the circular symmetry of the mesh can be clearly seen in the Figure 4-5. 


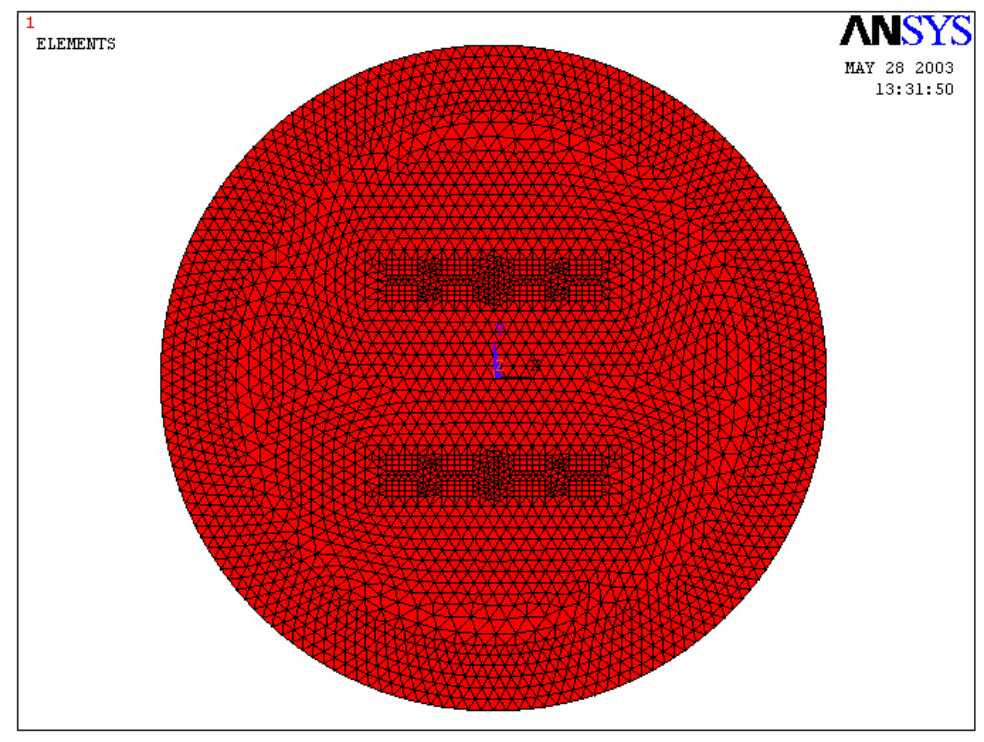

Figure 4-4: Meshed Model 3

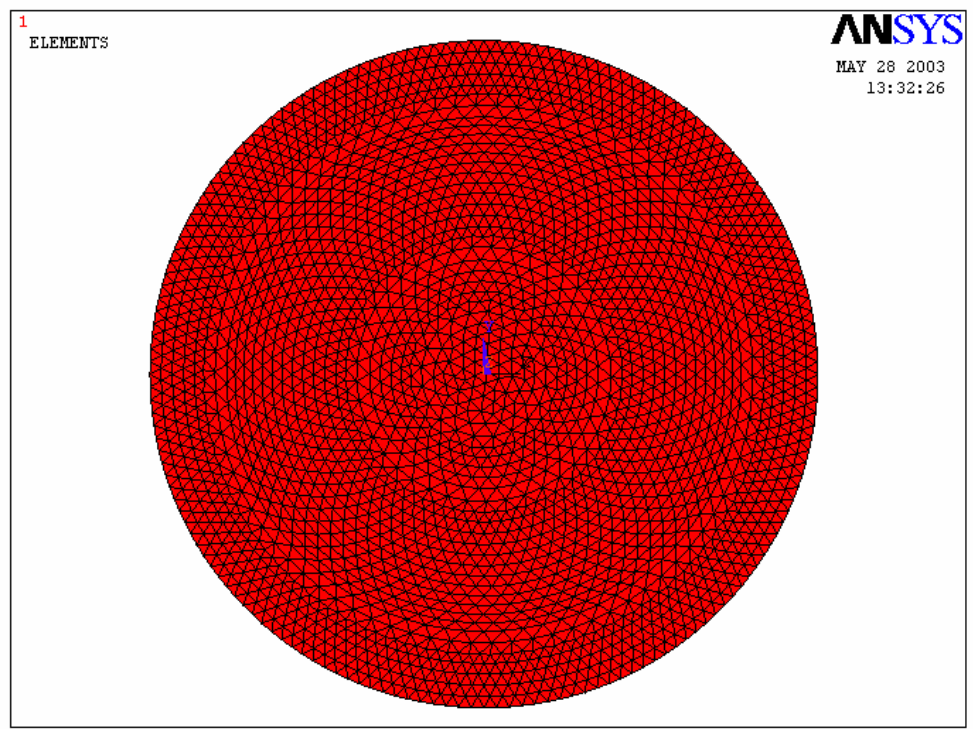

Figure 4-5: Meshed Model 4

\subsubsection{Model 5}

To further generalize the effect of metallization area on the modal response of polymer film, and to study the relationship between total film area and relative area of metallization, finite element models of $0.5 \mathrm{~m}$ diameter thin films were created. Initially a $25 \mu \mathrm{m}$ thick polymer film with $1 \mu \mathrm{m}$ thick aluminum coating was considered. Modal analysis was performed on the model, and natural frequencies were compared for many different pattern geometries. The meshed 
model is shown in Figure 4-6 and a zoomed-in view of the densely-meshed area used to simulate various patterning schemes is shown in Figure 4-7. An area of 1 sq. in was divided into 1600 (40 $x$ 40) small squares so that metallization removal from these small squares could be simulated by setting their top layer thickness to near zero. Starting from complete metallization on the thinfilm, complete non-metallization on the 1 sq. in area was achieved gradually in 40 steps by removing the metallization from the small squares. Modal analysis was performed at each step and the results were documented. Figure 4-7 shows the gradual increase in the mesh size from a small square of $1 / 40$ in size to a considerably larger triangle. This was done to avoid any sudden changes in mesh sizes in the model.

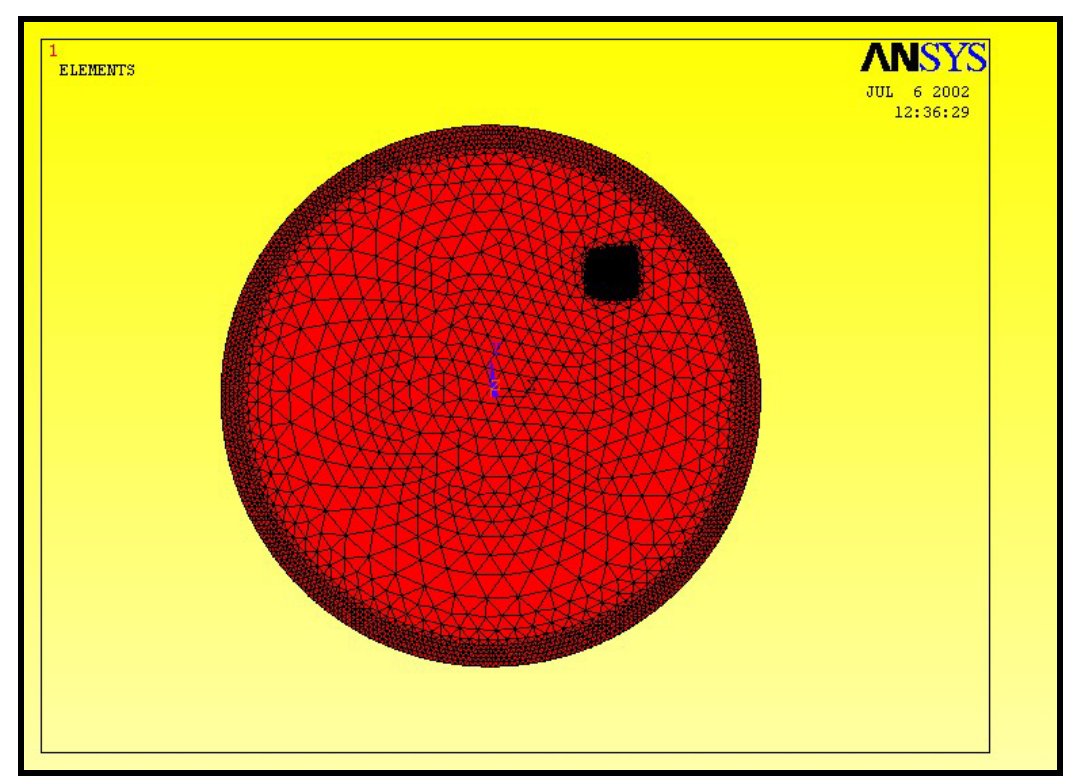

Figure 4-6: Meshed Model 5 


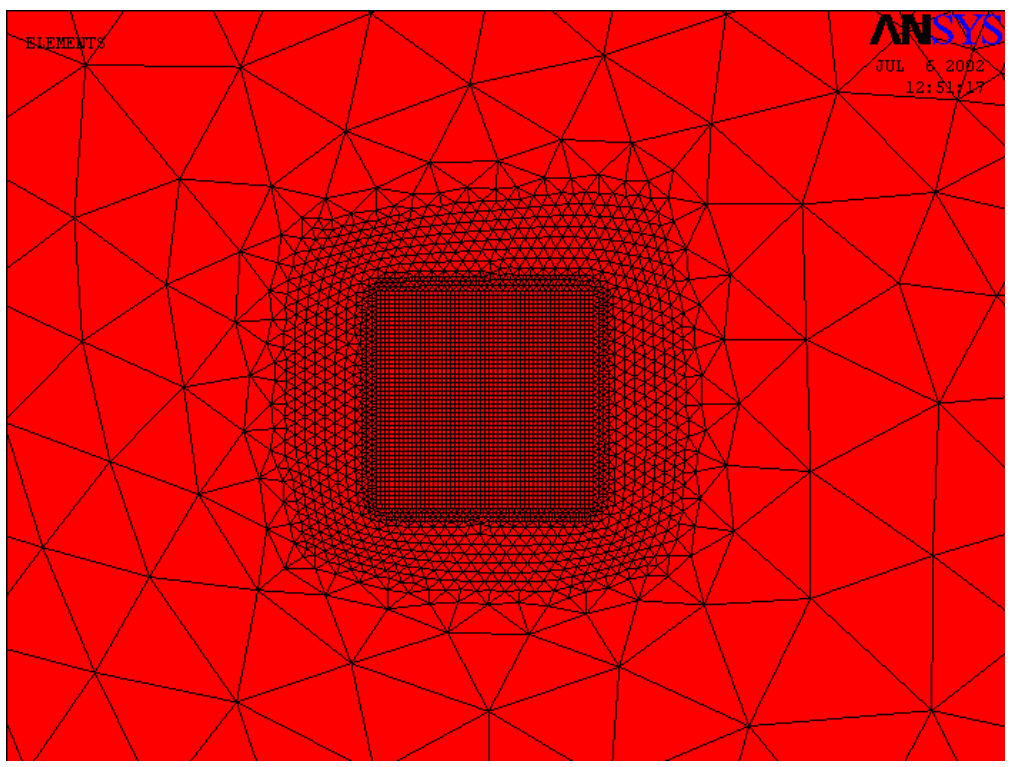

Figure 4-7: Zoomed in Model 5 (Area of non-metallization)

\subsubsection{Unmeshed Model 6}

This model incorporated all features of the test article, and could not be meshed due to the required element aspect ratios and the largely diversified element sizes. All aspects of the test articles, including the coppering, the complete pattern and the thin-film polymer were included in this model. Since this model could not be meshed with the limitation on the available version of the software, it was decided to create simpler models (i.e. 1 - 4) whose behavior would still accurately represent the dynamics of the test article. Figure 4-8 shows the unmeshed Model 6 and Figure 4-9 shows an example of the $35 \mu \mathrm{m}$ features that were not considered in the meshed Models 1-4. 


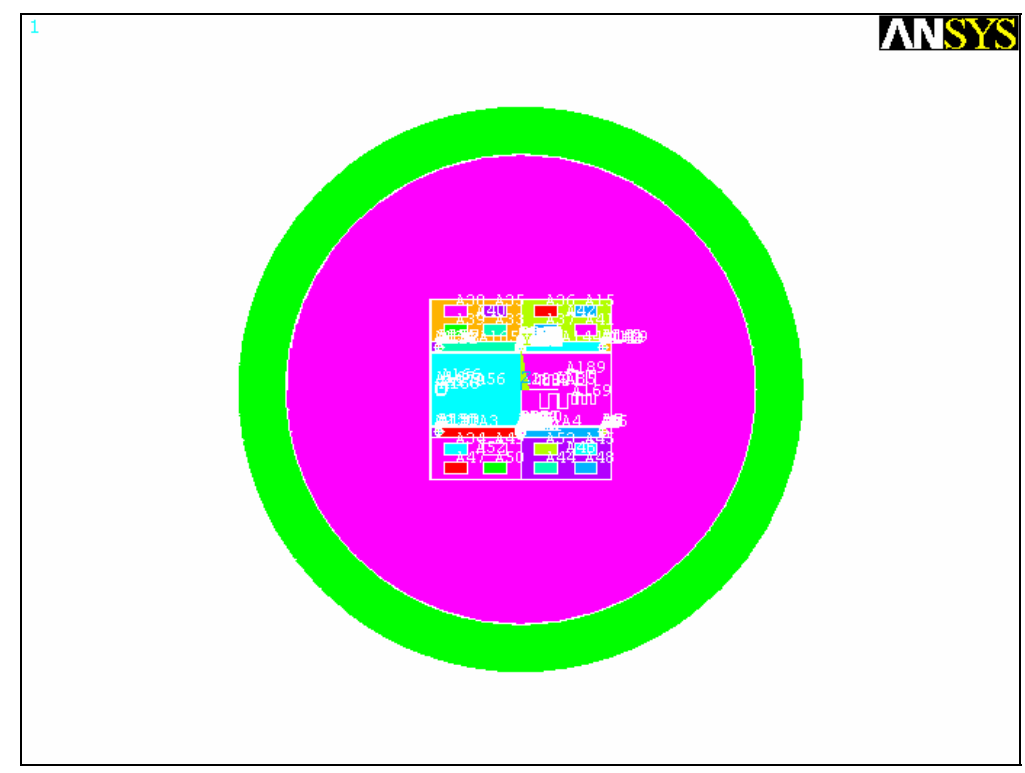

Figure 4-8: Unmeshed Model 6

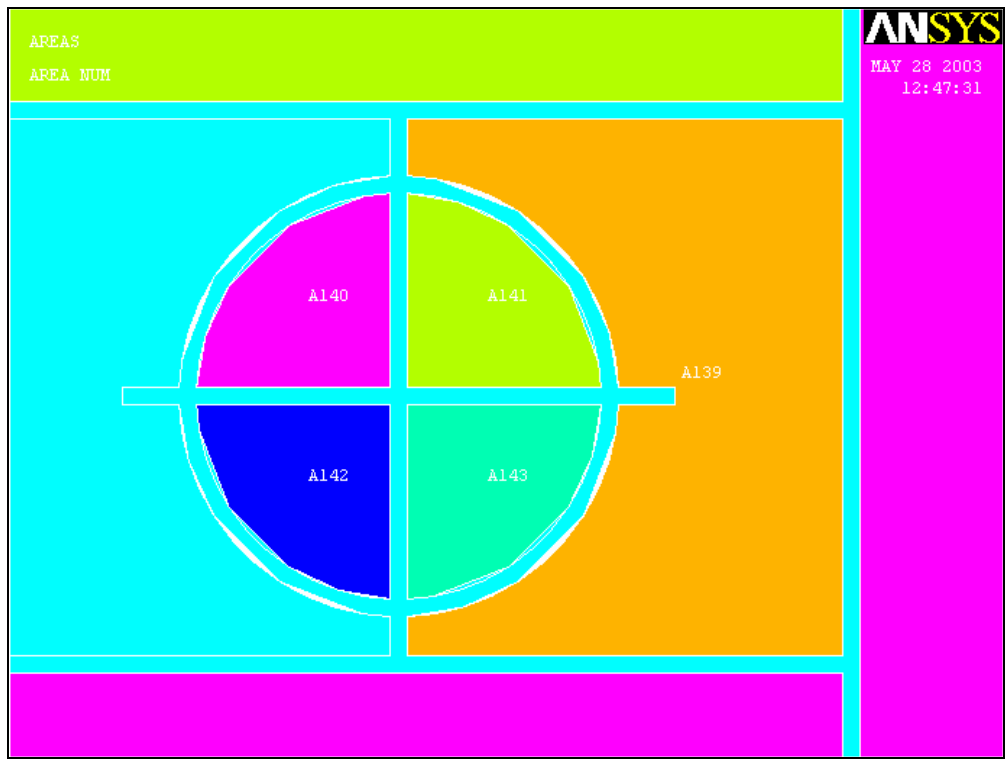

Figure 4-9: Features with $35 \mu \mathrm{m}$ width.

\subsection{Modal Analysis of the Finite Element Models}

In order to evaluate the affect of the fabricated pattern on the thin-film mirrors, modal analysis of the different models of the thin-film mirrors described in the previous section was simulated. The fundamental frequencies computed were recorded from the analysis and the mode shapes were studied. 


\subsubsection{Modal Analysis of the Test Article Finite Element Models}

Models 1 through 4 were the models of the test article used for the modal analysis, and each included various aspects of the physical test article. Modal analysis of these models was performed and the first ten modes and their frequencies were extracted using Block Lanczos method. Table 4-2 shows the different frequencies obtained using Models 1-4. Note that the fundamental frequency for the test articles is approximately $60 \mathrm{~Hz}$. The standard frame rate for video is 32 fames per second. Therefore, for dynamic experiments a frame rate of at least $120 \mathrm{~Hz}$ or a larger diameter test article is needed to avoid undersampling.

The difference between the fundamental frequency obtained for Models 2 and 4 was $0.102 \mathrm{~Hz}$, which was less than $0.5 \%$ of the nominal fundamental frequency. This indicated that the effect of modeling the copper ring is negligible on the dynamics of the thin-film mirrors. Modal analysis of the copper ring alone was performed and the fundamental frequency was found to be about $800 \mathrm{~Hz}$, which was much higher that the fundamental frequency of the test articles. Models 1 and 3 have a fundamental frequency difference of $1.123 \mathrm{~Hz}$, which was about $1.8 \%$ of the fundamental frequency. This, again, indicates that the copper ring does not affect the dynamics of the test articles significantly. The difference in fundamental frequencies between Models 3 and 4 was $0.359 \mathrm{~Hz}$, or $0.6 \%$ of the nominal frequency, and the difference in the fundamental frequencies between Models 1 and 2 was $1.78 \mathrm{~Hz}$, or $2.29 \%$. Therefore, the effect of the pattern on the dynamic response was small.

It is evident from the table that the second and third frequencies are equal when there is complete metallization on the thin-film mirror, but with the pattern, these frequencies split. Except for the frequency of the $2^{\text {nd }}$ mode of Model 1 (effect of pattern is $6.62 \%$ ), the effect of pattern on the $2^{\text {nd }}$ and $3^{\text {rd }}$ mode frequencies $(<1.1 \%)$ is smaller than on the fundamental frequency.

Modal analysis was also performed after replacing the metallization on Model 3 and the results obtained were same as that of Model 4. This indicates that the difference in the natural frequencies is not due to mesh differences but because of the pattern in the form of nonmetallization on the surface of the thin-film mirror. 
Table 4-2: Fundamental Frequencies of Different Models

\begin{tabular}{|c|c|c|c|c|c|}
\hline $\begin{array}{c}\text { Model } \\
\text { No }\end{array}$ & $\begin{array}{c}\text { Pattern } \\
\text { Present }\end{array}$ & $\begin{array}{c}\text { Copper } \\
\text { Ring } \\
\text { Included }\end{array}$ & $\begin{array}{c}\text { Fundamental } \\
\text { Frequency }\end{array}$ & $\begin{array}{c}\text { Second / Third } \\
\text { Frequencies }\end{array}$ & $\begin{array}{c}\text { \% Of Metal } \\
\text { Layer Retained }\end{array}$ \\
\hline 1 & Yes & Yes & $58.798 \mathrm{~Hz}$ & $117 / 126.6 \mathrm{~Hz}$ & 95.5 \\
\hline 2 & No & Yes & $60.178 \mathrm{~Hz}$ & $125.3 / 125.3 \mathrm{~Hz}$ & 100 \\
\hline 3 & Yes & No & $59.921 \mathrm{~Hz}$ & $123.9 / 124.8 \mathrm{~Hz}$ & 95.5 \\
\hline 4 & No & No & $60.28 \mathrm{~Hz}$ & $125.5 / 125.5 \mathrm{~Hz}$ & 100 \\
\hline
\end{tabular}

\subsubsection{Modal Analysis of the $\mathbf{0 . 5} \mathrm{m}$ Diameter Thin-film}

Modal analysis of the $0.5 \mathrm{~m}$ diameter thin-film (Model 5) was performed for all 40 levels of metallization removal. The first six modes calculated were rigid translation modes; the $7^{\text {th }}$ and $8^{\text {th }}$ modes were a pair of orthogonal, identical-frequency modes when there was complete metallization. The rigid body modes occurred because the model was unconstrained. As the nonmetallized area was increased in the simulation, the pair modes separated i.e. the frequency of the $7^{\text {th }}$ mode decreased while the frequency of the $8^{\text {th }}$ increased. Figure 4-10 shows the difference in the pair mode frequencies as a function of the non-metallized area. From the results shown in Figure 4-10, it is evident that the effect of pattern area on the dynamic response of thin films of this size may be as low as $0.01 \%$. The nominal natural frequency of this system, corresponding to $100 \%$ metallization, was calculated by ANSYS to be $0.22 \mathrm{~Hz}$. Although the fundamental frequency of the thin film did not change significantly for these larger samples, the nodal diameter, about which the mode shape was symmetric, tended to reorient itself as the pattern orientation changed, to maintain symmetry. 


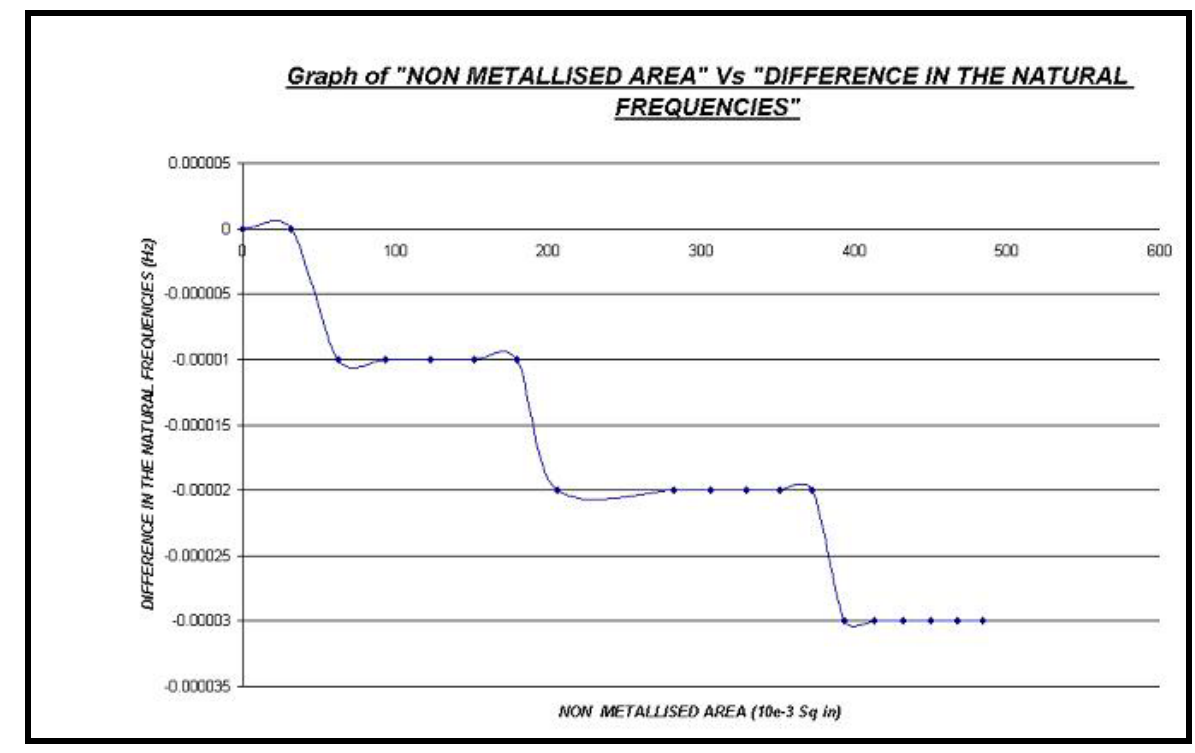

Figure 4-10: Variation in the Fundamental Frequency as a Function of the Metallized Area.

\subsection{Static Analysis of the Test Article Finite Element Models}

Model 3 was used for the performance of the static simulations since the results of the modal analysis show that the four models do not vary significantly in their dynamic characteristics. Model 3 was chosen because it contains the pattern details but not the copper ring. It was believed that the large differences in material properties between the copper and the polymer film could cause computational difficulties for the large-displacement static analysis described herein.

In this analysis, the force was applied at the center of the model over a circular area in the form of pressure on the elements. This was done by selecting elements in the required circular area and entering the total pressure value into a dialogue box. Here, the value of pressure is not divided by the number of elements, unlike the case of force in which the total force is divided by the number of nodes in the area of application. The model was constrained in all the DOF at the circumference. Though material nonlinearities should be included in the model to obtain more accurate results, linear isotropic, elastic material properties were used for simplicity. However because of the large displacements calculated, static analysis geometric nonlinearities were included in the model. The required computational step size was defined as $0.1 \mathrm{sec}$; the time at the end of the load step was $1 \mathrm{sec}$. The analysis was carried out for 5 different loads. A few lines were chosen on the film and their change in length for different load steps was recorded from the 
analysis. This approach was taken because the lines always stay in the plane of the thin-film mirror. Hence they would be useful to quantify the in-plane displacement of the thin-film mirror for the given load acting on a circular area at the center of the mirror.

\subsubsection{Results}

Large displacement static analyses were performed for five different load steps on the thin-film mirror. In Figure 4-11 and Figure 4-12, x- and y-components of displacement of the thin-film mirror are shown with the application of $1.962 \mathrm{~N}$ and $9.81 \mathrm{~N}$ force, respectively. These results are for $50-\mu \mathrm{m}$ thick polyimide film with $0.1-\mu \mathrm{m}$ thick aluminum coating. It can be observed that the $\mathrm{x}$ - and $\mathrm{y}$-displacements are symmetric about a line that is inclined to the vertical. This is because the pattern fabricated on the thin-film mirror is symmetric about that line. The maximum in-plane displacement was found near the areas of the pattern where maximum metallization was removed. This occurred near the rectangular features in the pattern that resembled features for radar applications. Maximum in-plane displacement was also found on a very small area at the center of the thin-film mirror where the force was applied. 

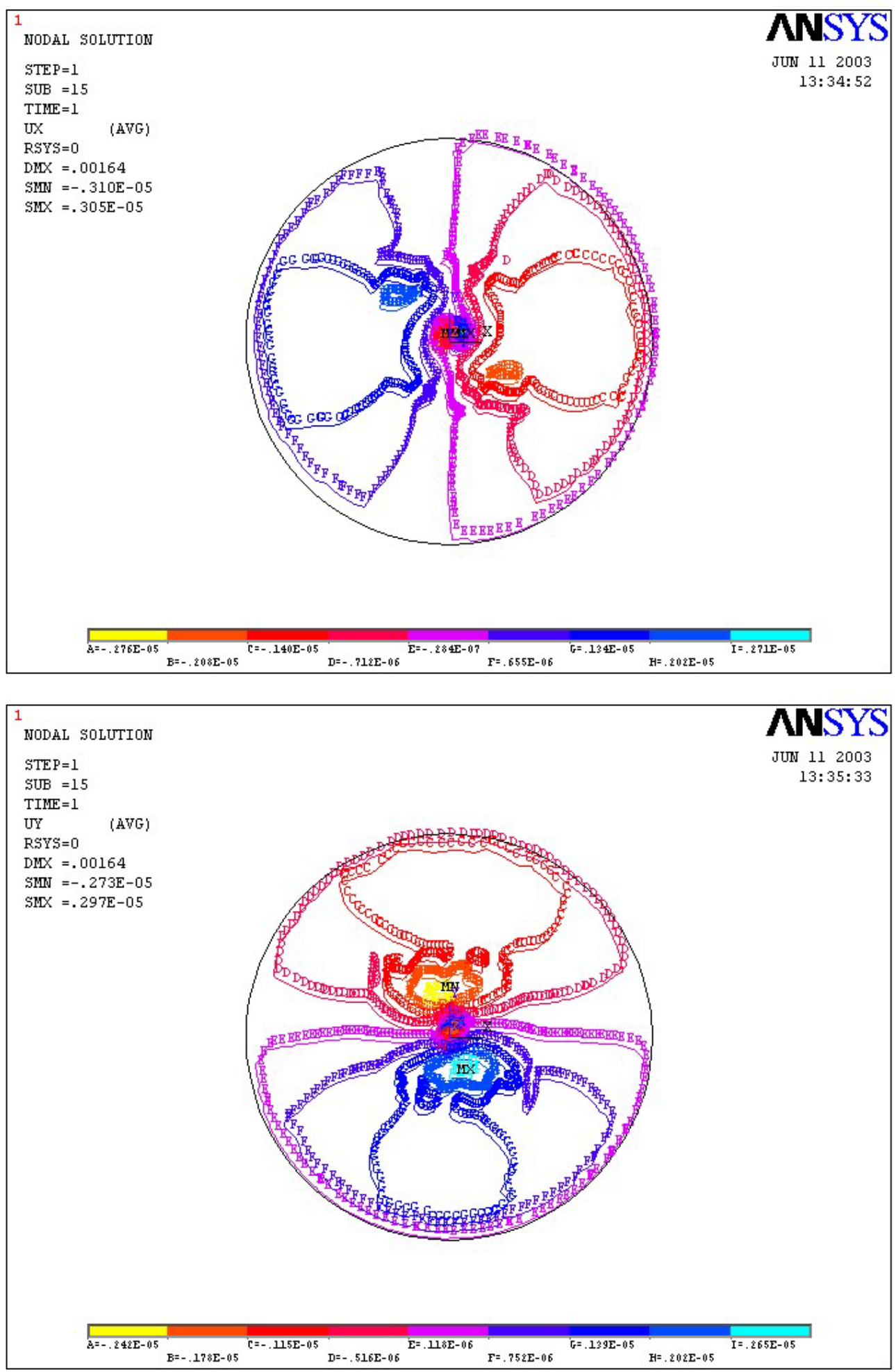

Figure 4-11: In-plane displacement ( $\mathrm{m}$ ) in the $\mathrm{x}$-direction (Top) and y-direction (Bottom) for the application of $1.962 \mathrm{~N}$ force at the center. 

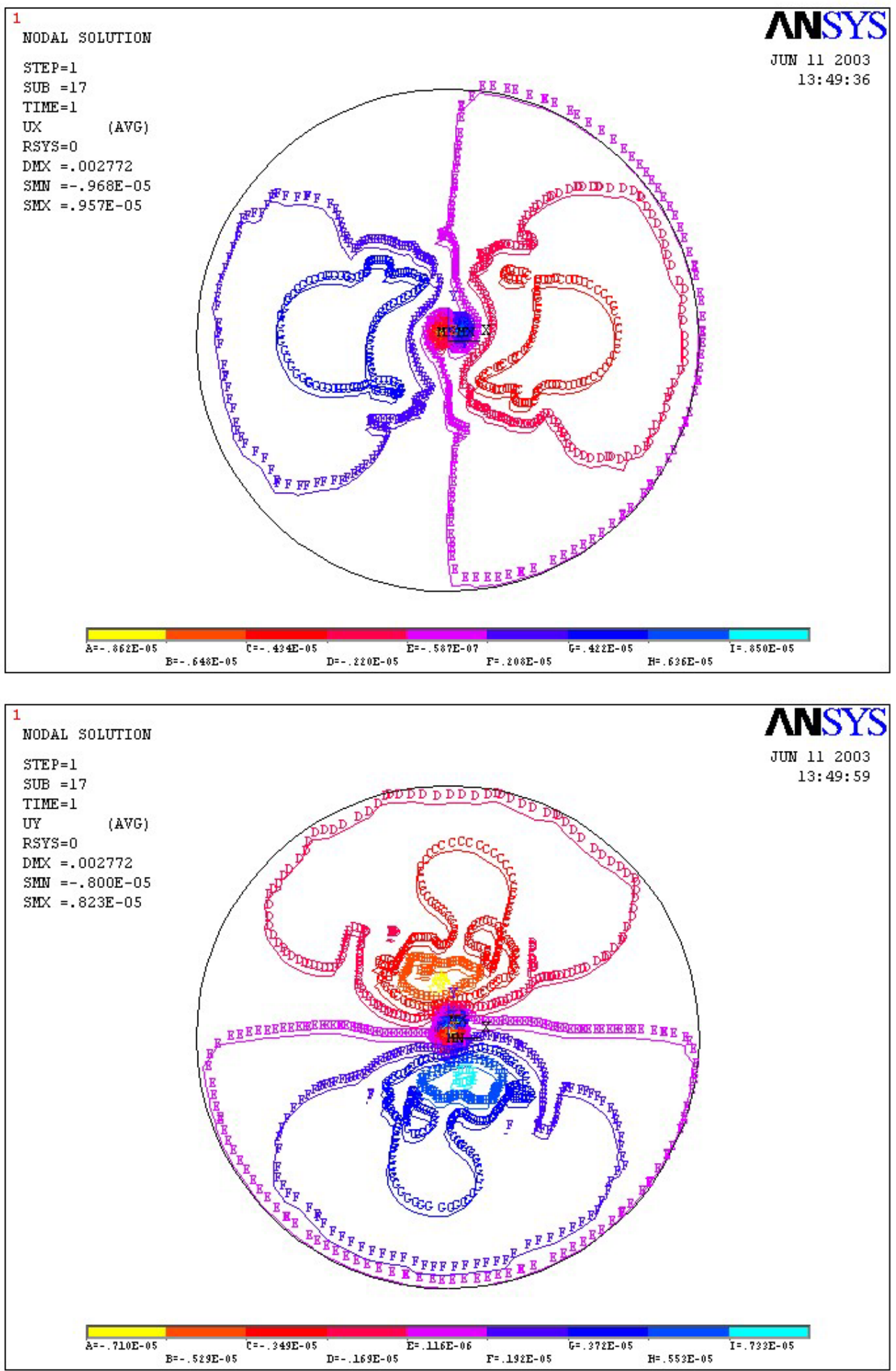

Figure 4-12: In-plane displacement (m) in x-direction (Top) and y-direction (Bottom) for the application of $9.81 \mathrm{~N}$ force at the center of the mirror 
Figure 4-13 shows the out-of-plane displacement of the thin-film mirror with the application of 9.81 force at the center of the mirror. It is evident from the figure that the out-ofplane displacement is symmetric about any diameter on the thin-film mirror. It is maximum at the center of the mirror and minimum at the circumference because it is constrained in all DOF.

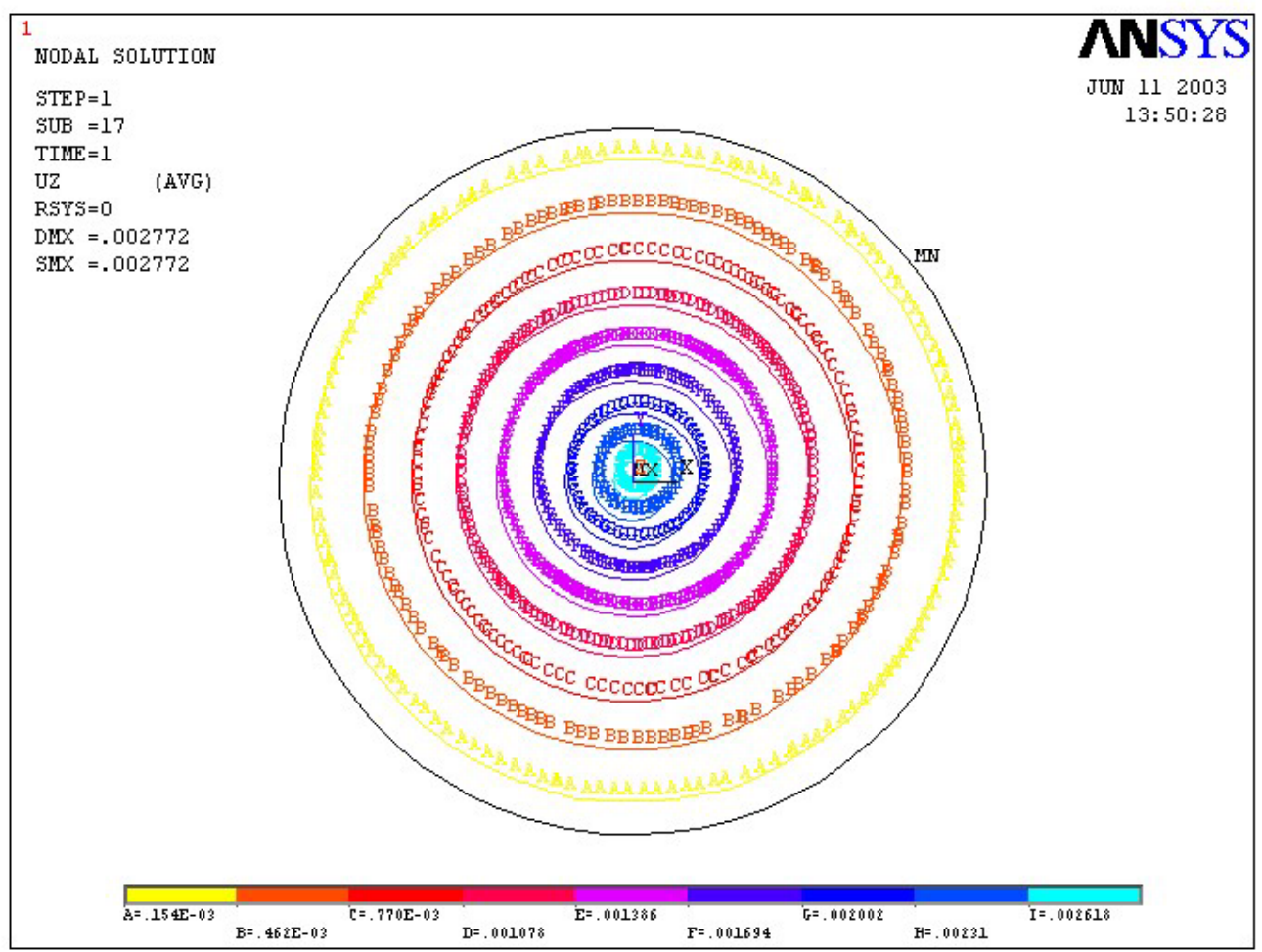

Figure 4-13: Out-of-plane displacement (m) of the thin-film mirror with the application of 9.81 $\mathrm{N}$ of force.

Table 4-3 presents the results from the finite element analyses performed on the thin-film mirror by applying different force levels at its center. The values tabulated are the maximum displacements at any location on the thin-film mirror in the corresponding direction for a particular force level. In Chapter 6, these results are compared to results from the experiments conducted on the thin-film mirror. Table 4-4 gives a comparison of results for variation in the thickness of the polymer. This was performed to study the effect of variation of parameters on the in-plane displacement of thin-film mirrors. 
Table 4-3: FE results summarizing maximum displacement components of the thin-film mirror

\begin{tabular}{|c|c|c|c|}
\hline $\begin{array}{c}\text { Force } \\
\text { Applied }\end{array}$ & X-Max $(\boldsymbol{\mu m})$ & Y-Max $(\boldsymbol{\mu m})$ & $\begin{array}{c}\text { Z-Max } \\
(\mathbf{m m})\end{array}$ \\
\hline $1.962 \mathrm{~N}$ & 2.71 & 2.65 & 1.549 \\
\hline $3.924 \mathrm{~N}$ & 4.26 & 3.88 & 1.911 \\
\hline $5.886 \mathrm{~N}$ & 5.89 & 5.07 & 2.194 \\
\hline $7.848 \mathrm{~N}$ & 7.16 & 5.98 & 2.401 \\
\hline $9.81 \mathrm{~N}$ & 8.5 & 7.33 & 2.618 \\
\hline
\end{tabular}

Table 4-4: Comparison of the results for variation in the thickness of the polymer.

\begin{tabular}{|c|c|c|c|c|c|c|}
\hline & \multicolumn{2}{|c|}{$\mathbf{X}-\operatorname{Max}(\boldsymbol{\mu m})$} & \multicolumn{2}{c|}{$Y-M a x(\mu \mathrm{m})$} & \multicolumn{2}{c|}{$Z$-Max (mm) } \\
\hline $\begin{array}{c}\text { Applied } \\
\text { Force }\end{array}$ & $50 \mu \mathrm{m}$ & $45 \mu \mathrm{m}$ & $50 \mu \mathrm{m}$ & $45 \mu \mathrm{m}$ & $50 \mu \mathrm{m}$ & $45 \mu \mathrm{m}$ \\
\hline $1.962 \mathrm{~N}$ & 2.71 & 2.93 & 2.65 & 2.87 & 1.549 & 1.591 \\
\hline $9.81 \mathrm{~N}$ & 8.5 & 9.64 & 7.33 & 8.81 & 2.618 & 2.785 \\
\hline
\end{tabular}

Copyright $(\odot$ Phanikrishna Thota 2003 


\section{Chapter 5 : PHOTOGRAMMETRY}

\subsection{Photogrammetry}

Photogrammetry is the science of measuring three-dimensional objects using multiple photographs of a single scene, taken from a number of different vantage points. This method was first used for the creation of topological maps from aerial surveys [78]. More recently, it was found that using photogrammetry, very accurate measurements of smaller objects could be performed, provided that suitable photographs of the object are obtained [79]. This technique, known as close-range photogrammetry, uses digital imaging sensors [80] and computer data analysis to generate three-dimensional solid models of the object under study. It has been shown to be a flexible and robust technology with demonstrated potential for measuring ultralightweight structures [81].

\subsubsection{Concepts in Digital Photogrammetry}

\subsubsection{Digital Image Geometry}

A digital image consists of a regular array of picture elements, usually referred as pixels. Each pixel of the digital image is a direct or indirect sample of a continuous incident light distribution. A digital sensor, known as a charge-coupled device (CCD) samples the incident light directly. Pixels are typically square or rectangular, and the ratio between pixel width and pixel height is known as the aspect ratio. Consequently, a square pixel has an aspect ratio of one.

One of the parameters related to pixel size is the ground sample distance (GSD), which is the projection of the pixel size onto the ground plane (Figure 5-1). Even though the GSD is similar to sampling resolution, GSD and resolution are not synonymous. The total resolution of a digital image is determined by both the sensor geometry and by factors external to the sensor such as atmospheric conditions, platform motion, etc. The actual resolution may be as good as that indicated by the GSD, however in reality it is often lower [78]. 


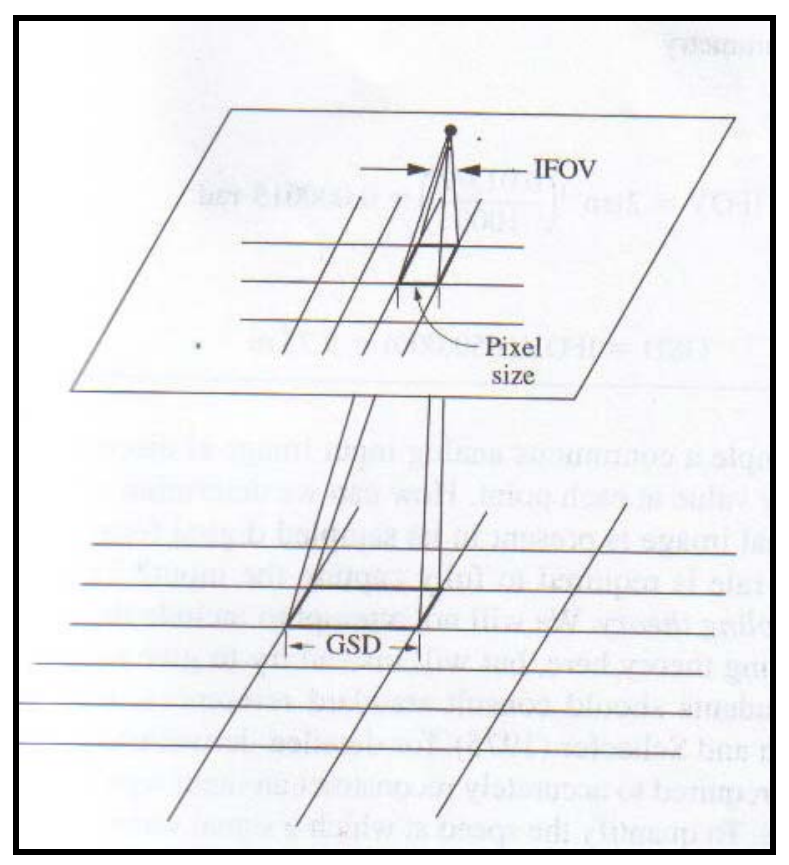

Figure 5-1: Ground Sample Distance [68]

\subsubsection{Target Measurement.}

Photogrammetry is often concerned with locating and measuring predefined targets. Techniques for locating these targets differ, and depend upon the shape and size of the targets, as well as their gray-level variation from the background material. If the target gray levels are sufficiently different from the background, simple thresholding can be used to identify pixels corresponding to the target. However, due to image noise, the target boundary or "edge" may appear uneven within the image. To overcome this problem, the centroid of a target can be calculated and used for measurement instead of the edges. This technique, however, requires large, well-defined targets such as circles, squares, triangles, etc. The centroid is usually calculated by first fitting a function to edge pixel coordinates and then finding the centroid of the resulting function. The location of the target centroid can therefore be calculated with sub-pixel precision [78].

\subsubsection{Photogrammetry Process}

PhotoModeler Pro Version 4.0 was used to determine the three-dimensional coordinates of pattern features on the surface of the test articles using digital photographs taken from a variety of orientations. Using PhotoModeler Pro v4.0, producing three-dimensional surface 
maps is an eight-step process. Each step is described in detail below, including specifics for the experiments of this thesis:

\section{Establish measurement objectives and accuracy requirements}

An important consideration in developing ground-test objectives is a good understanding of mission requirements and important design issues so they can be adequately validated. A good estimate of the required measurement accuracy for each photogrammetry project is also important, avoiding under- and over-estimating requirements. Under-estimation can lead to unacceptable or unreliable measurements while over-estimation can waste time and resources because the cost-versus-accuracy relationship is one of diminishing returns at higher accuracy levels [62].

The points to be identified using photogrammetry correspond to the targets whose 3D coordinates are identified in the real world scene. The camera must be chosen according to the accuracy requirements. Increasing camera resolution allows more accurate location of the target points. Depending upon the quantity and distribution of the targeted locations, camera positions are determined so that each target appears in at least two photographs. Scale bars can be used as reference points that allow absolute scale to be determined. This permits the locations of the target points to be calculated more accurately. For the thesis experiments, the measurement objective of determining in-plane components related to the FE models leads to an accuracy requirement of $0.001 \mathrm{~cm}$.

\section{Calibrate cameras}

Camera calibration is a crucial process because the accuracy of the measurement depends on how well this task is performed. Camera calibration is defined as the process of identifying intrinsic as well as extrinsic parameters of the camera such as focal length, imaging scale, image center and lens distortion, and calculating their effect on the image projected on to the CCD. Intrinsic parameters are the characteristics of the camera that are inherent to the sensing device and the built in optics. Unlike the intrinsic parameters, the extrinsic parameters depend on the position and orientation of the camera. This is a field calibration process in which the cameras are calibrated in positions, and with distances and test article sizes approximating those of the experiment. The camera parameters describe the geometry and distortion of the projection of a three-dimensional scene on to the two-dimensional CCD of the camera as illustrated in Figure 
5-2. Therefore, calibrated camera parameters are needed by the algorithm to calculate the $3 \mathrm{D}$ points in space from the image points.

For larger test articles (i.e., on the order of 1-10 meters), the camera calibration process starts by taking photographs of a projection on a flat wall of a calibration grid provided with PhotoModeler Pro v4.0. The orientations and locations of the camera or cameras need not be recorded because the software calculates them as part of the calibration process. Photographs of the projected grid are taken and imported into the calibration software. Four uniquely marked points are selected in all the photographs. The projected distance between two of them is given as input to the calibration process. The photographs are then analyzed, which results in the camera intrinsic and extrinsic parameters. For the smaller test articles used herein, photographs of the actual slide were used in the calibration process instead of a projection of the calibration slide. Four of the ten images used for calibration are shown in Figure 5-3.

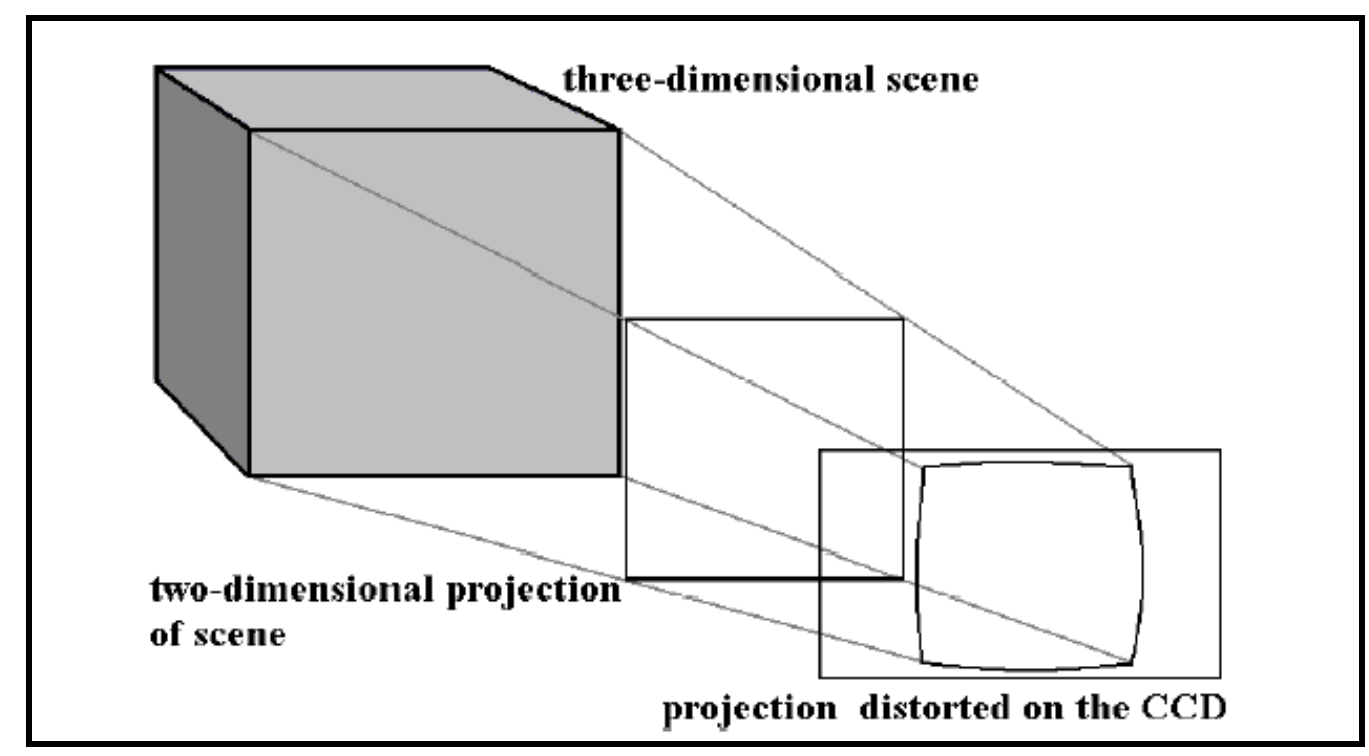

Figure 5-2: The projection and distortion of the real world 3D scene on to the image sensor [82] 


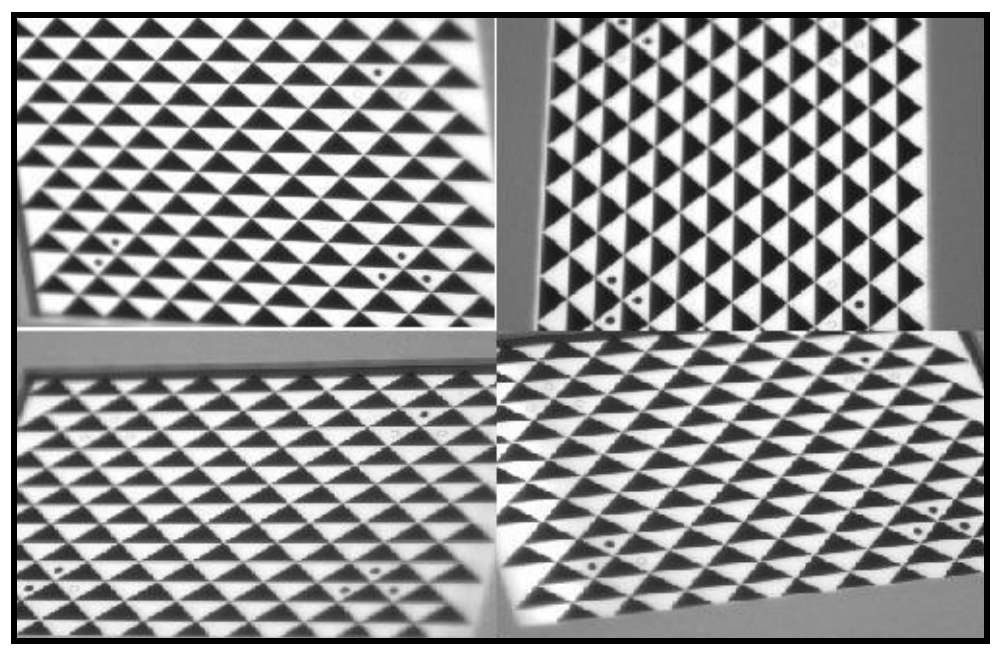

Figure 5-3: Four of the ten images of the calibration slide used for field calibration

\section{Photograph the object scene}

The position and orientation of the cameras when photographing the object scene must consider both the targets on the test article and the accuracy needed. All features to be identified must be included in at least two photographs. The angle between two camera positions is an important consideration in taking photographs of the scene. An angle closer to $90^{\circ}$ minimizes subsequent error. Figure 5-4 illustrates the accuracy differences with different angles between two photographic locations.

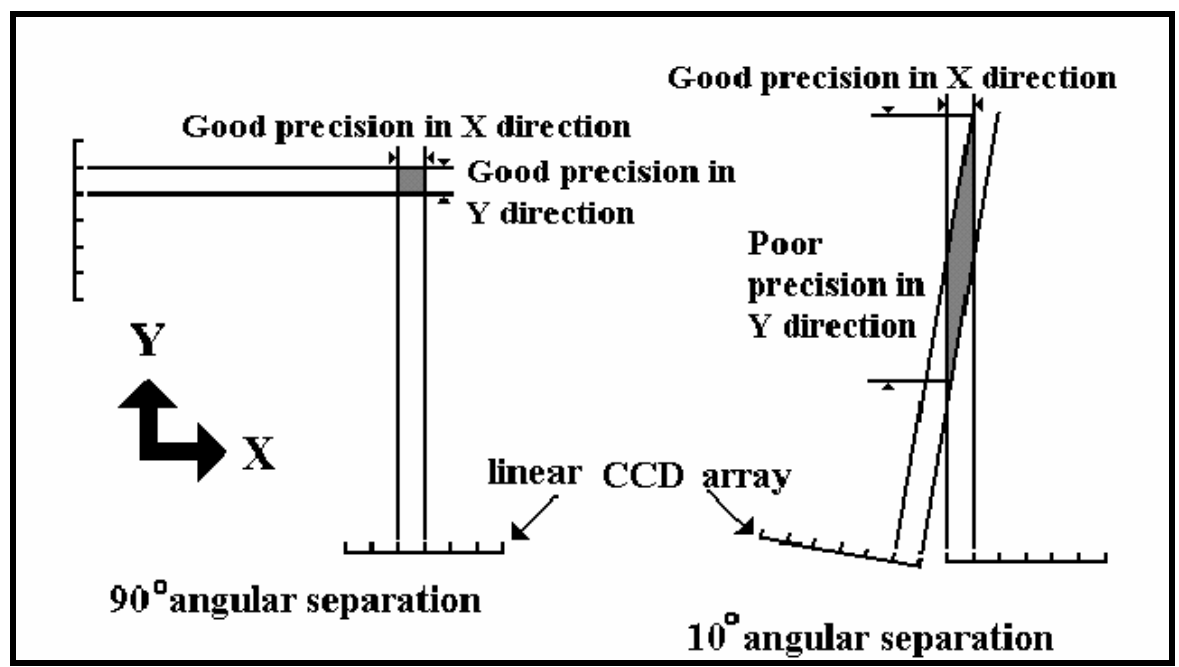

Figure 5-4: 3D location of a point using Triangulation using a linear CCD array with two different angles of separation [82]. 


\section{Transfer photographs from the cameras to the computer}

After photographs of the object scene are taken, they must be transferred to the software. PhotoModeler Pro v4.0 software supports many different formats for images, which is a useful option because different cameras produce images in different formats. Photographs are transferred to the computer's hard drive then read by PhotoModeler Pro V.4.0. After transferring, the photographs are associated with the corresponding camera calibration file(s) to perform further analysis.

\section{Mark the features or the targets on the photos}

Once the photographs have been imported into the software, marking the desired targets is the next important step to be performed. This can be done either manually or, for certain target geometries, using an automated target identification algorithm. The problem associated with manual target marking is that even with high contrast and maximum zoom, a human operator can accurately mark a target to within only one to three pixels.

Automatic marking of circular targets is provided by PhotoModeler Pro V. 4.0. Using the automated centroid target marking system, the accuracy of the results can be improved [82]. Two methods are available for either for dark targets on a light background or light targets on a dark background: 1) centroid method or 2) least squares matching (LSM).

In the current version of PhotoModeler, the centroid method supports any blob shape, but the LSM method works only for circles. Other researchers using projected targets have found that although circles appear as ellipses when projected onto planes that are not perpendicular to the projection, the LSM method calculates the center of the ellipse. Note that this calculated centroid does not correspond to the center of the circular target. For best results, the contrast between the targets and the background must be maximized. Figure 5-5 shows both auto- and manual-selected targets for a test article modified to include both the laser-fabricated pattern and hand-drawn circles. 


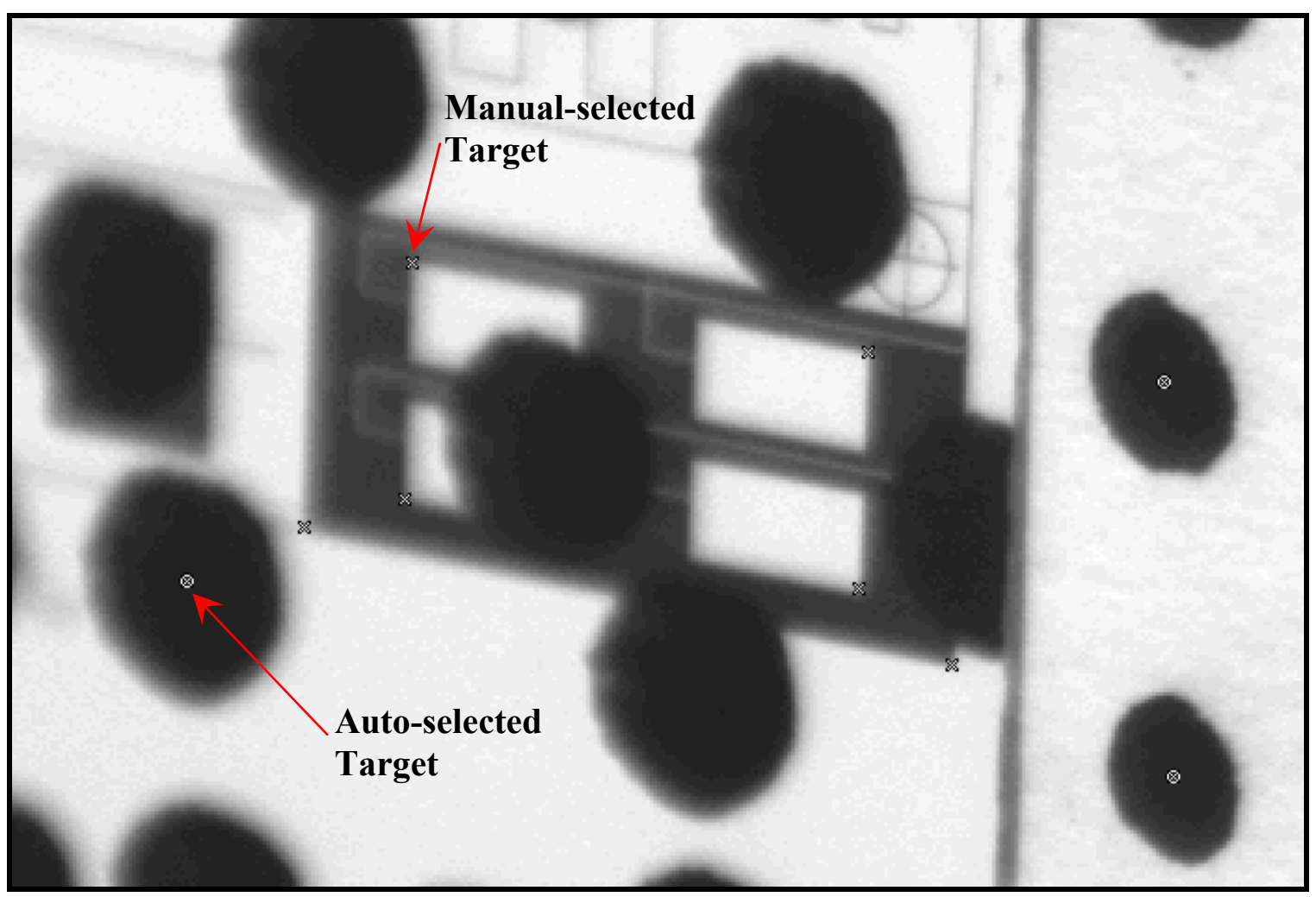

Figure 5-5: Illustration of auto- and manual-selected targets.

Target size is another factor in the marking process. For best results, the target size should be neither too small nor too big. The smallest target size that the PhotoModeler Pro v4.0. can handle is 5 pixels. For better accuracy, the recommended target size should be eight or more pixels. PhotoModeler provides a means to calculate minimum target size [81]:

$$
D_{\min }=\left(8 \text { pixels } * d_{\max } * \text { Format Size }\right) /(\text { Image Size * Focal Length })
$$

where

$\mathrm{D}_{\min }=$ minimum target diameter

$\mathrm{d}_{\max }=$ farthest a target will be from the camera

Format size $=$ horizontal size of the image format

Image size $=$ number of pixels in the image

Focal length $=$ Focal length of the camera used to take images.

The accuracy of the marker depends on the contrast. The accuracy is higher for higher-contrast images and lower for lower-contrast images. Usually LSM is more accurate than the centroid 
method. Figure 5-6 shows the intensity variation typical of a light circular target on a black background.

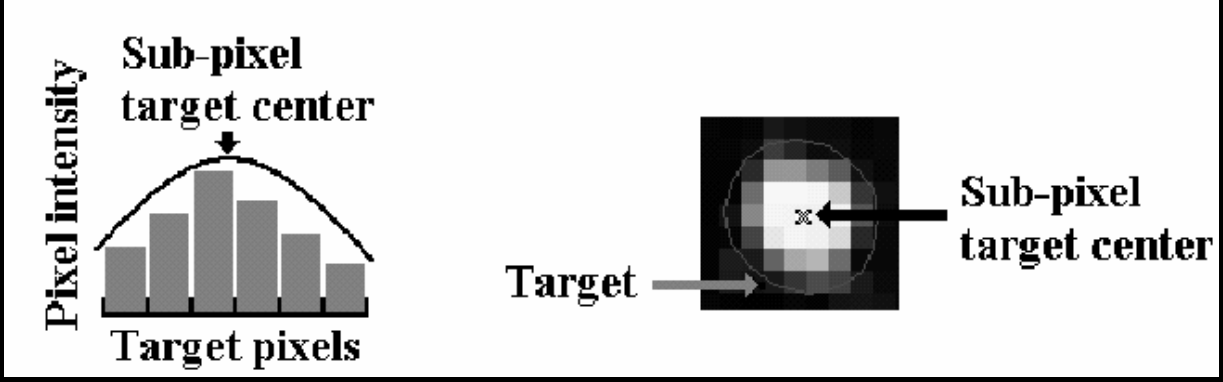

Figure 5-6: The sub-pixel target marker. Note that the center of the calculated peak does not necessarily coincide with the center of a pixel. [82]

\section{Reference points in each image for the same physical point}

Referencing refers to the identification of targets on several photographs that each represent the same physical points on the structure. Referencing consists of identifying corresponding points on two photographs, referred to respectively as a source photograph and a destination photograph. Depending on the number of the targets marked in the image, either manual or auto referencing can be done. Manual referencing of at least ten points is required before the PhotoModeler Pro v4.0 is able to automatically reference the remaining points.

Once the photographs have been marked with manually-referenced points, they are termed oriented. When an oriented photograph is used as a source, then referencing is accomplished along a line technically termed as "epi-polar", a line that appears in the destination photograph if the source point 3D location is not known. If the source point 3D location is known, the cursor automatically goes to the target in the destination photograph to facilitate referencing all target points. It is not necessary that all points appear in all the images, but if 3D coordinates are required, the point must appear in at least two images.

\section{Process the data}

In order to obtain accurate $3 \mathrm{D}$ coordinates of the desired targets, the referenced images have to be processed. Processing not only calculates the 3D coordinates of the points but also calculates the orientations and locations of the camera and adjusts the camera parameters to obtain results 
that are more consistent. This is accomplished in a nonlinear least squares solution with $2 * \mathrm{~N} *$ $\mathrm{n}$ equations in $(6 * \mathrm{~N})+(3 * \mathrm{n})+(8 * \mathrm{c})$ unknowns, where $\mathrm{N}$ is the total number of camera locations, $\mathrm{n}$ is the number of points and $\mathrm{c}$ is the number of cameras being calibrated. [82]

\section{Export results}

In PhotoModeler Pro v4.0, the processed data can be exported in several formats. The 3D data, 2D data from a projection of the $3 \mathrm{D}$ data on to a plane, error files that quantify measurement accuracy, and camera information can be exported for further analysis.

\subsection{Photogrammetry of Patterned Thin-Film Mirrors}

Evaluating photogrammetry for feasibility to measure dynamic response of thin-film mirrors involved taking pictures of the patterned thin-film mirrors from different locations and orientations. In all, 15 test articles were fabricated and 12 were patterned for testing. Two different experiments were developed: 1) a static deflection experiment with an applied force at the center of the patterned thin-film mirror and 2) a precision in-plane translation experiment. Photographs were taken for each experiment from four different locations or orientations. Figure 5-7 is one sequence of four photographs taken for photogrammetry analysis. This sequence is for the eighth of ten load steps.

Figure 5-8 shows a photograph of the patterned thin-film mirror marked with the lines that were used to quantify the in-plane displacement and for comparison with FE predictions. Fourteen different lines were identified in each photograph; twelve are seen in Figure 5-8. 

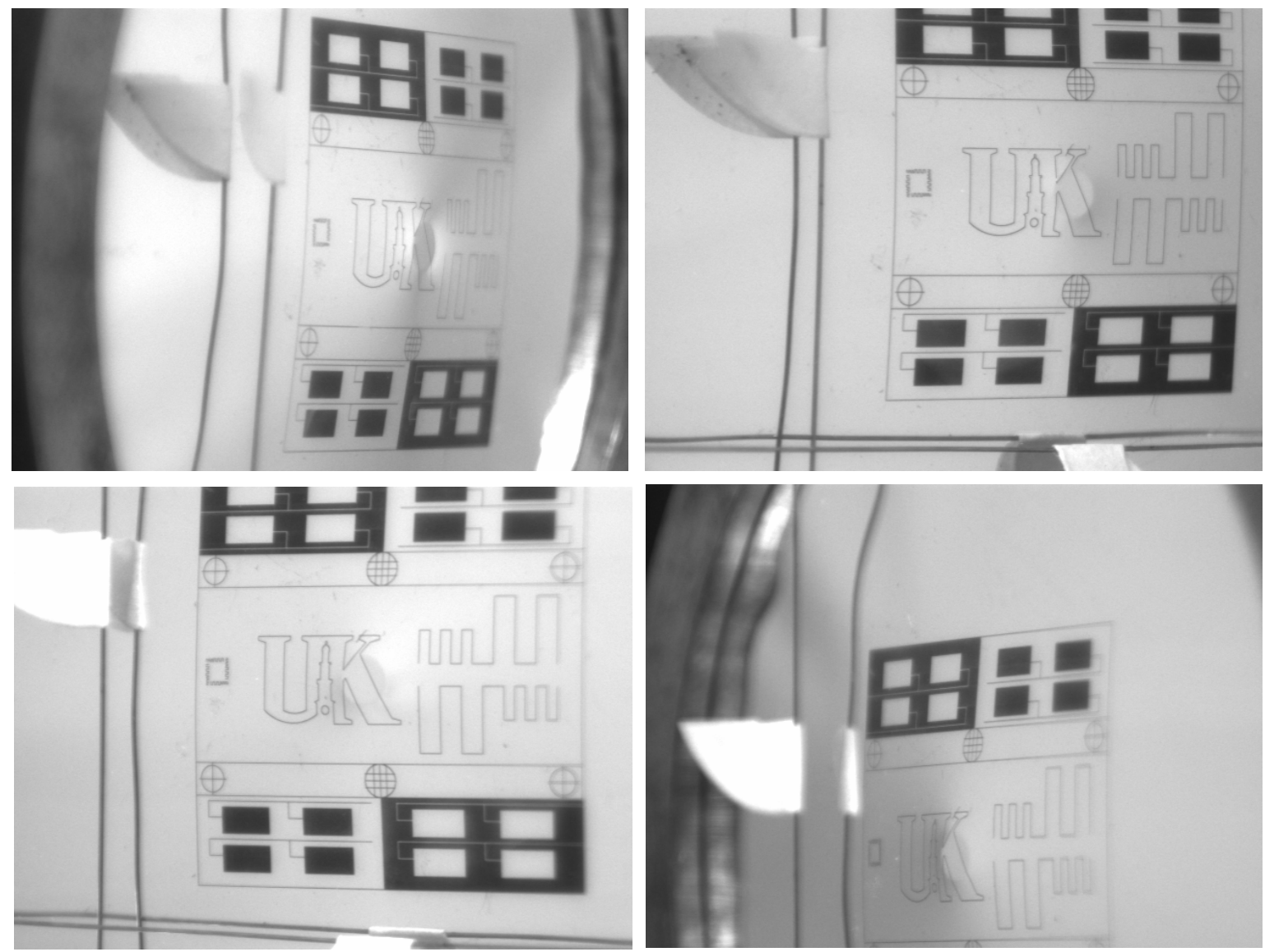

Figure 5-7: Photographs taken for photogrammetry analysis at force level of $8 \mathrm{~N}$ 


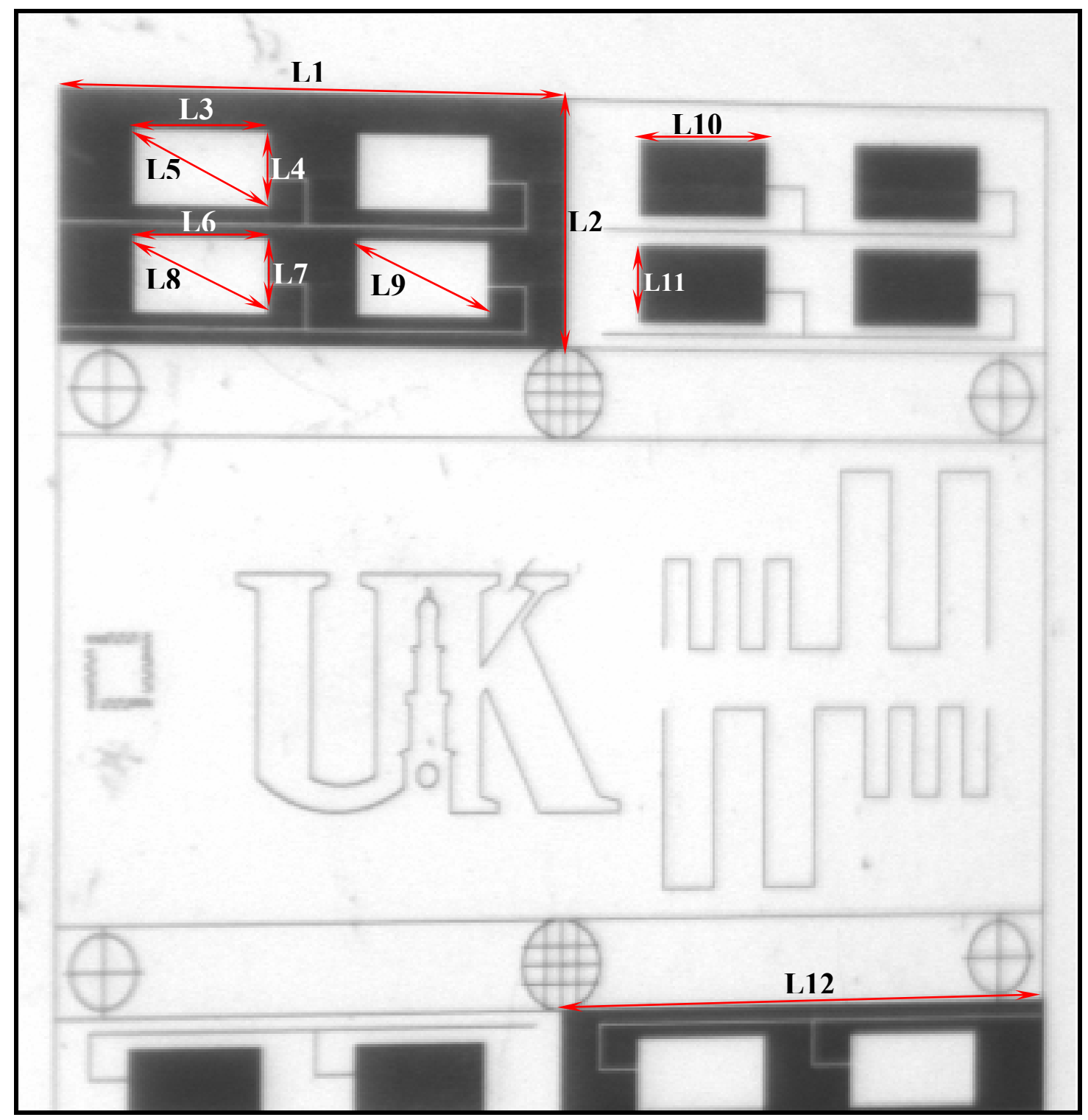

Figure 5-8: Photograph marked with lines that were used to quantify the in-plane displacement of thin-film mirrors.

Figure 5-9 and Figure 5-10 each show the front and side views of 3D reconstructions of the patterns on the thin-film mirror for the no-load case and maximum-load case, respectively. All fourteen lines, along with several other points that were reconstructed, can be seen in the figures. The side view in Figure 5-9 shows no displacement of the pattern since all the points lie in a plane under no load. In Figure 5-10 the side view shows the out-of-plane displacement of the points and lines. The reference points can also be seen in the photographs. Though the change in 
length of only fourteen lines was of interest, several other points were used to increase the efficiency and accuracy of measurement.

Figure 5-11 and Figure 5-12 show the camera orientations and locations for the two experiments performed to evaluate feasibility of the photogrammetric system. Figure 5-11 shows the camera locations in a plane parallel to the ground for the first experiment. For the second experiment, the four camera locations included two vertical heights are seen in Figure 5-12. Although Figure 5-11and Figure 5-12 illustrate the camera locations, the PhotoModeler Pro v4.0.locates the cameras as part of the data processing step.

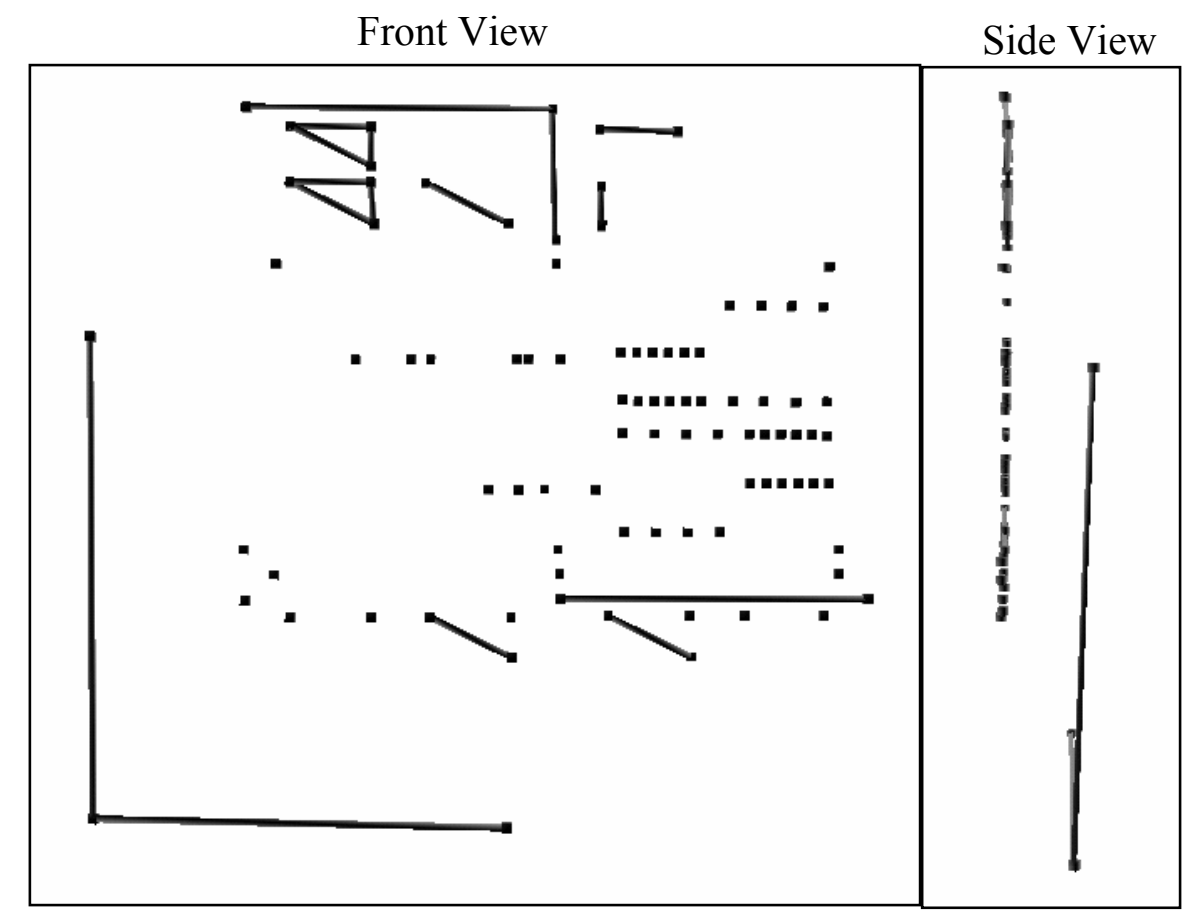

Figure 5-9: 3D reconstruction of the thin-film mirror using photogrammetry when no force was applied. Front view (left); Side view (right) 
Front View

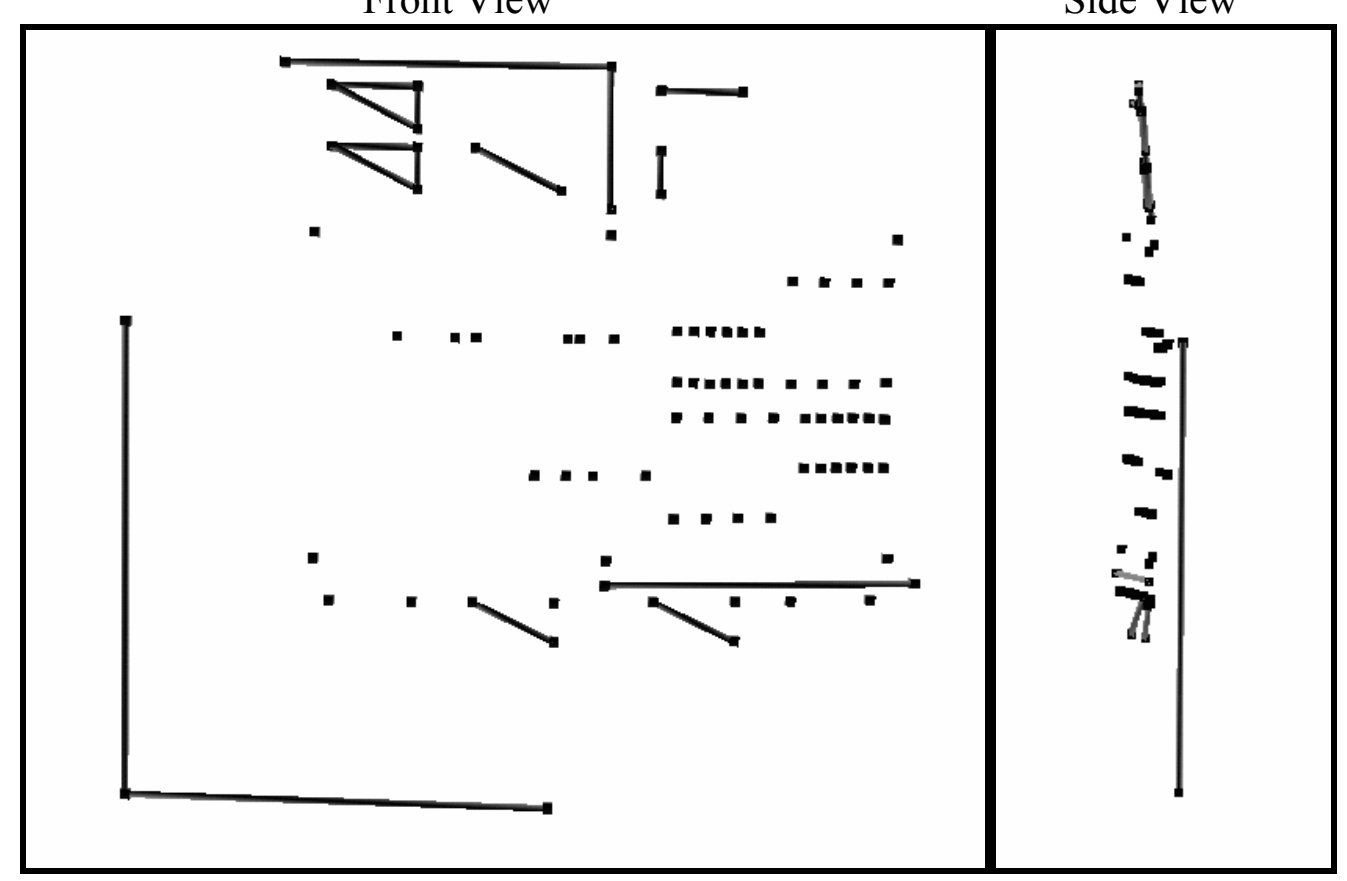

Figure 5-10: 3D reconstruction of the thin-film mirror using photogrammetry when force of $10 \mathrm{~N}$ was applied. Front View (left); Side view (right)

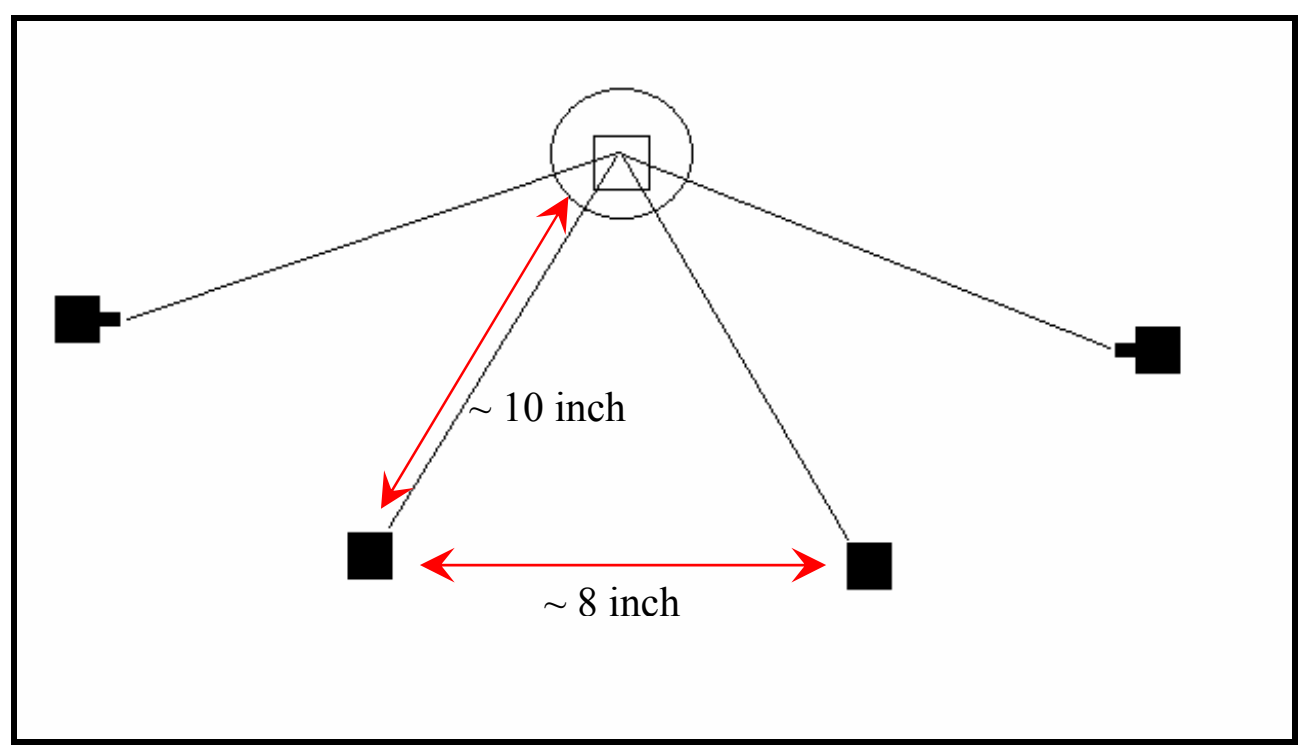

Figure 5-11: Camera locations for the experiments with the force sensor 


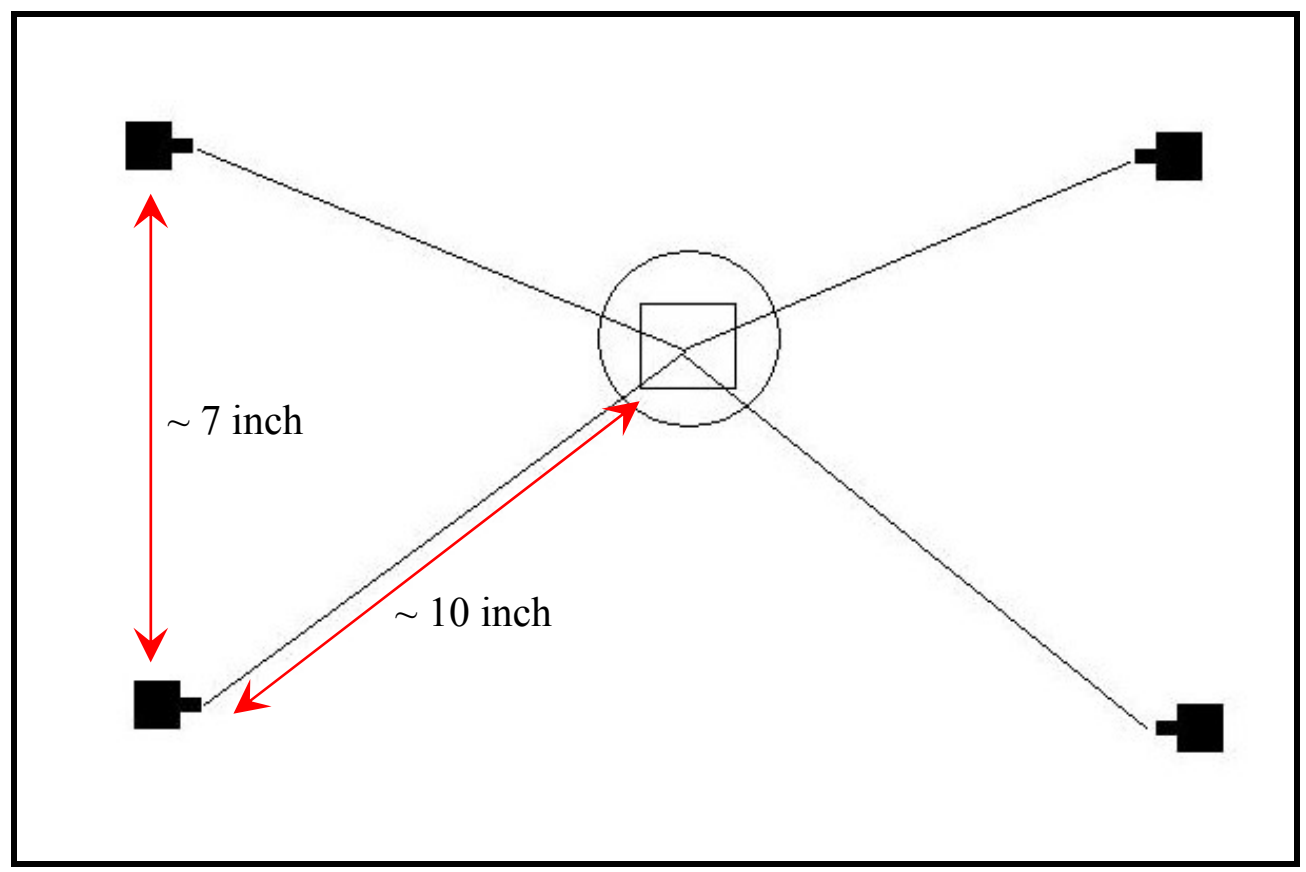

Figure 5-12: Camera locations for the translation experiments

Copyright (C) Phanikrishna Thota 2003 


\section{Chapter 6：STATIC TESTS}

\subsection{Introduction}

As a first step towards achieving our goal of dynamic, in-plane displacement measurement of patterned thin-film mirrors, static displacement experiments were done using photogrammetry. Dynamic in-plane displacement tracking will be performed by performing photogrammetry on synchronized image sequences taken from multiple camera positions. In order to provide a strong baseline for the dynamic tests, preliminary static tests were conducted on patterned thin-film mirrors. Two different experiments were performed. In the first tests, thinfilm mirrors were subjected to a point load at the center that deformed the thin-film test articles both in-plane and out-of-plane. The force was applied as a distributed load over a small circular region of diameter 0.138 inch and measured using a calibrated load cell interfaced with a computer data acquisition system. In the second tests, the thin-film test article was precisely translated in-plane. Three translation experiments were performed to study the effects of camera location and orientation and sub-pixel target marking on the accuracy of measurement.

\subsection{Camera Description}

A SONY XC - 55BB (Figure 6-1) camera was used to take pictures of the statically deformed or translated test articles. The remote head of the monochrome camera has a precisely installed CCD that ensures accurate alignment. The camera has a $1 / 3$ " interline transfer progressive scan CCD. The CCD uses square pixels, which provides equal resolution in both the horizontal and vertical axes, and eliminates the necessity of dimensional correction for image processing. A $16 \mathrm{~mm}$ lens with an extender (Computar EX2C) that doubles the focal length when attached to the rear of the lens was used to obtain better images. This made the value of the focal length $32 \mathrm{~mm}$. The camera has a resolution of 659 pixels in the horizontal direction and 494 in the vertical direction. The size of each pixel (cell size) is $7.4 \mu \mathrm{m} \times 7.4 \mu \mathrm{m}$. The camera was interfaced to a computer so that the images taken were initially saved as image files and then converted to the TIFF format for subsequent analysis. 


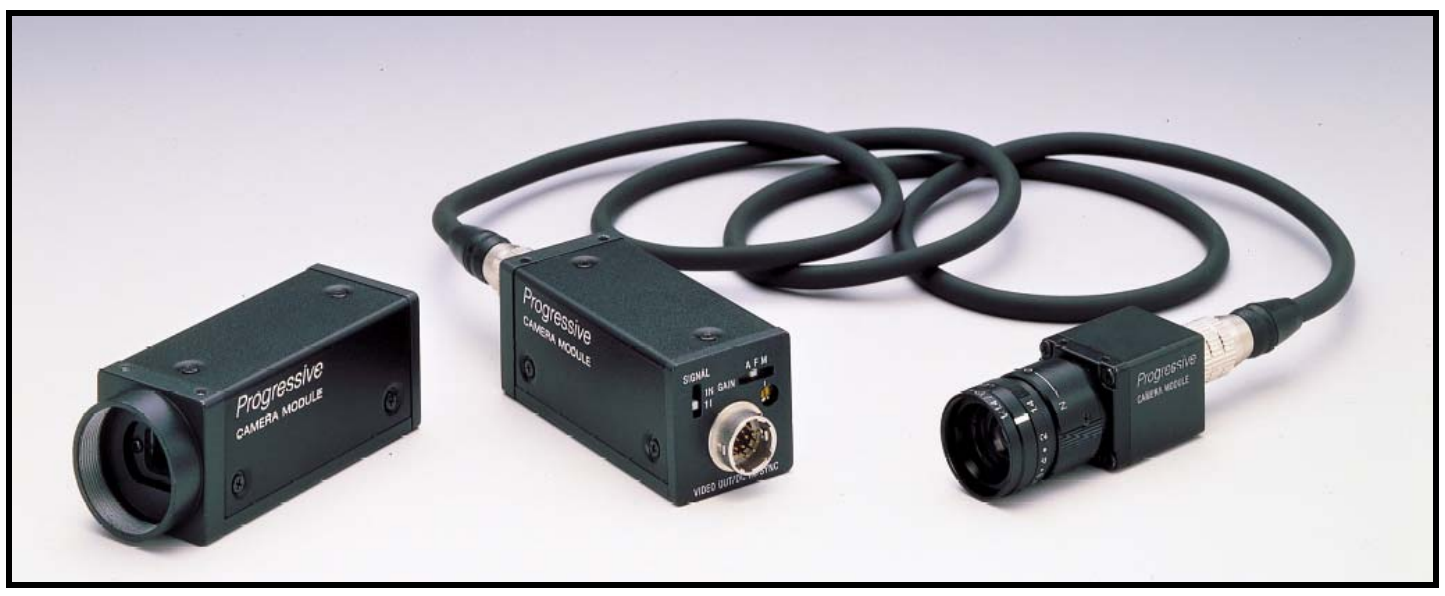

Figure 6-1: Sony XC-55 BB camera used to take photographs of the statically displaced thin-film mirror [83].

\subsection{Deformation Experiments}

Figure 6-2 shows the experimental setup used for the first static experiments. The thinfilm mirror was clamped in a variable lens holder, which was designed to hold optics gently but firmly within a kinematically designed V-shaped mounting base. The grip force was adjusted by changing the height of the crossbar, which featured a long alignment bore and guide adjustment that prevented jamming or wobbling during height adjustments. The load cell was coupled to a high-performance, low-profile, ball-bearing linear stage. The micrometer on the stage permitted accurate and controlled motion of the load cell. The entire setup was built on an optical breadboard to permit relocation of the apparatus. Two sets of images and data were recorded, one with reference points included and the other without them in the scene.

Camera calibration was required in order to enable precise measurement of the surface deflections to be calculated using the photographs. One step in this process is to know the format size of the photographs. That requires an image of a blank sheet of paper. In typical close-range photogrammetry applications, a standard $8.5 \times 11$ inch sheet of paper is used to provide the format size. For this ultra close-range photogrammetry effort, a sheet of paper that was $0.75 \mathrm{x}$ 1.00 inch was used instead as seen in Figure 6-3. For comparison, it is shown adjacent to a standard 12" ruler. The second step involves a calibration image. Here, the size of the test article and the size of the features on the PhotoModeler Pro v4.0 calibration slide were comparable in scale; as a result, the slide itself (rather than its projected image) was used for calibration. The 
camera calibration results were imported in to the PhotoModeler Pro v4.0 software. The accuracy of the resulting 3D model reflects the accuracy of the calibration procedure.

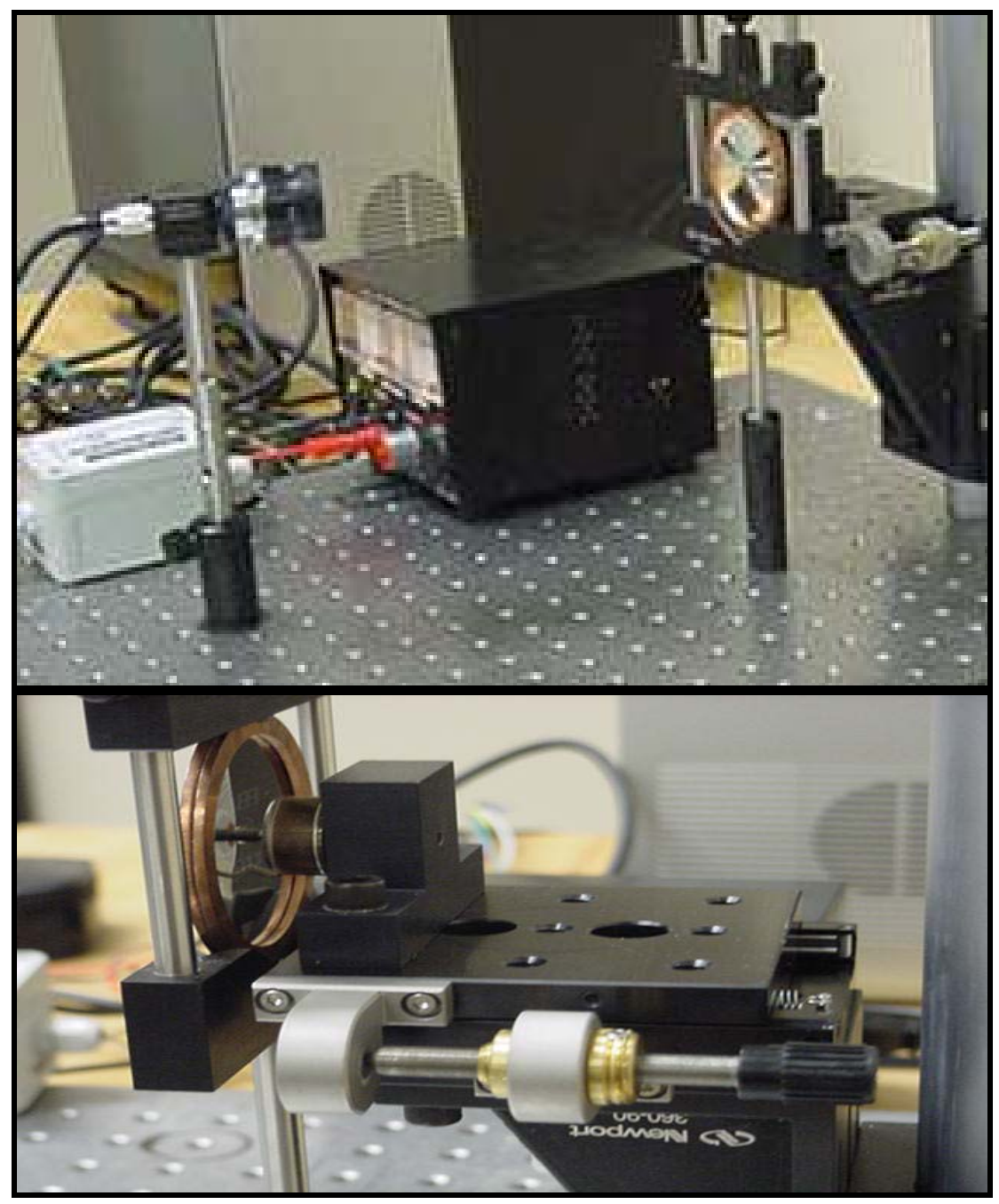

Figure 6-2: Experimental set-up for the in-plane displacement measurement of thin-film mirrors showing the camera and the test article (top), and detail of the load cell (mounted on a linear stage) forcing the test article (bottom). 

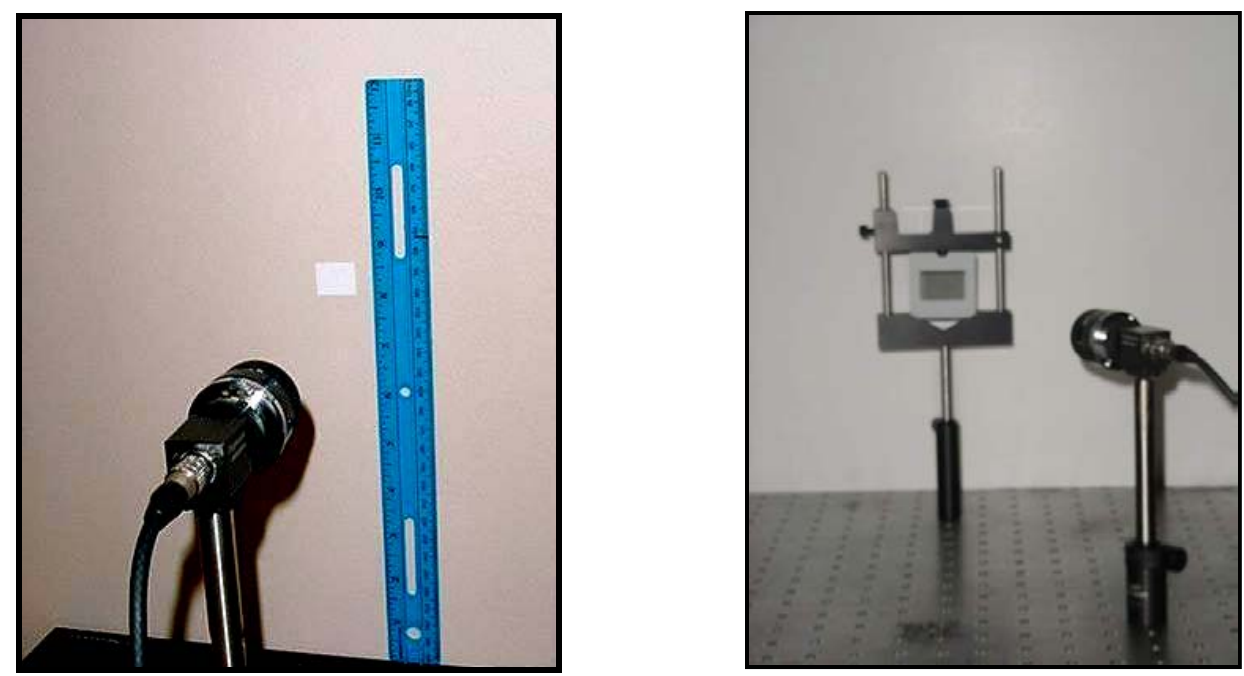

Figure 6-3: SONY XC - 55BB camera, standard 12 inch ruler, 0.75" X 1.00" paper (left), and calibration slide (right) imaged during the calibration process.

One problem encountered while taking pictures was that the surface texture of the thinfilm mirror changed with camera position. This occurred because the surface texture of the mirror was essentially comprised of a reflected image, and caused some of the features in the pattern to vanish in a number of the photographs. This problem was resolved by positioning a white board opposite to the camera position, so that the image reflected by the film consisted primarily of a solid white background. In order to maximize contrast in the images and to obtain clearly defined targets, a black sheet was placed at the back of the mirror. This was done so that the areas where the mirror was transparent because of the feature fabrication would appear black in the image and the rest would appear white.

Ten sets of four photographs were taken of each of the two test articles, with each set corresponding to a different applied load. Each load was expected to produce a different level of deflection on the surface of the test article. All of the photographs were taken using the same camera settings. No photographs were taken on the line normal to the undeformed surface of the test article, since that would have caused a reflection of the camera to appear in those images.

No fixed reference points were provided in the first set of images. The force application area was considered as reference but found to be in adequate. Consequently evaluation of inplane displacement was not possible because every point in the image was moving relative to all 
other points. Hence, another set of images was taken including reference points that remained stationary in all load step images.

Crossed wires were used to provide reference points for this set of images. Figure 6-4 shows the crossed wires in position adjacent to the mirror surface. Crossed wires were chosen for this purpose because the reference had to be within the camera's field of view and its depth of field as seen in Figure 6-5. Hence, the crossed wires were placed as near as possible to the surface of the mirror and to the etched test pattern. Grooves were machined into the copper ring in order to firmly fix the crossed wires into position.

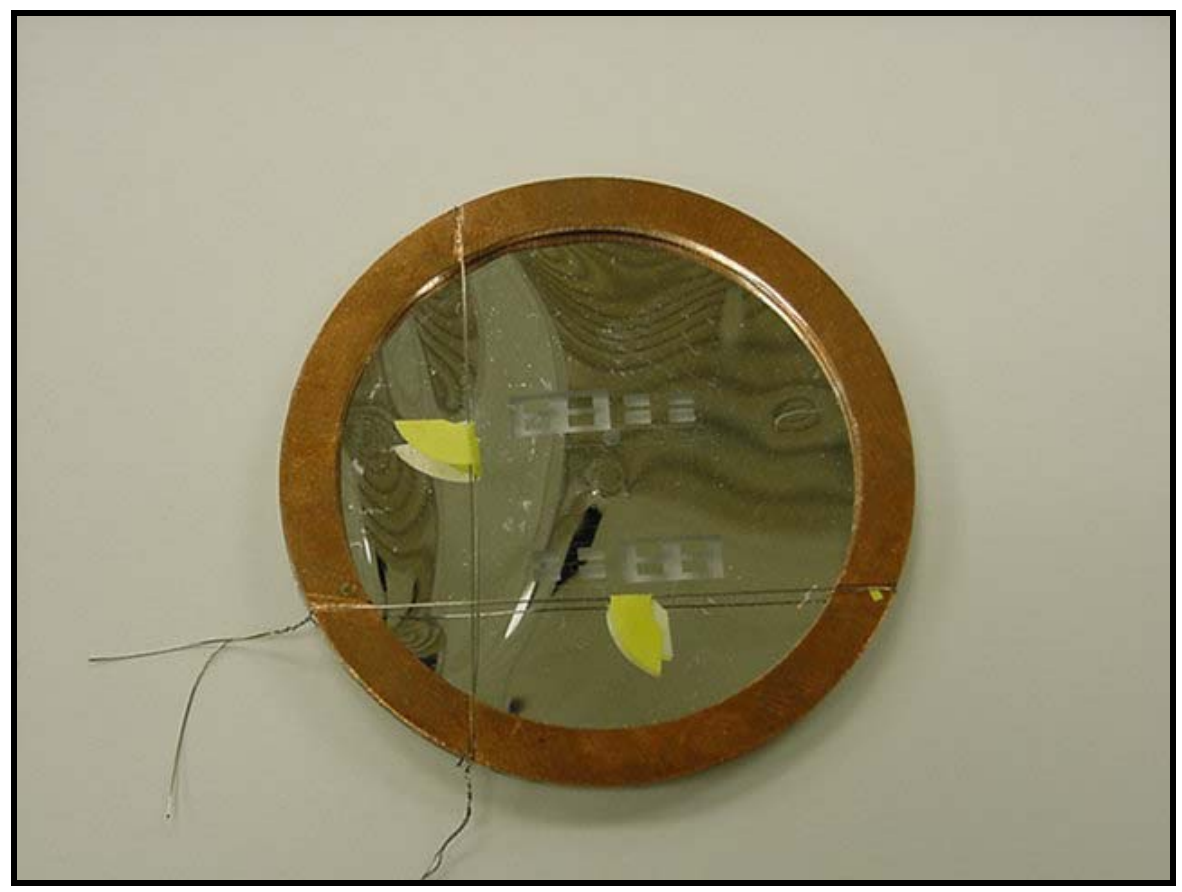

Figure 6-4: Thin-film mirror with the crossed wires used as reference points. 


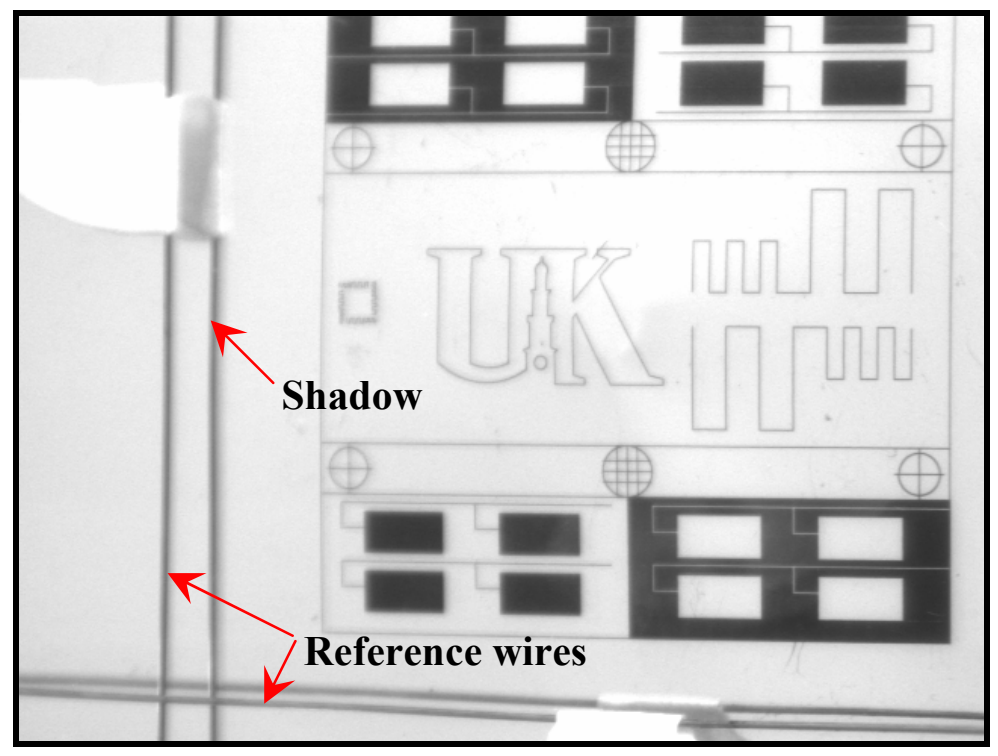

Figure 6-5: One of the photographs from the final data set. Initial images were similar, but without the reference wires.

\subsubsection{Results of the Deformation Experiments}

Figure 6-6 shows the photographs of the forced thin-film mirror from one camera location for four different force levels. Note that when the load was applied to the center of the test article, the indicated force reading gradually decreased during the process of taking photographs because of the yielding of the thin-film mirror. The deformed thin-film test results and ANSYS static analysis results were compared. Plots were drawn of force versus the normalized change in line length for all the fourteen lines defined in Chapter 5. The length of the fourteen lines of interest defined in the pattern in the previous chapter were "measured" using photogrammetry for the $0 \mathrm{~N}$ load case. Table 6-1 summarizes these results. The "fabricated length" is the length of the line on the original CAD drawing of the pattern. Two of the fabricated lengths were checked using an optical microscope and found to be as follows: line 3 $0.253 \mathrm{~cm}$ and line $4-0.124 \mathrm{~cm}$. The line lengths identified by photogrammetry are comparable to the fabricated lengths. Figure 6-7, Figure 6-8, Figure 6-9, and Figure 6-10 present the comparison between experimental and numerical results for lines $2,5,8$, and 14, respectively. These were selected as representative of all results. The experimental values for change in line length were generally larger than the values obtained from ANSYS. Beyond that, the experimental results do not compare well with the analysis. The best result seen is for line 8 with 
the exception of certain points. Note that, normalized change in line length showed an increasing trend for all lines with force in ANSYS results except one line, line 14.

Table 6-1: Fabricated and measured line lengths for the fourteen lines at $0 \mathrm{~N}$ load

\begin{tabular}{|c|c|c|}
\hline Line No & Fabricated Length $(\mathrm{cm})$ & Measured Length (cm) \\
\hline 1 & 0.981 & 0.989 \\
\hline 2 & 0.464 & 0.434 \\
\hline 3 & 0.244 & 0.257 \\
\hline 4 & 0.122 & 0.126 \\
\hline 5 & 0.273 & 0.281 \\
\hline 6 & 0.244 & 0.258 \\
\hline 7 & 0.122 & 0.127 \\
\hline 8 & 0.273 & 0.285 \\
\hline 9 & 0.273 & 0.286 \\
\hline 10 & 0.244 & 0.254 \\
\hline 11 & 0.122 & 0.127 \\
\hline 12 & 0.981 & 0.978 \\
\hline 13 & 0.273 & 0.286 \\
\hline 14 & 0.273 & 0.287 \\
\hline
\end{tabular}



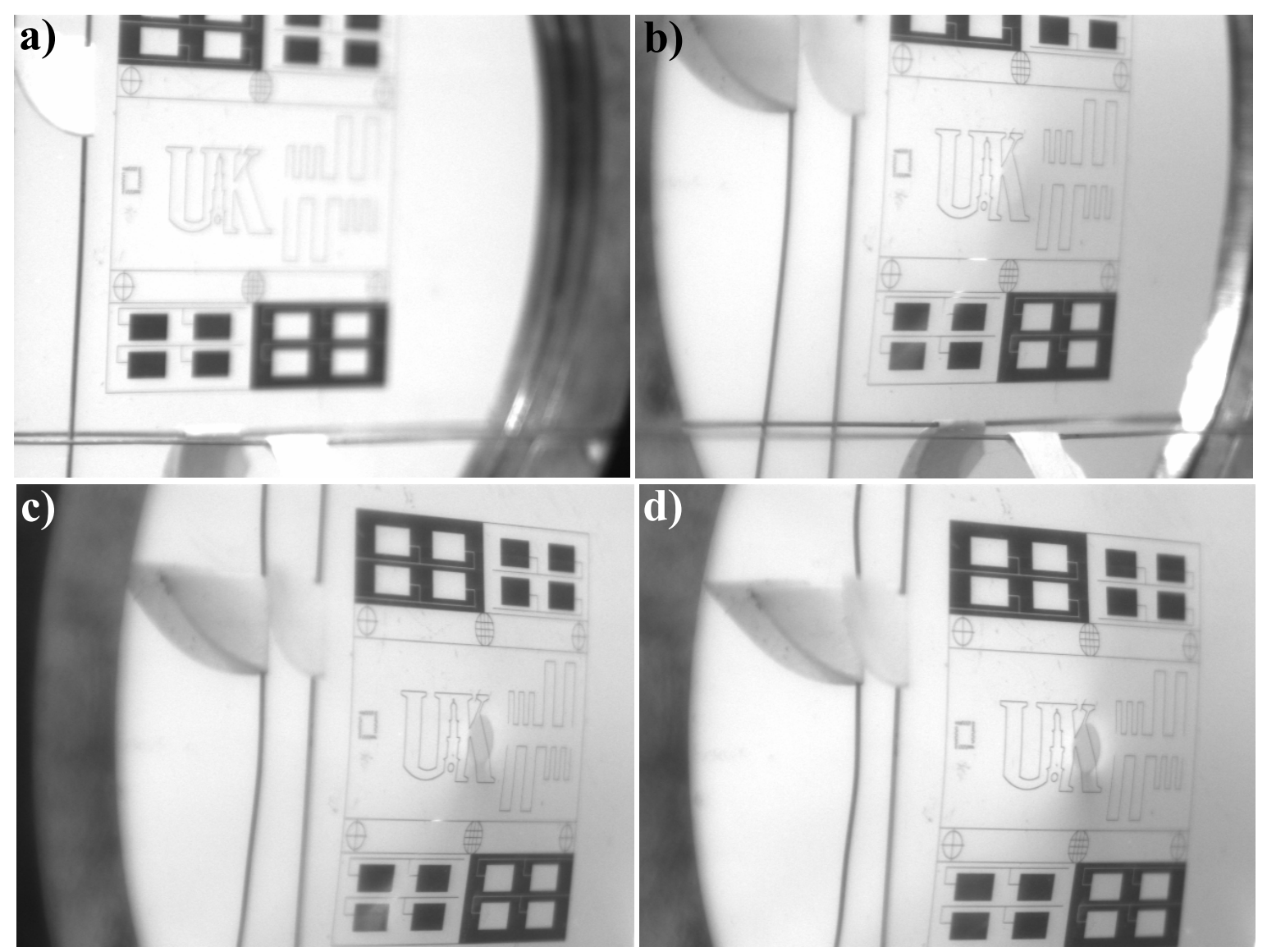

Figure 6-6: Photographs from one camera location for force different force levels: a) $0 \mathrm{~N}$; b) $2 \mathrm{~N}$; c) $6 \mathrm{~N}$; d) $8 \mathrm{~N}$ 


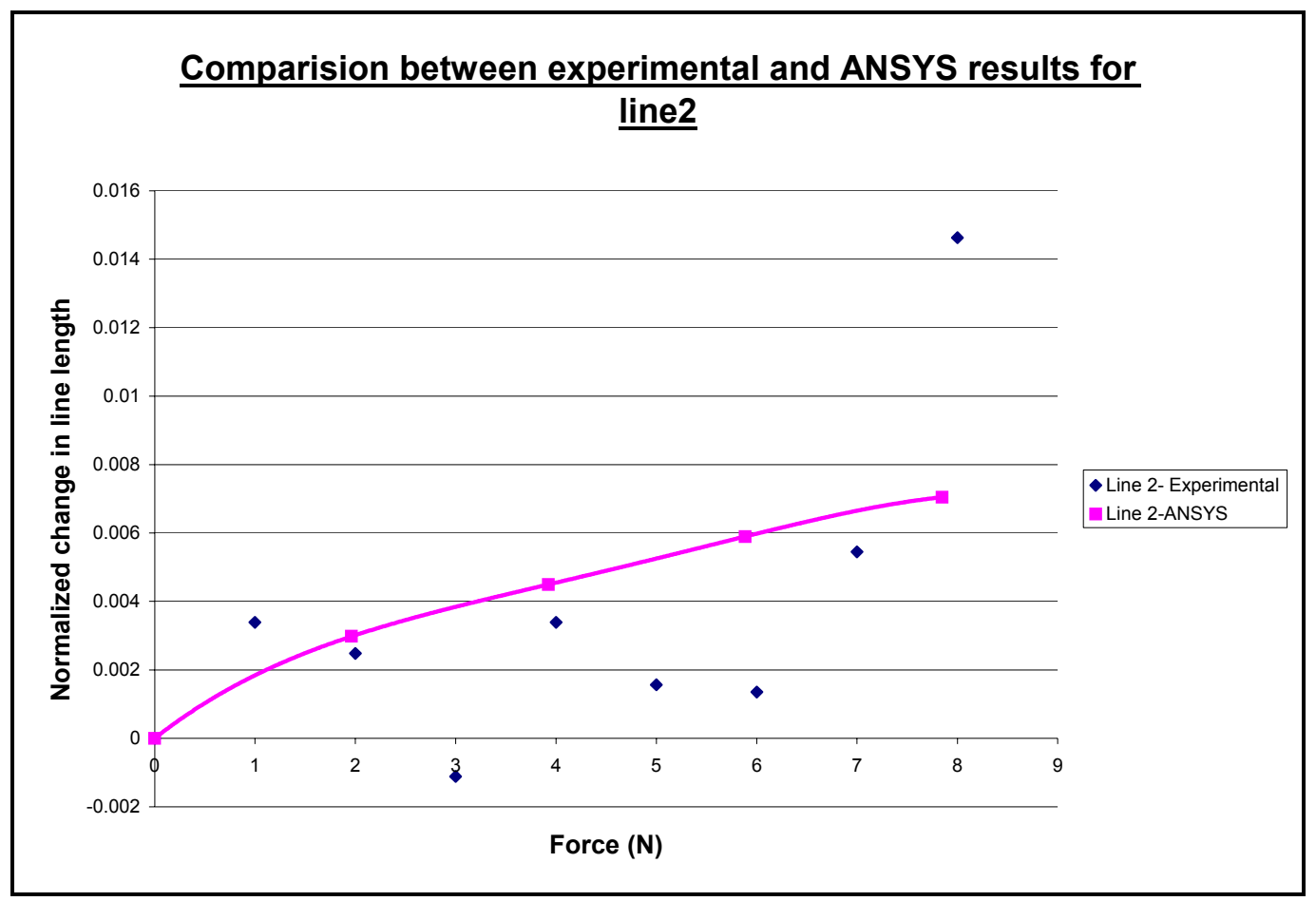

Figure 6-7: Comparison between experimental and ANSYS results for line 2

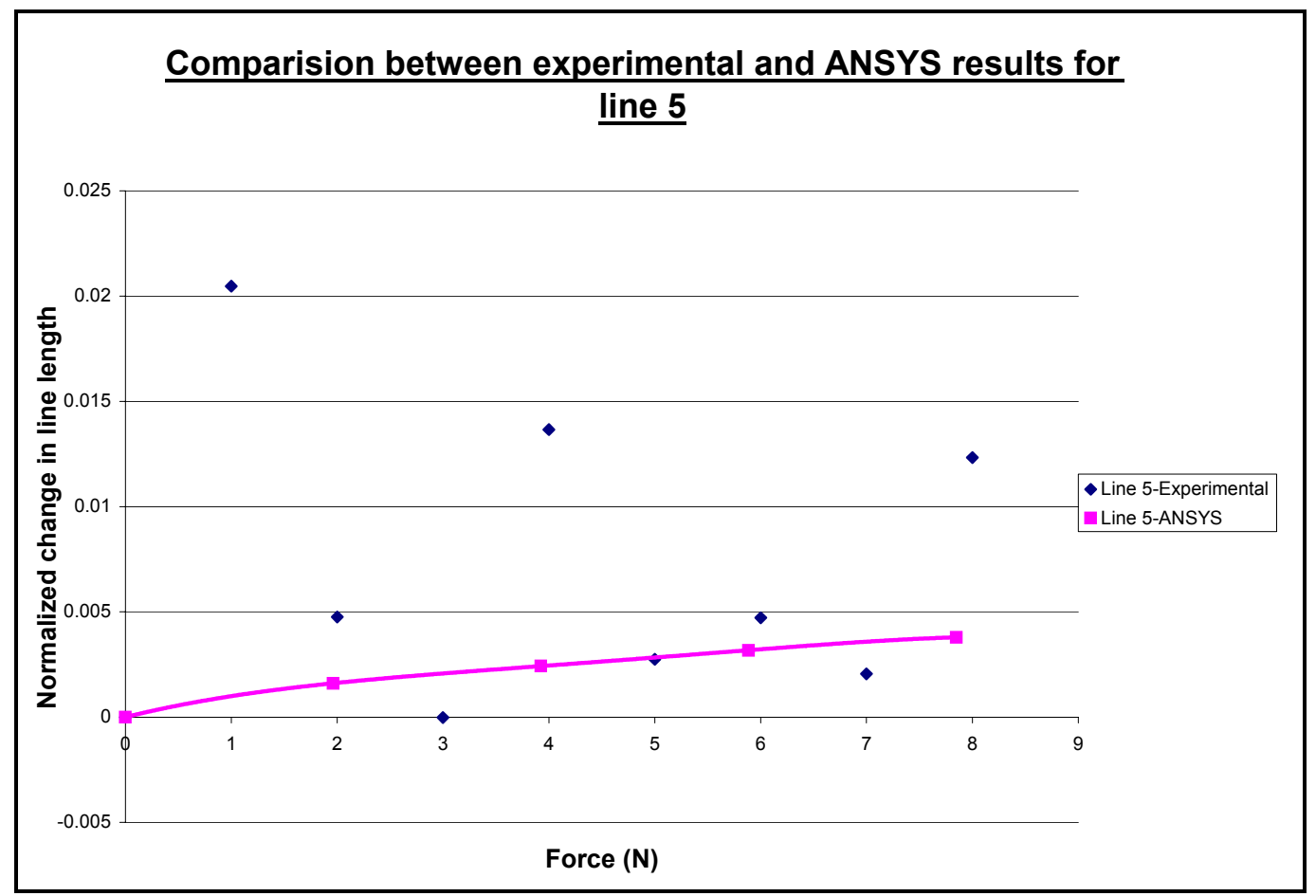

Figure 6-8: Comparison between experimental and ANSYS results for line 5 


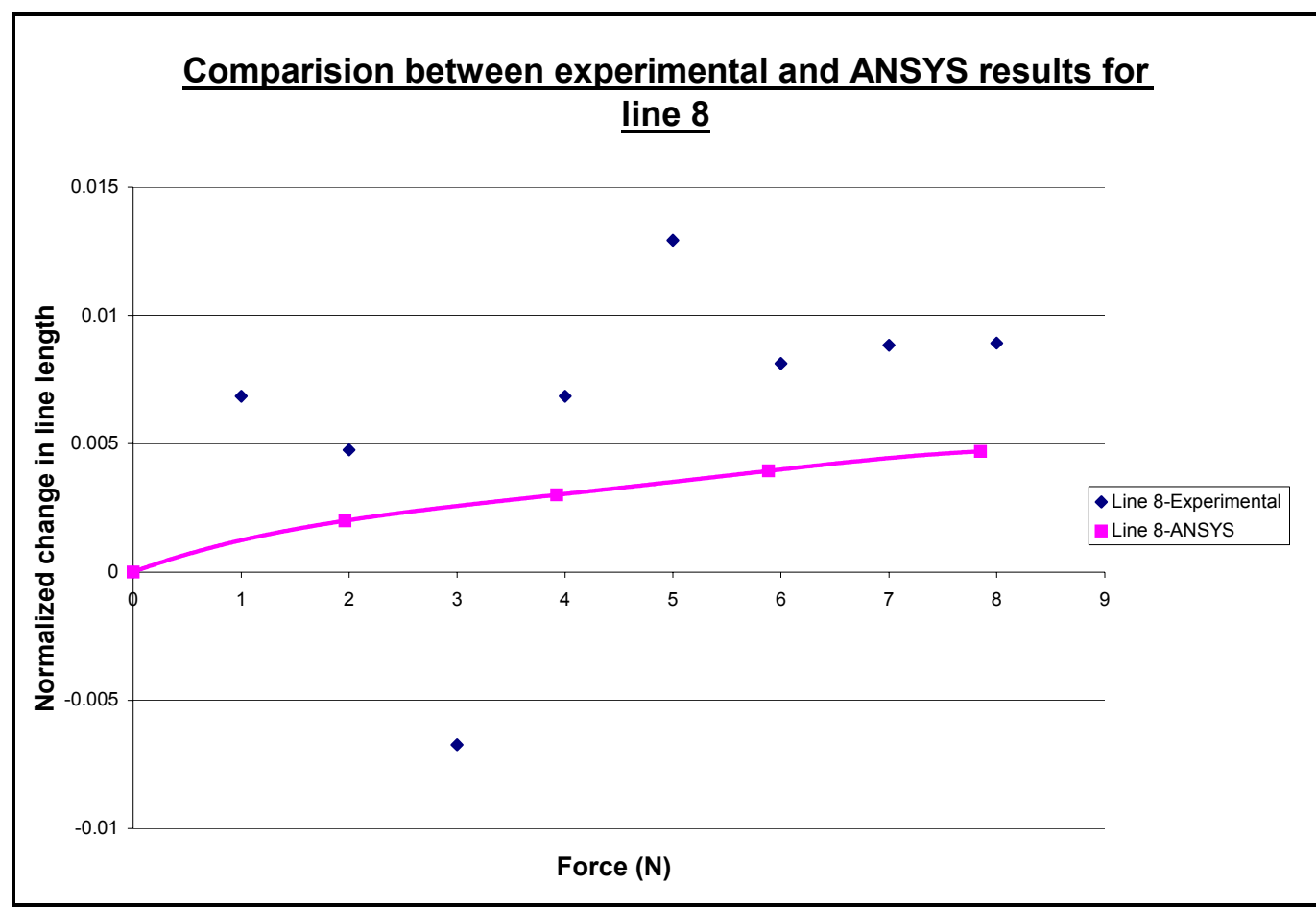

Figure 6-9: Comparison between experimental and ANSYS results for line 8

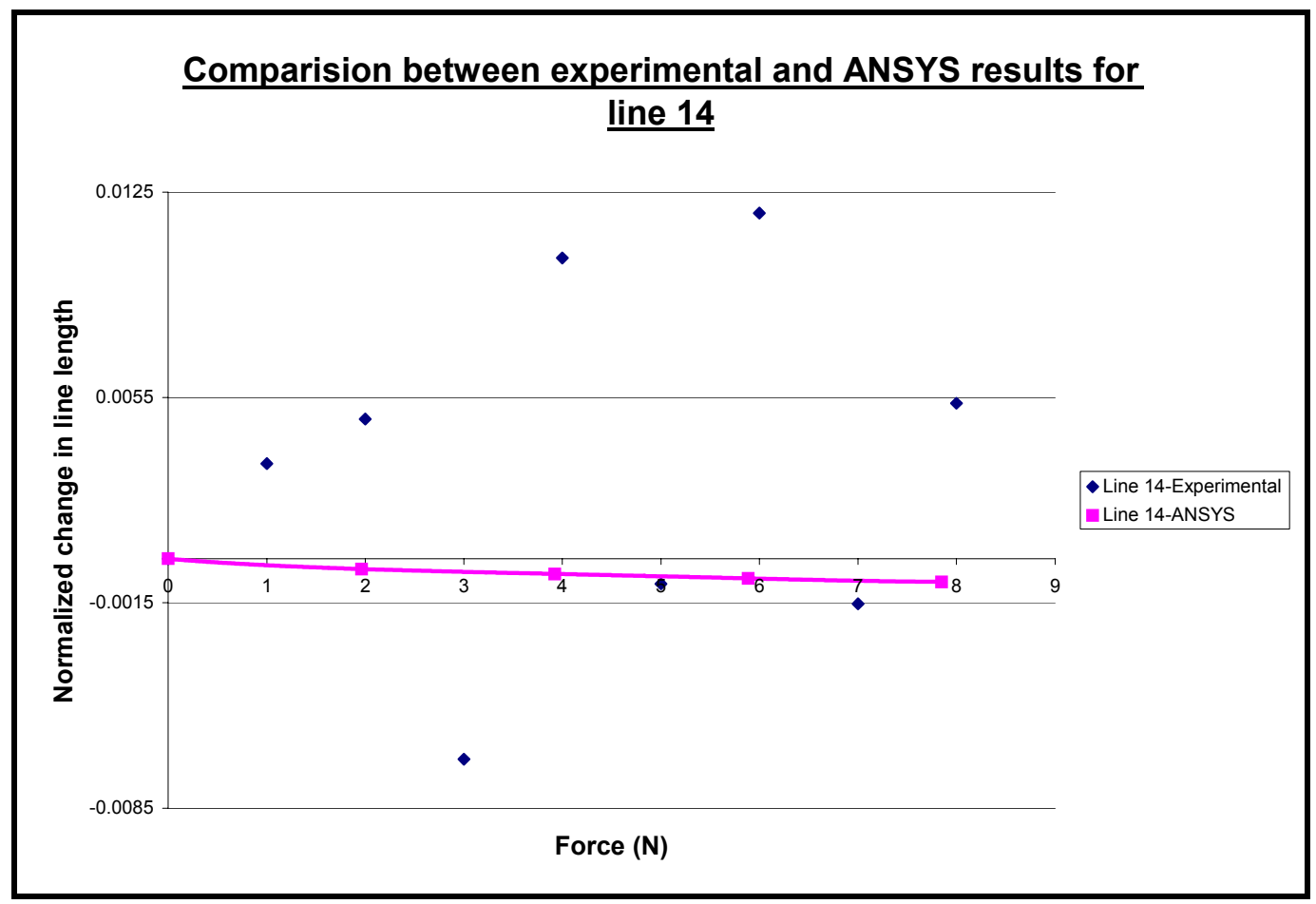

Figure 6-10: Comparison between experimental and ANSYS results for line 14 
Prompted by the discrepancies between the model results and experimental results, two explanations were investigated. First, the maximum Von Mises stress obtained from the finite element analysis was examined. The maximum stress of $718 \mathrm{MPa}$ at $2 \mathrm{~N}$ force level, is greater than the yield strength of the polyester film, which is about $35.2 \mathrm{Mpa}$. This, along with permanent deformation see in the test articles, was a clear indication that the material was forced into its plastic region at the center where maximum stress occurred. This was not modeled.

The second explanation required a closer look at the camera and photogrammetry resolution. The results of Table 6-1 show photogrammetry results comparable to fabricated lengths. Figure 6-11 shows the number of pixels in a known distance on the pattern. This was used to find the ground sample distance (GSD) of the camera. The GSD was calculated from five different distances and averaged to minimize manual vision errors in selecting the number of pixels in the known lengths. The resulting GSD is $0.005125 \mathrm{~cm}$, larger than the maximum predicted displacement for these tests.

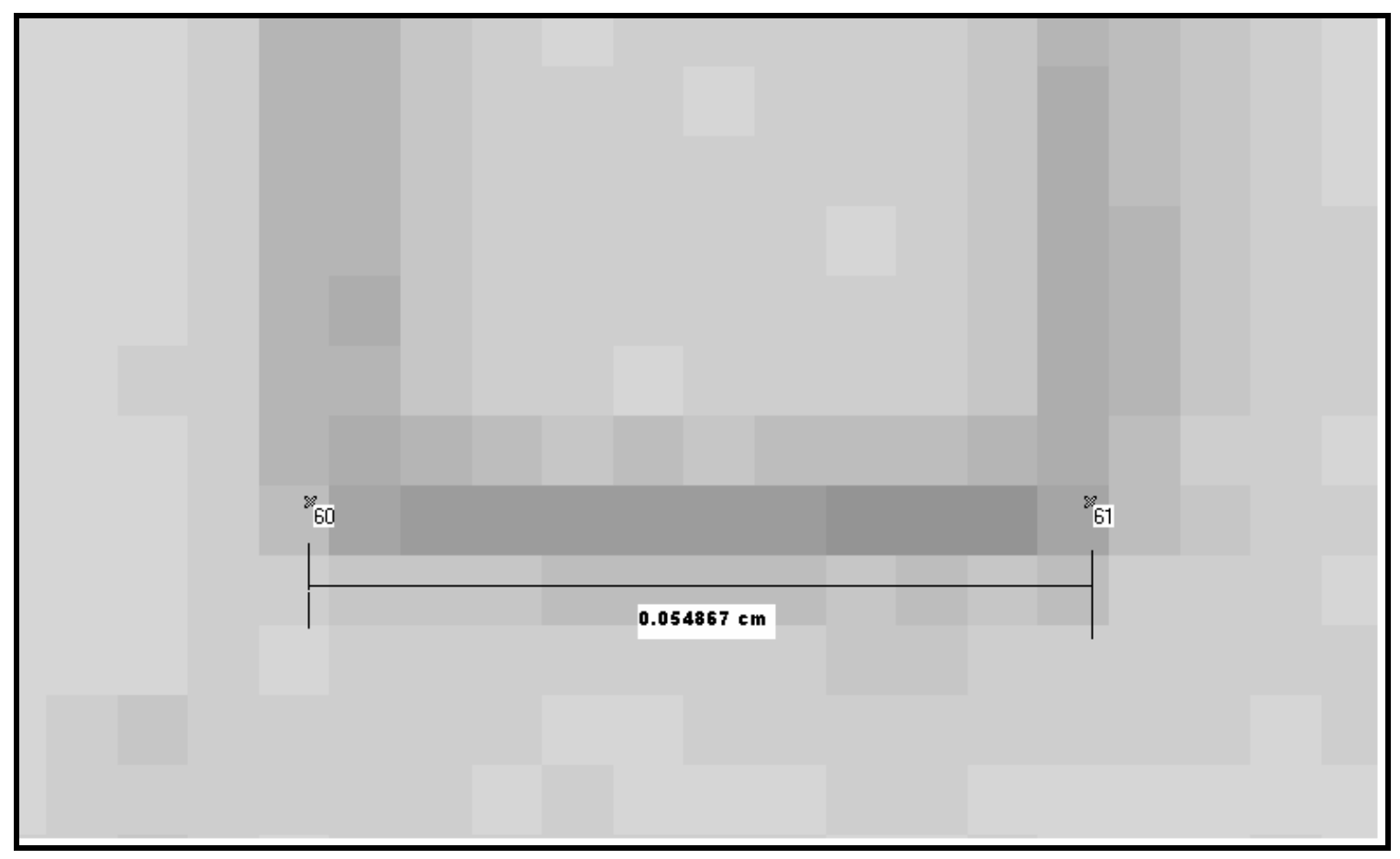

Figure 6-11: Ground sample distance of the camera 


\subsection{Translation Experiments}

To further investigate resolution and accuracy issues, three translation experiments were conducted by applying pure translation to the mirror within its own plane. These experiments were performed to study the effects of camera location and orientation and sub-pixel target marking on the accuracy of measurement. The translatory motion in these experiments was achieved using a linear stage to which a micrometer was attached. Circular areas of different sizes were added using a permanent marker on the mirror as targets along with the fabricated pattern. Additional "dots" were attached by gluing a piece of paper with smaller-size dots printed on it.

For the first of the three translation experiments, movement was applied to the linear stage in steps of $1 \mathrm{~mm}$. Photographs of the mirror were taken from four different locations in two planes to reproduce the 3D model of the mirror surface at each step. The camera locations and orientations used for this experiment were different than those used for the deformation experiments to help determine whether camera location and orientation affected measurement accuracy. A stationary set of dots was also used as reference points for the calculation of the translation of the targets on the thin-film mirror. Figure 6-12 presents the complete set up for the first translation experiment, where the stationary points can be seen on the white strip in the form of an $L$. Proper care was taken to ensure that the white strip did not touch the mirror during translation to prevent unintended motion of the reference points. Figure 6-13 shows one of the

photographs of the first translation experiment in which the fabricated pattern, marked dots and the reference points are seen. 


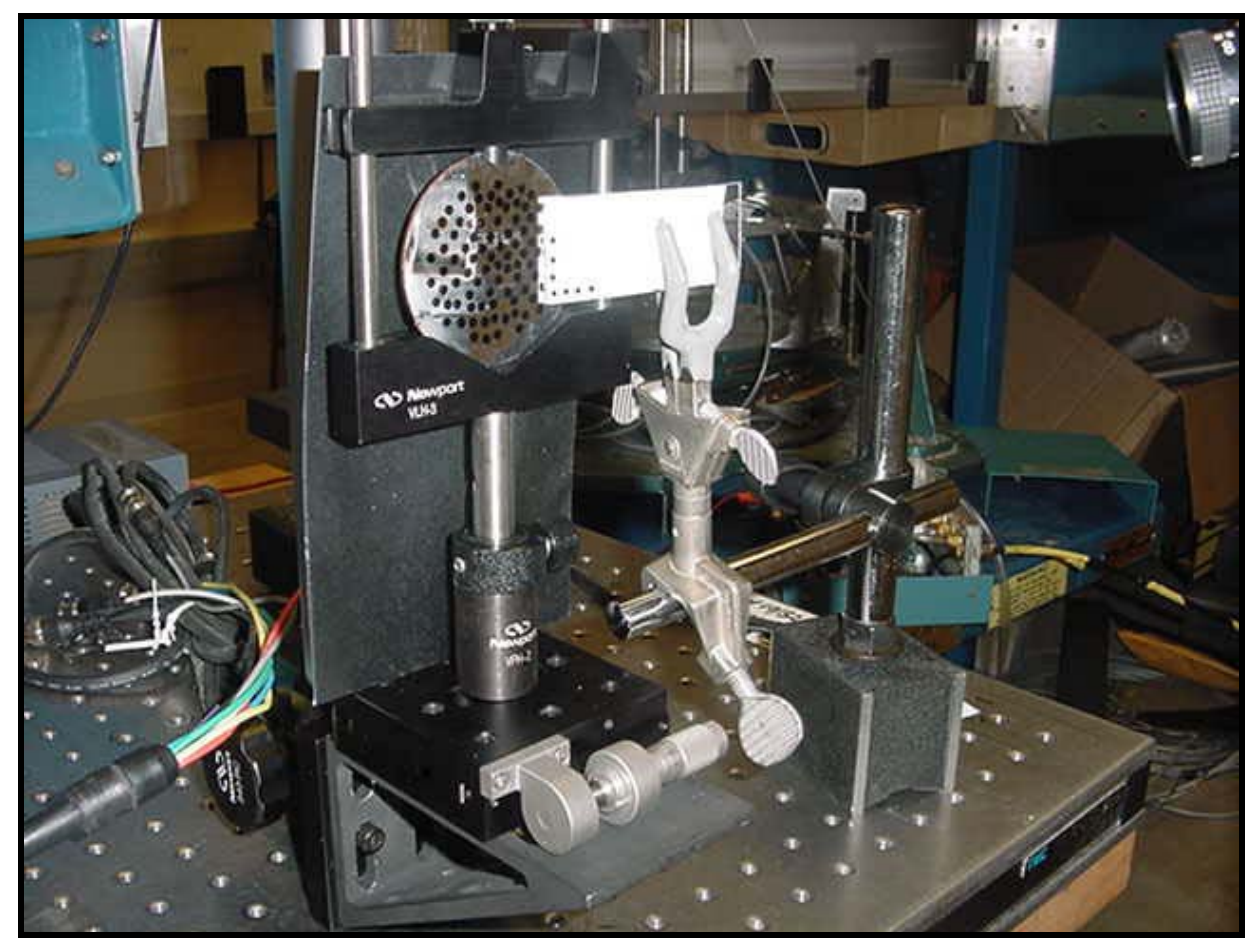

Figure 6-12: Experimental setup for the first translation experiment.

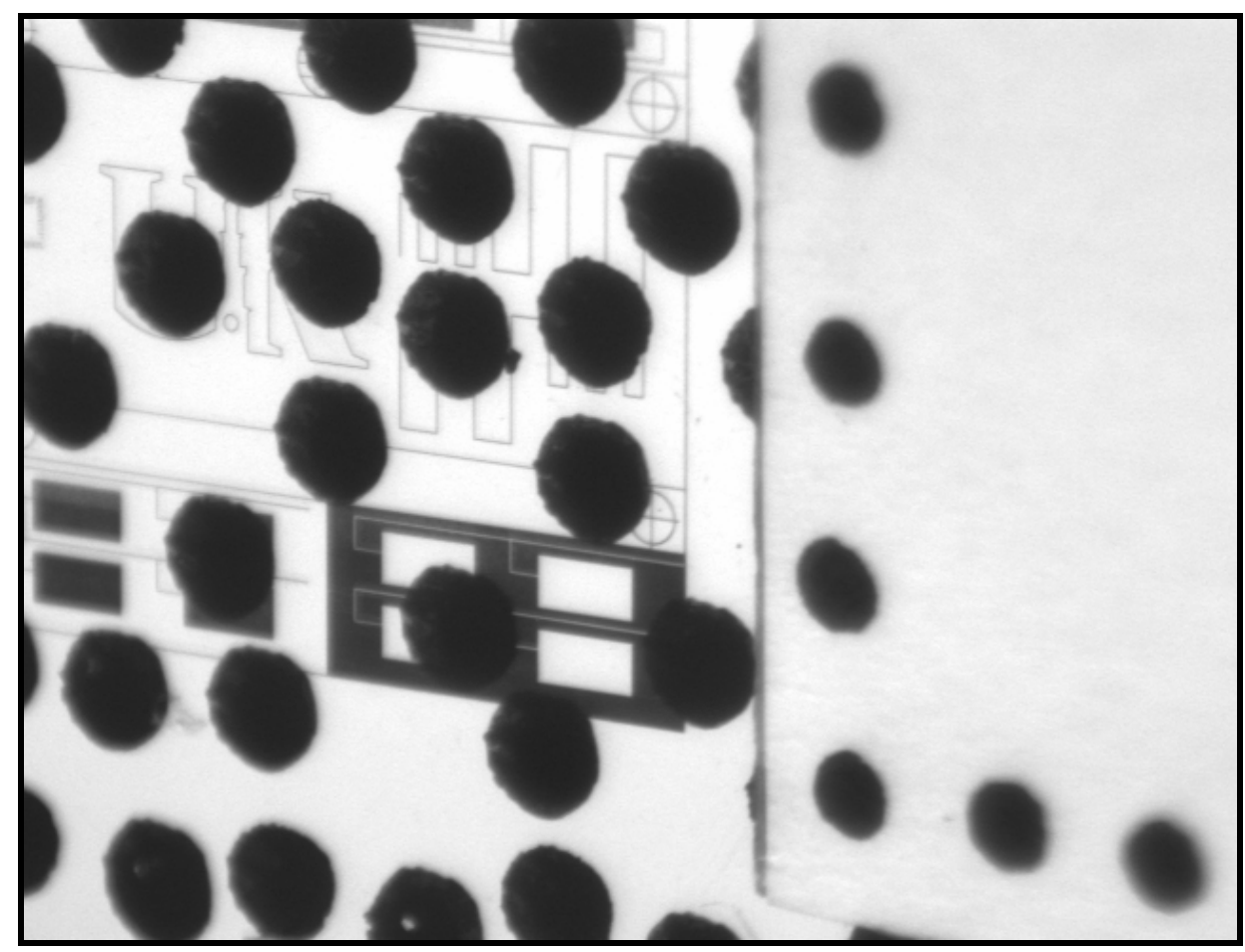

Figure 6-13: Photograph of the thin-film mirror taken in the first translation experiment. Both dots and fabricated pattern were used as targets. 
The experimental setup for the second and third translation experiments was same as the first translation experiment. A smaller translation step size of $0.001 \mathrm{~cm}$ was used for these two experiments. Smaller-size dots were also used on the thin-film mirror along with the larger dots and the fabricated pattern as targets. Smaller-sized dots were also used as reference points. The second translation experiment was performed to study the effect of the dot-size on the accuracy of measurement and to compare sub-pixel target marking and manual target marking. For the second experiment the camera was moved to four different locations for each translation step.

For the third translation experiment, initially the camera was fixed at one location and the translation increments were given from 0 to $0.005 \mathrm{~cm}$ in steps of $0.001 \mathrm{~cm}$. Then the camera was moved to the second location and all the translation steps were repeated from 0 to $0.005 \mathrm{~cm}$. In this fashion photographs were taken for all the translation steps from four fixed camera locations. This was done to examine error due to movement of the camera to the four locations for each translation step. This experiment provides insight into the future dynamic tests because the motion of the thin-film mirror will be captured from different cameras and divided into time sequence of images. Hence the third translation experiment replicates in some ways the dynamic tests except that only one camera was used to capture the motion instead of multiple cameras. Figure 6-14 shows one of the photographs of the third translation experiment. The photographs of the second translation experiment are similar to Figure 6-14. 


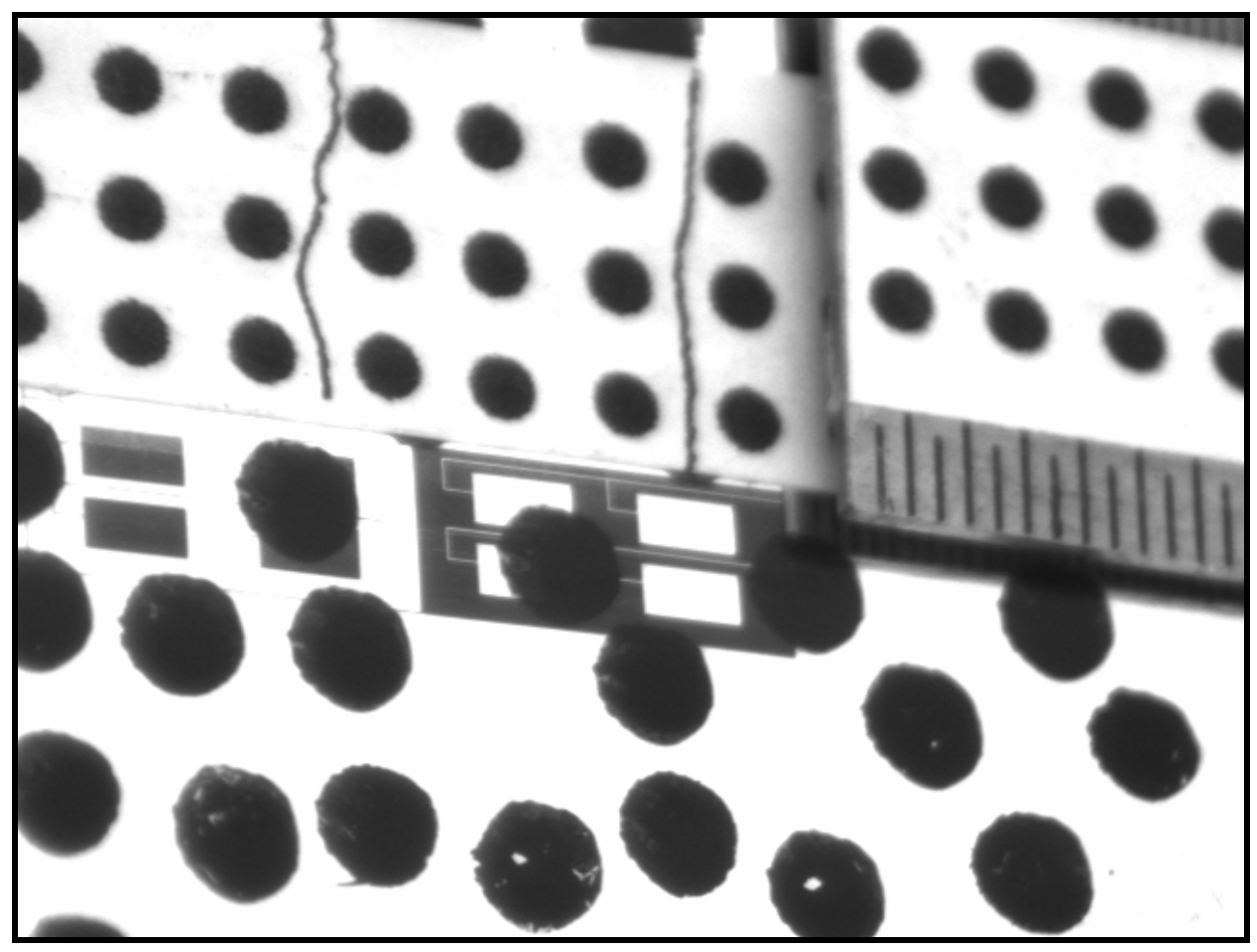

Figure 6-14: Photograph of the thin-film mirror taken in the third translation experiment

\subsubsection{Results of the Translation Experiments}

Figure 6-15 shows the results of the first translation experiment. Displacement of the linear stage is plotted on the horizontal axis and displacement calculated using PhotoModeler Pro v4.0 is plotted on the vertical axis. It is evident from the graph that the displacement calculated using PhotoModeler is accurate. This is because the ground sample distance of the camera, which is $0.005125 \mathrm{~cm}$, is orders of magnitude smaller than the translated distance of $1 \mathrm{~mm}$.

Figure 6-16 shows the results of the second translation experiment, plotted as before. Sub-pixel target marking was performed for smaller as well as larger circles and manual target marking was done for one point. The response of each of the targets is plotted separately. Though the sub-pixel target marking shows an increasing trend, the values of the displacement are higher than the input displacement given via linear stage. The manually marked target gives the largest variation and no evident trend. Displacements calculated using smaller dots are more accurate and show a regularly increasing trend, whereas the larger dot is more irregular. 


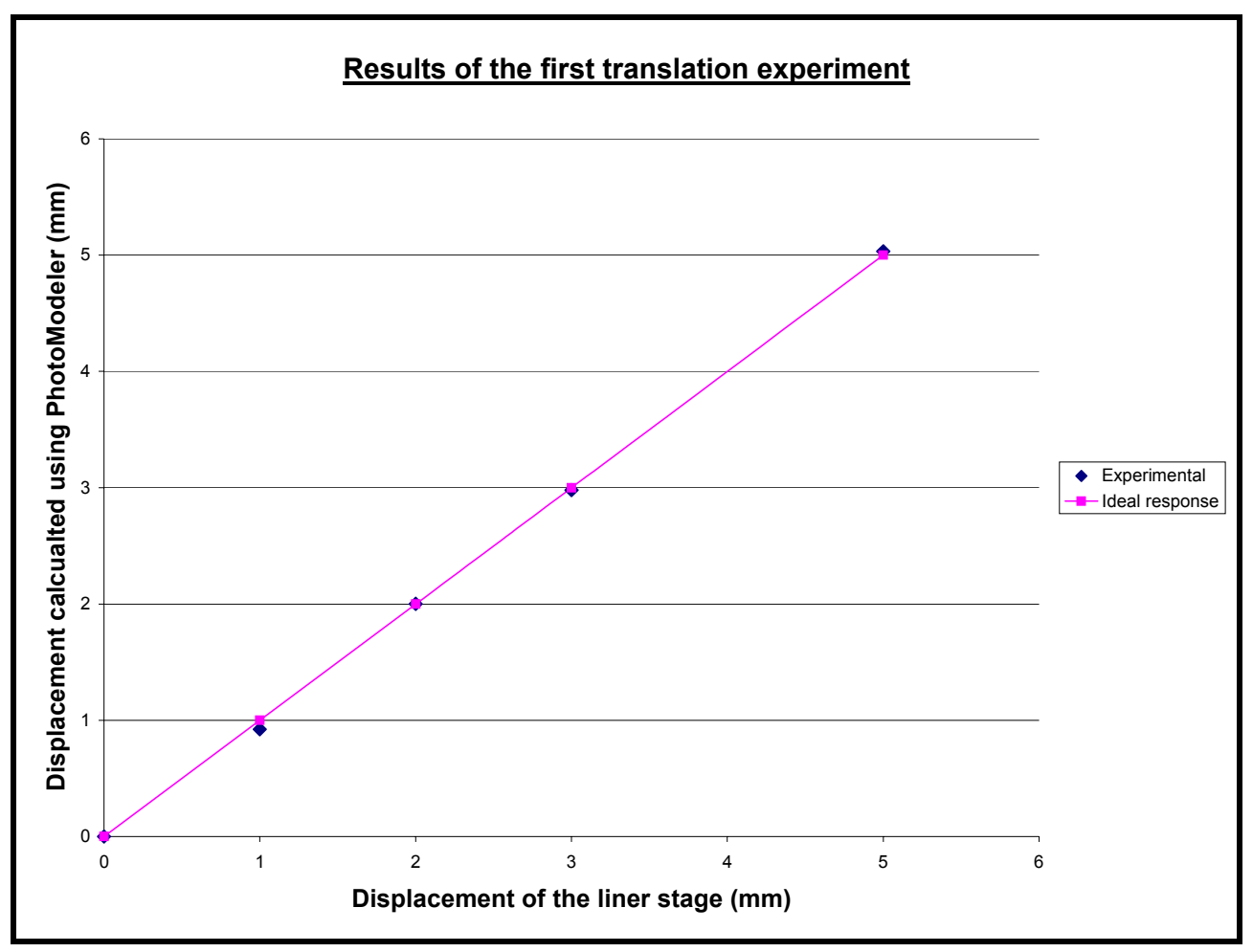

Figure 6-15: Results of the first translation experiment

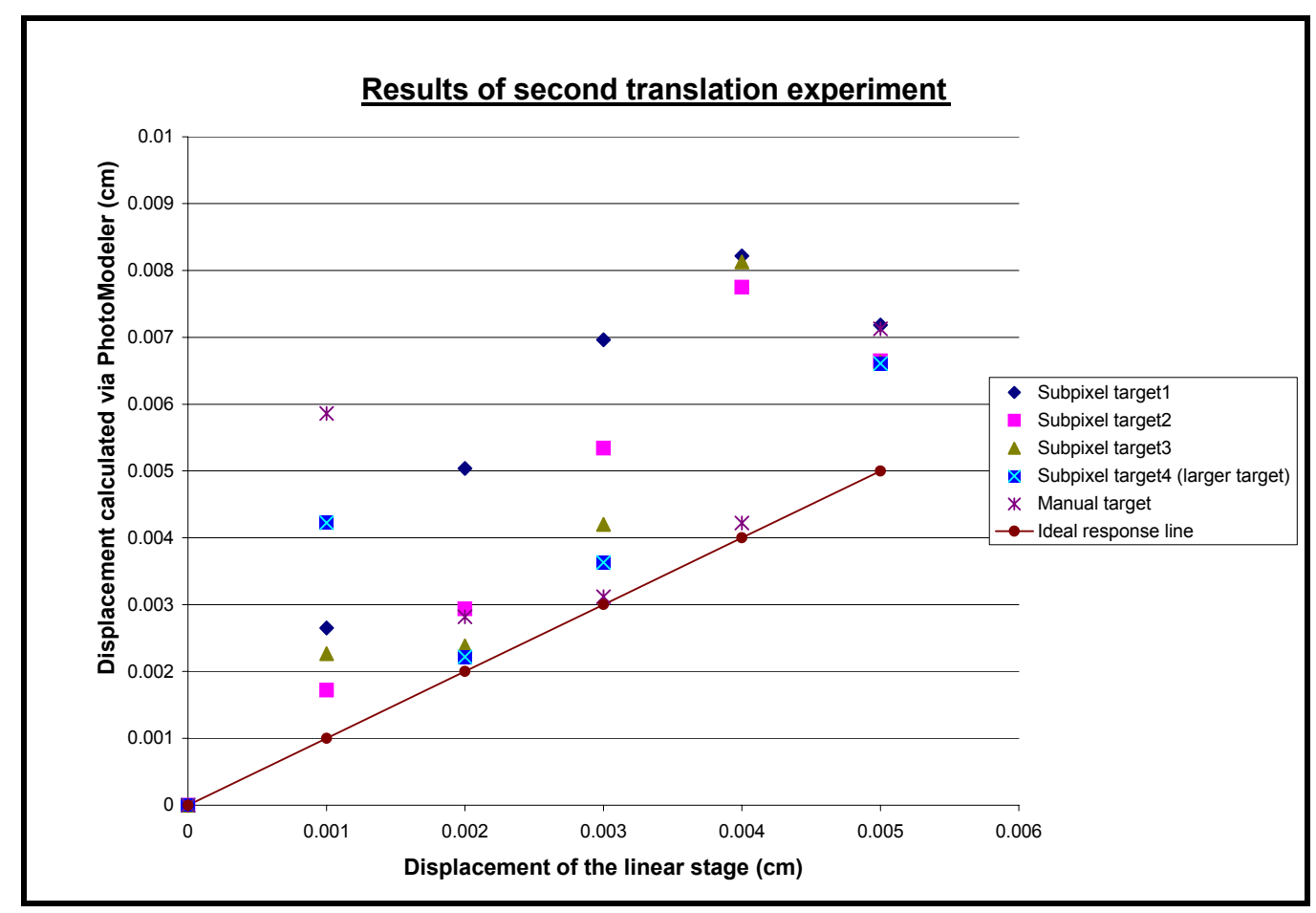

Figure 6-16: Results of the second translation experiment 
Figure 6-17 shows the results of the third translation experiment. Here again manual target marking is most irregular. Sub-pixel marking of smaller targets is seen as indicating the increasing trend with the least irregularity. None of these provide $0.001-\mathrm{cm}$ accuracy, though. With this procedure, identified results are both greater than and less than the input translation.

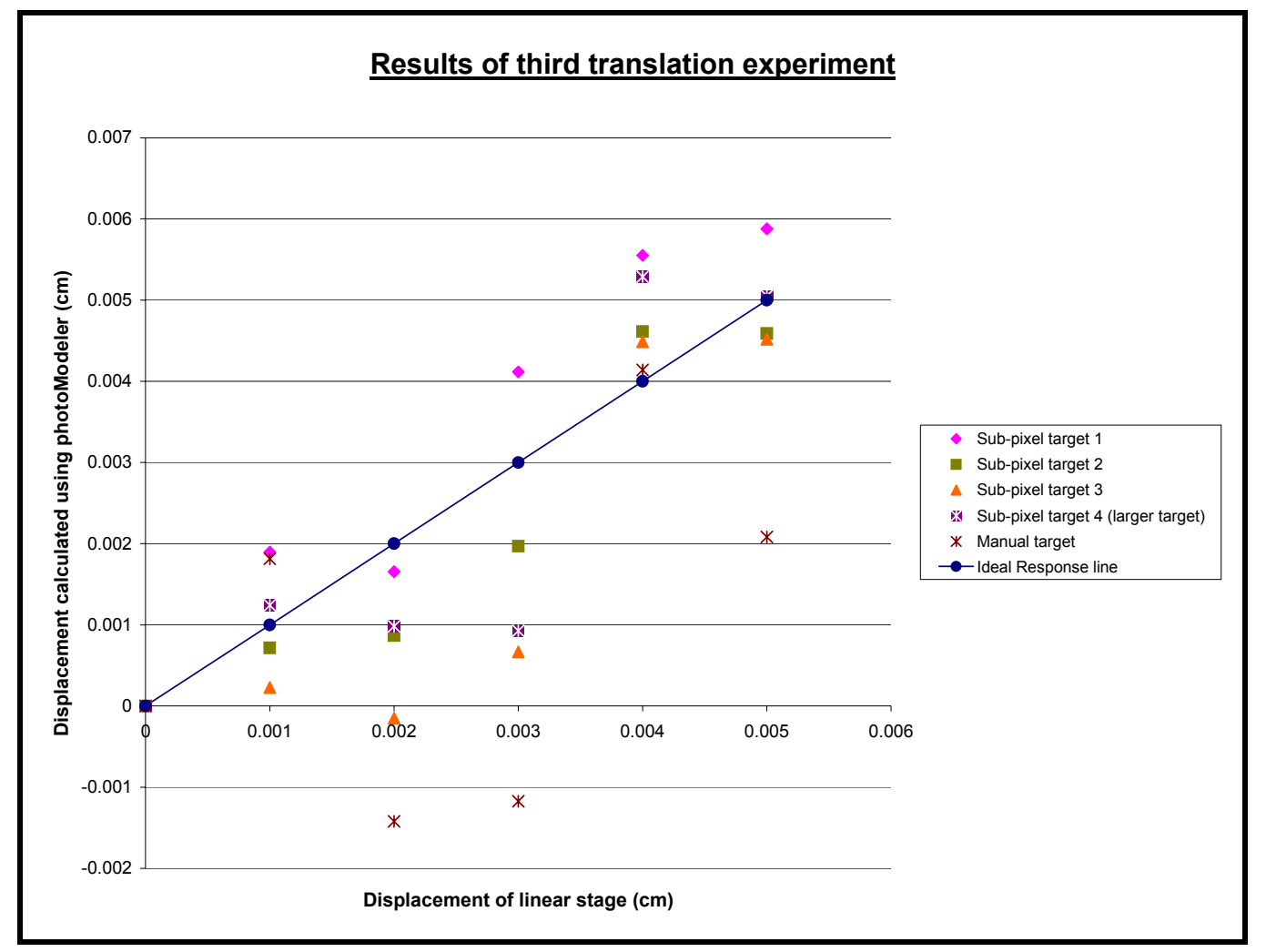

Figure 6-17: Results of the third translation experiment

\subsection{Preliminary Dynamic Experiment}

Figure 6-18 presents two frames of a very preliminary dynamic experiment with the patterned test article. Motion of the thin film was excited by blowing on it. A digital video camera (Pulnix TM - 6701 AN) was used to film the resulting motion. Individual frames showed deformation patterns resembling first and second mode shapes. Two frames with shadows indicating motion similar to that expected for the second mode pair are seen in Figure 6-18. Attempts to measure the fundamental frequency of the test article failed since the available laser displacement sensor requires a nonreflective surface for accurate measurement. 


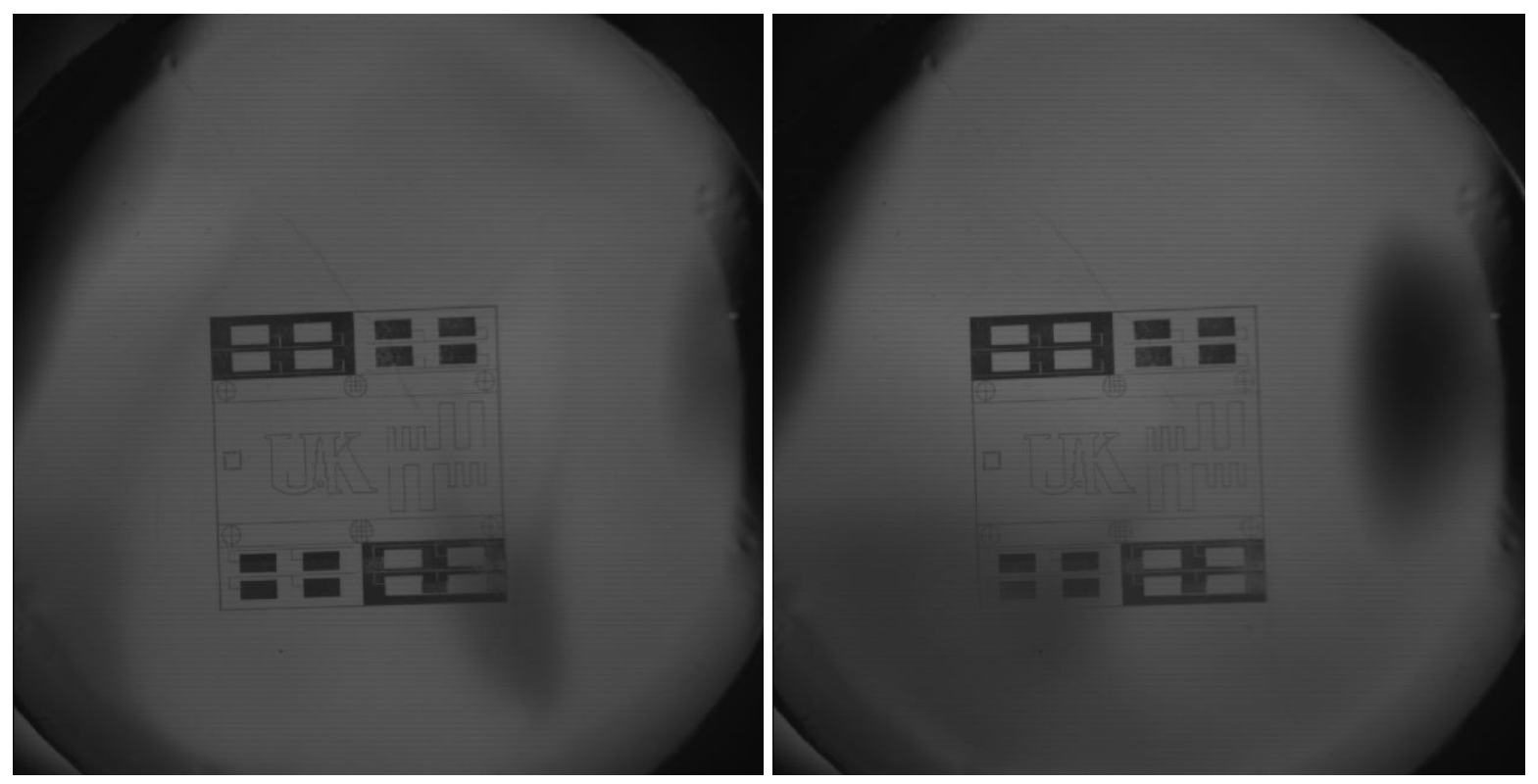

Figure 6-18: Frames showing deformation patterns resembling second mode

Copyright (C) Phanikrishna Thota 2003 


\section{Chapter 7: SUMMARY, CONCLUSIONS AND FUTURE WORK}

\subsection{Introduction}

Much new information was obtained from the research presented in this thesis pertaining to the measurement of in-plane displacement of thin films. Thin-film mirrors were manufactured and a pattern consisting of several features was fabricated using a Krypton Fluoride ( $\mathrm{KrF})$ excimer laser system. Atmospheric affects on the surface of the mirrors were studied during the course of fabrication. Finite element analysis was preformed on the thin-film mirrors to predict their natural frequencies, mode shapes and displacements under static load. Ultra close-range photogrammetry, using PhotoModeler Pro Vv4.0, measured both in-plane and out-of-plane displacements of the thin-film mirrors. Static experiments were conducted to calibrate the photogrammetric system for future dynamic tests. Different sets of photographs were recorded with application of force to the test article and also by giving pure translation in the plane of the mirror to the platform on which the sample was staged. An evaluation of accuracy of the measurements resulted.

\subsection{Summary and Conclusions}

This section is organized according to the objectives defined at the beginning of the thesis. The objectives are restated, along with results and the conclusions drawn from those results.

\section{Define an optimal surface pattern that has minimal influence on the dynamic response} of the thin films.

- Features used for regular tracking such as cross hairs, horizontal and vertical lines, serpentine patterns and features used for radar applications, were included in the pattern to be fabricated.

- A Finite element model of a 0.5-m diameter thin-film mirror was created. A grid pattern was modeled in an arbitrarily chosen 1 square inch area and the metallization in this area was removed in 40 steps in the form of grid. Modal analysis was performed for each of the 40 stages. As the non-metallization increased in the 1 square inch area, the first elastic 
mode pair shifted from their initial values but not significantly. The mode shape did not change with increase in non-metallization but the nodal diameter about which the mode shape is symmetric shifted to align with the fabricated patern so as to make the motion of the structure symmetric.

- Four finite element models similar to proposed test articles were created: two of them had the fabricated pattern while two of the models had the copper support ring. Modal analysis of the four finite element models was performed and the fundamental frequencies were compared to study the effect of the pattern and the copper ring. The test article modal analysis conclusion is that the fabricated pattern in the form of the nonmetallization does not significantly affect the dynamics of the thin-film mirror.

\section{$>$ Evaluate fabrication process for surface patterns.}

- Fifteen thin-film mirrors were fabricated on copper rings for support. Five of each available material were fabricated: single-sided aluminum coated on polyester, doublesided aluminum coated on polyester and single-sided gold-coated polyimide. Control of humidity is required to preserve mirror flatness. The thin-film mirrors were placed in an airtight container with desiccant packets in it to absorb the moisture in the container and to retain the mirror looking surface of the thin-film mirrors.

- A Krypton Fluoride $(\mathrm{KrF})$ excimer laser was used to fabricate pattern features on the thin-film mirrors. A parameter study was conducted to obtain the optimum values for the parameters, laser energy, chamber pressure, aperture size and beam attenuation. Slightly less energy was required to fabricate a pattern on the gold-coated polyimide thin-film mirror. Note that optimum values for the parameters changed when the laser gas cylinders were refilled. 


\section{Establish the basis for a precision and robust measurement technology for in-plane motion of thin-films structures based on ultra close-range photogrammetry.}

- Two experiments were performed on the thin-film test articles: 1) applying a force at the center of the mirror and 2) translating in the plane of the mirror.

- Without fixed reference points in the photographs, displacements cannot be determined. Fixed reference points in the form of cross wires were provided for the first experiment. Fixed reference points in the form of a stationary pattern of dots were provided for the second experiment.

- 3D co-ordinates of targets points were obtained for all force steps of the first experiment. Of interest were 14 different lines on the surface of the mirror. The normalized change in line length with increase in force calculated via experiments was compared with the finite element analysis. Discrepancy in the results from the finite element analysis and photogrammetry was attributed to two factors:

1. Material non-linearities were not included in the finite-element models, so permanent deformation in the yielded experimental test article was not modeled.

2. The resolution of the camera used to take pictures of the statically deformed test article was insufficient to capture the displacements, since the ground sample distance calculated from photographs was $0.005125 \mathrm{~cm}$. A camera with a 5 times higher resolution would give sufficient results.

- Pure translation was given to the mirror in known quantities in the plane of the mirror surface for the second experiment. Three translation experiments with different conditions were performed varying displacement step size, target size, camera mounting process, and target marking approaches.

- The accuracy of the measurement can also be improved. Multiple cameras and optimumsize areas for sub-pixel marking also improve accuracy.

In summary, the pattern fabricated on the thin-film mirrors does not have significant effect on its dynamics. Thin film can be mounted on a copper ring for support and Krypton Fluoride $(\mathrm{KrF})$ excimer laser system can be used to fabricate the desired pattern on to the thinfilm mirror. The method of photogrammetry can be effectively used to calculate the in-plane 
displacement using high-resolution cameras, correct camera locations and orientations and subpixel target marking. Experimental results can be used to verify computational models.

\subsection{Recommendations for Future work}

Material non-linearities can be included into the finite element models and further comparison made between them and the experimental results will help in the verification of the computational models. Optimum size features have to be fabricated onto a larger thin-film mirror using the excimer laser system so that sub-pixel marking can be performed to make the dynamic test analysis automated.

Copyright (C) Phanikrishna Thota 2003 


\section{BIBLIOGRAPHY}

1. Crawley, E.F., J.L. Sigler, M.C. Van Schoor, and M.J. Gronet, "Hybrid Scaled Structural Dynamic Models and Their Use in Damping Prediction." J Guidance Control and Dynamics, Vol. 13, No. 6, Nov-Dec 1990, pp. 1023-1032.

2. Javeed, M., H.H. Edighoffer, and P.E. McGowan, "Correlation of Ground Tests and Analysis of a Dynamically Scaled Space Station Model Configuration,” NASA TM 107729, 1993.

3. Campbell, J.E., S.W. Smith, and J.A. Main, "Staged Microgravity Deployment of a Pressurizing Scale Model Spacecraft.” AIAA Gossamer Forum, Denver, CO, April 2002

4. ESA-P. Nielsen, Plastics for Space-Problem Solvers on Earth http://www.esa.int/export/esaCP/ESA7SZZK0TC_index_1.html

5. The website for the space industry, International Space Station, International http://www.space-technology.com/projects/space_station/space_station6.html

6. Jenkins, C.H.M. (editor), "Gossamer spacecraft: Membrane and Inflatable Structures Technology for Space Applications ", Vol. 191, Progress in Astronautics and Aeronautics.

7. Freeland, R.E., Bilyeu, G., and Veal, G.R., "Validation of a Unique Concept for a Low-Cost, Lightweight Space Deployable Antenna Structure," Acta Astronautica, Vol.35, No. 9-11, 1995, pp. 565-572.

8. Veal, G., and Freeland, R., "IN-STEP Inflatable Antenna Description,” AIAA Paper 95-3739, Sept. 1995.

9. Satter, C.M., and Freeland, R.F., "Inflatable Structures Technology Applications and Requirements," AIAA Paper 95-3737, Sept.1995.

10. Freeland, R.E., Bilyeu, G.D., and Veal, G.R., "Development of Flight Hardware for a Large, Inflatable-Deployable Antenna Experiment," International Astronautical Federation, paper 951,5.0.1. Oct. 1995.

11. Freeland, R.E., Bilyeu, G.D., and Veal, G.R., "Development of Flight Hardware for a Large, Inflatable-Deployable Antenna Experiment," Acta Astronautica. Vol.38, Nos. 4-8 1996, pp 351260. 
12. Post, D., "Moire Interferometry: Advances and Applications," Experimental Mechanics, November 1991, pp. 276-280.

13. One system for all coherent optical NDT, Projected Fringe Moiré www.pcholo.com/opticals.htm

14. Research webpage of Gianni "Nick" Nicolette, Professor, Dipartimento di Ingegneria Industriale, Universita degli Studi di Prma, Italy http://me.unipr.it/design/nick/research .html.

15. Martinez, A., Rodriguez-Vera, R., Rayas, J. A., Puga, H. J., "Fracture Detection by Grating Moiré and In-plane ESPI Techniques". Optics and Lasers in Engineering 39 (2003) 525-536.

16. Hansen, R.S., "A compact ESPI system for displacement measurements of specular reflecting or optical rough surfaces," Optics and Lasers in Engineering, In Press.

17. Singh, M., and G. Ramachandran, "Reconstruction of Sequential Cardiac In-plane Displacement Patterns on the Chest wall by Laser Speckle Interferometry," IEEE Transactions on Biomedical Engineering, Vol.38, No.5, May 1991, pp. 483-489.

18. Sarrafzadeh-Khoee, A. and J.C. Duke, Jr., "Small In-plane/Out-Of-Plane Ultrasonic Displacement Measurement using Laser -Speckle Interferometry," Experimental Techniques, October 1986, pp. 18-21.

19. Tong, J., D-S Zang, H-Q Li, and L-N Li, "Study on In-Plane Displacement Measurement under Impact Loading using Digital Laser-Speckle Pattern Interferometry.” Optical Engineering, Vol. 35. No. 4, April 1996, pp 1080-1083.

20. Mazza E., Danuser G., and Dual J. 1996. Light optical deformation measurements in microbars with nanometer resolution. Microsystem Technologies 2(2): 83 - 91.

21. Toigo, A., P. J. Gierasch, and M. D. Smith, High Resolution Cloud Feature Tracking on Venus by Galileo, Icarus, 109, pp. 318-336, 1994 “

22. Tracker - Image-processing and Object-Tracking System Developed http://www.grc.nasa.gov/other_Groups/RT1998/6000/6729klimek.html .

23. Campbell, J.E., S.W. Smith, and J.A. Main, "Staged Microgravity Deployment of a Pressurizing Scale Model Spacecraft.” AIAA Gossamer Forum, Denver, CO, April 2002 
24. Shi Jianbo and Tomasi Carlo, "Good Features to Track", IEEE Conference on Computer Vision and Pattern Recognition (CVPR) Seattle, June 1994.

25. Rembe, C., Kant, R., Muller, R.S., Berkeley Sensor \& Actuator Center, University of California Berkeley, "Optical Measurement Methods to Study Dynamic Behavior in MEMS". http://www-sac.eecs.berkeley.edu/projects/memstesting/SPIEPaper4400_15.pdf

26. Timothy Schmidt and John Tyson, Trilion Quality Systems, Konstantin Galanulis, GOM mbH, “Dynamic Strain Measurement Using Advanced 3D Photogrammetry”, IMAC- XXI,2003. 27. Otto Frei, "Tensile Structures", Massachusetts Institute of Technology, Cambridge, MA, 1962.

28. Leonard, R.W., Brooks, G.W., and McComb, H.G., Jr., "Structural Considerations of Inflatable Reentry Vehicles", NASA Technical Note D-457, 1960.

29. Topping, A.D., "Shear Deflections and Buckling Characteristics of Inflated Members", Journal of Aircraft, 1(5), pp. 289-293, 1964.

30. Bulson, P.S., “Design Principles of Pneumatic Structures”, Structural Engineering, 51(6), pp. 209-215, 1973.

31. Douglas, W.J., "Bending Stiffness of an Inflated Cylindrical Cantilever Beam", AIAA Journal, 7(7), pp. 1248-1253, 1969.

32. Steeves, E.C., "A Linear Analysis of the Deformation of Pressure Stabilized Tubes", U.S. Army Natick Laboratories Report, AD/A-006 493, NTIS, N75-32513.

33. Fichter, W.B., “A Theory for Inflated Thin-Wall Cylindrical Beams”, NASA Technical Note D-3466, June 1966.

34. Comer, R.L., and Levy, S., "Deflections of an Inflated Circular-Cylinder Cantilever Beam”, AIAA Journal, 1(7), pp. 1652-1655, 1963.

35. Webber, J.P.H., "Deflections of Inflated Cylindrical Cantilever Beams Subjected to Bending and Torsion", Aeronautical Journal 86(858), pp. 306-312, 1982.

36. Main, J.A., Peterson, S.W., and Strauss, A.M., "Load-Deflection Behavior of Space-Based Inflatable Fabrics Beams”, Journal of Aerospace Engineering, 7(4), 1994. 
37. Main, J.A., Carlin, R.A., Garcia, E., Peterson, S.W., and Strauss, A.M., "Dynamic Analysis of Space-Based Inflated Beam Structures", Journal of the Acoustical Society of America, 97(2), pp. 1035-1045, Feb. 1995.

38. Slade, Kara N., and Tinker, Michael L., "Analytical and Experimental Investigation of the Dynamics of Polyimide Inflatable Cylinders", AIAA-99-1518, Proceedings of the 40th Structures, Structural Dynamics, and Materials Conference, April 12-15, 1999, St. Louis, MO.

39. Dharamsi, U. K., Evanchik, D. M., and Blandino, J. R., "Comparing Photogrammetry with a Conventional Displacement Measurement Technique on a Square Kapton Membrane ", AIAA paper 2002-1258, Proceedings of the 3rd AIAA Gossamer Spacecraft Forum, April 2002.

40. Jenkins, C.H., Haugen, F., and Spicher, W.H., "Experimental Measurement of Wrinkling in Membranes Undergoing Planar Deformation," Experimental Mechanics, Vol. 38, No. 2, June 1998, pp. 147-152.

41. Jenkins, C.H., Fitzgerald, D.M., Liu, X., “Wrinkling of an Inflatable Membrane with Thermo-Elastic Boundary Conditions, " 41 st AIAA Structures, Structural Dynamics, and Materials Conference, Atlanta, GA, Paper No. AIAA-2000-1727, April 2000.

42. Blandino, J.R., Johnston, J.D., Miles, J.J., Soplop, J.S., "Thin-Film Membrane Wrinkling due to Thermal and Mechanical Loads," $42^{\text {nd }}$ AIAA Structures, Structural Dynamics and Materials Conference, Seattle, WA, Paper No.AIAA-2001-1345, April 2001.

43. Black, J.T. and Pappa, R.S., "Videogrammetry Using Projected Circular Targets: Proof-ofConcept Test", Proceedings of the 21st International Modal Analysis Conference, February 2003.

44. Pappa, R.S., Black, J.T., Blandino, J.R., Jones, T.W., Danehy, P.M., Dorrington, A.A., "DotProjection Photogrammetry and Videogrammetry of Gossamer Space Structures", NASA/TM2003-212146, January 2003

45. Pronko PP. Dutta SK, Squier Jnm. Opt Commu, Rudd JV, Du D, Mourou G. "Machining of submicron holes using a femtosecond laser" at 800 n 1995; 114:106-10.

46. Liu X, Du D, Mourou G. "Laser ablation and micromachining with ultrashort laser pulses". IEEE J Quantum Electron 1997; 33(10): 1706-16. 
47. Liu X. "Submicron lines in thin metal films micromachined by an ultrafast laser oscillator". Technical digest -Conference on Lasers and Electro-Optics, 1998. P .511.

48. Bado P, Clark W, said AA. "Hand book of look micromachining", 1999. http://www.cmxr.com/micromachining/handbook.

49. Kautek W, KrÜger K. "Femtosecond pulse ablation of metallic, semiconducting, ceramic, and biological materials". Proc SPIE 1994; 2007:600-11.

50. Venkatakrishnan, K., Tan, B., Ngoi, B.K.A., "Femtosecond Pulsed Laser Ablation of Thin Gold Film”. Optics \& Laser Technology 34(2002) 199-202.

51. Pfleging. W., Ludwig, A., Seemann, K., Preu, R., Mäckel, H., Glunz, S.W., "Laser micromachining for applications in thin film technology", Applied Surface Science 154155(200) 633-639.

52. Rumbsy, P., Harvey, E., Thomas, D., N. Rizvi., SPIE 3184 (1997) 176.

53. Naessens, K., Heidi Ottevaere, P. Van Daele, Baets, R., "Flexible fabrication of microlenses in polymer layers with excimer laser ablation”, Applied Surface Science 9587(2003) 1-6.

54. T. Djenizian, L. Santinacci, P. Schmuki, App.Phys Lett.78(19) (2001) 2940.

55. T. Von Woedtke, P. Abel, J. Krüger, W. Kautek, Sens. Actuators 42(1997) 151.

56. M. Van Damme, J. Vermeersch, F. Louwet, F.Samijn, "Heat sensitive imaging element for making lithographic printing plates”, EP96-203130-08.10.97.

57. Y. Nakayama, S. Nishi, H. Ishibashi -Ueda, T. Matsuda, Biomed, Mater.Res. 51(2002) 520.

58. T. Matsuda, Y. Nakayama, Biomed. Mater. Res. 31 (1996) 235.

59. Callewaert, K., Martelé, Y., Breban, L., Naessens, K., Vandaele, P., Baets, R., Geuskens, G., Schacht, E., "Excimer laser induced patterning of polymeric surfaces", Applied Surface Science 9597(2003) 1-8.

60. Atkinson, K. B. (editor), Close Range Photogrammetry and Machine Vision, Whittles Publishing, Scotland, UK, 2001.

61. Shortis, M. R. and Beyer, H. A., Sensor Technology for Digital Photogrammetry and Machine Vision”, ibid., pp. 106-155. 
62. Pappa, R. S., Jones, T. W., Black, J. T., Walford, A., Robson, S., Shortis, M. R., "Photogrammetry Methodology Development for Gossamer Spacecraft Structures", AIAA-20021375.

63. SLAMA, C., ed 1960. "The manual of photographic Interpretation. Bethesda, MD American society fro photogrammetry and remote sensing".

64. SLAMA, C., ed 1980. "The manual of photographic Interpretation. Bethesda, MD American society fro photogrammetry and remote sensing".

65. Laussedat, Col. A., Recherches sur les instruments, les methods et le dessin topographiques, Paris, Gauthier- Villars, Vol.1 1898; Vol II, pt.1, 1901, pt. 2,1903.

66. Thompson, M.M., (editior), Eller, R.C., Radlinski, W.A., Speert, J.L.,(Associate editiors), "Manual of photogrammery", Third edition. Volume 1.

67. Hallert, B., "Photogrammetry- Basic principles and general survery”, McGraw-Hill Book Company, Inc. 1960.

68. Mikhail, E.M., Bethel, J.S., McGlone, J.C., “Introduction to modern photogrammetry” John Wiley \& Sons, Inc. ISBN 0-471-30924-9.

69. Rosato, D.V. and Schwartz, R.T., (Editors), "Environmental Effects on Polymeric Materials", 1968.

70. Gayatri Rallapalli, Master's Thesis, "Excimer Laser Assisted Fabrication of Diffractive Optics", 1997.

71. Hua Li, Master's Thesis, “Aluminum Metallization of Aluminum Nitride by KrF Excimer Laser Processing", 1996.

72. Leena Vakil, Master's Thesis, “Fabrication of Coplanar Waveguides Using Excimer Laser”, 1998.

73. David J. Elliott, “ Ultraviolet Laser Technology and Applications,” Academic Press, Inc., New York. (1995)

74. B.Braren, “ Materials Patterning with Excimer Laser Ablation”, Optics and Photonics News, Vol. 3, pp 20 - 23, 1992. 
75. ANSYS User's Manual for Revision 5.0 Volume 3, Elements. Swanson Analysis Systems, Inc.

76. Properties of $\mathrm{LaRC}^{\mathrm{TM}}-\mathrm{CP} 1$ and $\mathrm{LaRC}^{\mathrm{TM}}-\mathrm{CP} 2$, SRS Technologies, Huntsville, Alabama. http://www.stg.srs.com/polyimidesales/filmproperties.htm

77. MatWeb, Material property data, Aluminum alloys, General

http://www.matweb.com/search/SpecificMaterial.asp?bassnum=MA0001

78. Mikhail, E.M., J.S. Betherl, and J.C. McGlone, Introduction to Modern Photogrammetry, John Wiley \& Sons, New York, NY, 2001.

79. Photomodeler Pro - User Manual. Eos systems Inc, Vancouver, BC, Canada.

80. Shortis, M. R. and Beyer, H. A. - Sensor technology for close range photogrammetry and machine vision. In: Close Range Photogrammetry and Machine Vision, K. B. Atkinson (Editor).Whittles Publishing, Caithness, pp. 106-155.

81. L' Garde, Inc., "Photogrammetry Capability,"

http://www.lgarde.com/capabilities/photo.html.

82. Giersch, L.R., Pathfinder Photogrammetry Research for Ultra-Lightweight and Inflatable Space Structures, M.S. Thesis, George Washington University, Aug. 2001 (also published as NASA CR-2001-211244, Nov. 2001)

83. SONY Component / OEM,http://www.sony.net/Products/ISP/pdf/catalog/XC55E.pdf 


\section{VITA}

I was born in Nizamabad, India on April 24, 1980 and lived there for seventeen years until I moved to Hyderabad to attend the Jawaharlal Nehru Technological University College of Engineering in August 1997 for my Bachelors. I was awarded two gold medals and a state sponsored scholarship for my academic achievement in high school along with a Junior Chamber award for topping the district. I was also a part of the volleyball team, which won the state championship in 1995. I won the Best Student Award in 1995 and 1997 as a consequence of my all-round performance in both secondary and high school. I received my Bachelors degree in Mechanical Engineering in June 2001.

I came to the United States of America to attend the University of Kentucky in July 2001. I joined the Masters program at the University of Kentucky with Mechanical Engineering as my major. I joined Dr. Suzanne Weaver Smith and Dr. Jack Leifer because of my long-lasting fascination for space-structure dynamics. I worked on a freshly started project helping my advisors to put forward a proposal with my initial research, which was granted funds later. I was involved in writing a conference paper "Initial Pattern Evaluation for In-plane displacement Measurement of Thin Films", co-authored with Dr. Suzanne Weaver Smith, Dr. Jack Leifer and Dr. Janet K. Lumpp for IMAC-XXI, February 2003, at Kissimmee, Florida. I was awarded "Outstanding Graduate Student" for my scholarship by the College of Engineering for the year 2003. I am at the verge of submitting a journal paper for "Experimental Mechanics".

Phanikrishna Thota 